\title{
The Alphatrap experiment ${ }^{\star}$
}

Sven Sturm ${ }^{\mathrm{a}}$, Ioanna Arapoglou, Alexander Egl, Martin Höcker, Sandro Kraemer, Tim Sailer, Bingsheng Tu, Andreas Weigel, Robert Wolf ${ }^{b}$, José Crespo López-Urrutia, and Klaus Blaum

Max-Planck-Institut für Kernphysik, Saupfercheckweg 1, 69117 Heidelberg, Germany

Received 11 December 2018

Published online 11 February 2019

\begin{abstract}
The Alphatrap experiment at the Max-Planck Institute for Nuclear Physics in Heidelberg aims at probing the validity of quantum electrodynamics in extremely strong electromagnetic fields. To this end, Alphatrap will determine the value of the magnetic moment, or the $g$-factor, of the electron bound in highly charged ions. Quantum electrodynamics predicts this value with extraordinary precision. As the bound electron in highly charged ions is exposed to the strongest fields available for high-precision spectroscopy in the laboratory, reaching up to $10^{16} \mathrm{~V} / \mathrm{cm}$ in hydrogenlike lead ${ }^{208} \mathrm{~Pb}^{81+}$, a comparison of the theoretical prediction with a measured value can yield the most stringent test of the Standard Model in strong fields. The targeted precision of eleven digits or more can be achieved by storing single highly charged ions in a cryogenic Penning trap, where its eigenfrequencies can be determined with ultra-sensitive electronics to highest precision. Additionally, the spin state can be non-destructively determined using the continuous Stern-Gerlach effect, allowing spectroscopy of the Larmor precession. Alphatrap is constructed to enable the injection and the storage of externally produced ions. The coupling to the Heidelberg EBIT gives access to even the heaviest highly charged ions and thus extends the available field strength by more than two orders of magnitude compared to previous experiments. This article describes the technical architecture and the performance of ALPHATRAP and summarises the experimental measurement possibilities.
\end{abstract}

\section{Introduction}

The Standard Model (SM) of physics describes the interactions of particles via the four fundamental forces - electromagnetic, weak and strong interaction as well as gravitation. The former three interactions are represented by quantum field theories, whereas gravitation up to now has resisted attempts to be treated in such a framework. Quantum electrodynamics (QED), the interaction of electrically charged particles via the exchange of photons, has been the first of these quantum field theories

* This article comprises parts of the thesis work of I.A., A.E. T.S. and A.W.

a e-mail: Sven.Sturm@mpi-hd.mpg.de

b Present address: ARC Centre of Excellence for Engineered Quantum Systems, School of Physics, The University of Sydney, NSW, Australia. 
and served as a blueprint for the development of the other theories. Accordingly, the experimental verification of this theory is of major importance and interest. Indeed, QED is today considered to be the best tested theory of the SM. The magnetic moment associated with the spin of the electron can be calculated within the framework of QED and has thus served for experimental tests of the theory. Probably the most intriguing of these experiments is the $g-2$ experiment of the free electron [1], which has determined the gyromagnetic ratio, or $g$-factor, of the electron up to the 13th decimal. This unique agreement of the experiment with the prediction by the SM is a far-reaching confirmation of the validity of the theory. However, in this experiment, except for the weak magnetic trapping field, the electron couples only to the vacuum field, so that non-linear effects in the field strength might go unnoticed.

In order to specifically address such interactions, for example a hypothetical photon self-interaction or any other strong-field deviation of the known interactions, tests at the strongest possible electromagnetic field are desirable. Externally applied static fields are limited in strength to about $50 \mathrm{~T}$ for static or $300 \mathrm{~T}$ for pulsed magnetic fields, or to about $1600 \mathrm{kV} / \mathrm{cm}$ in vacuum gaps [2] in case of electrostatic fields. These limitations can be overcome in intense laser fields. However, typically the local intensity in the laser beam is transient in time and space, as well as only poorly controllable, hindering high-precision experiments.

By binding a single electron to a heavy nucleus, this electron is exposed to its extremely strong Coulomb field. Expectation values reach up to about $10^{16} \mathrm{~V} / \mathrm{cm}$ in the ground state of such hydrogen like heavy ions. Such field strengths are many orders of magnitude higher than achievable in current state-of-the-art laser fields. The properties of these systems are inherently well defined. QED allows for an extraordinarily precise calculation of electronic transition frequencies in few-electron ions. The strong binding reduces the effect of external perturbations, such as electromagnetic fields originating from the trap. Simultaneously, transition frequencies from the electronic ground state to an excited state scale strongly with the nuclear charge $Z$. Orbital transition energies increase with $Z^{2}$, transitions between hyperfine-structure levels with $Z^{3}$ and fine-structure transitions with $Z^{4}[3,4]$. These scalings make most orbital transitions in heavy highly charged ions (HCI) inaccessible for optical lasers, which hinders high-precision experiments. Despite this, a series of experiments in the experimental storage ring (ESR) at the GSI Helmholtz Center for Heavy Ion Research have successfully determined the ground-state Lamb shift of HCI up to hydrogenlike uranium [5]. Here, the relevant transitions are well within the X-ray regime, such that a resonant excitation is not feasible. The experiment measures the energy of photons from spontaneous decays of excited ions. The Lamb shift, which amounts to only $460.2 \mathrm{eV}$, can be extracted from the difference of two transition frequencies [4].

A particularly suitable observable for a strong field test of the SM is the magnetic moment of the bound electron in heavy HCI. The ultra-precise measurement of this quantity, which is the main goal of ALPHATRAP, and the comparison with the prediction of the SM enables a stringent test of the validity of the SM in the yet largely unexplored regime of extremely strong electromagnetic fields. While the presence of the strong binding field of the nucleus largely influences the magnetic moment, its absolute value does not change critically over the complete chart of nuclides. Over the past years the $g$-factor experiment for HCI at the Johannes Gutenberg-University in Mainz, Germany has performed a series of measurements on different elements and charge states with 11 digits precision. The $g$-factor determination in hydrogenlike silicon ${ }^{28} \mathrm{Si}^{13+}[6,7]$ has provided the most stringent test of QED in strong fields to date. By measuring suitable combinations, such as the isotopic difference of the $g$-factors of lithiumlike ${ }^{40,48} \mathrm{Ca}^{17+}[8]$, it was possible to test the relativistic recoil effect on the nucleus. Finally, an ultra-precise measurement of the $g$-factor of ${ }^{12} \mathrm{C}^{5+}$ allowed to determine the value of the atomic mass of the electron, a fundamental constant of the SM, with world-leading precision [9]. Eventually, the Mainz experiment was 
hindered by the achievable ionisation energies from measuring even heavier systems and thus to test QED in extremely strong fields. Here, AlPHATRAP's key advantage lies in the possibility of injecting ions from external ion sources, such as the Heidelberg EBIT [10]. In combination with the versatile spin-state detection via the continuous Stern-Gerlach effect (CSGE, see Sect. 3.6) Alphatrap will be able to explore an extensive range of interesting measurement candidates. In absence of an effective nuclear spin, the theoretical prediction is not influenced by the distribution of the magnetic moment inside the nucleus, the Bohr-Weisskopf effect (BWE). Although the finite size of the nucleus does indeed alter the Coulomb interaction, the theoretical prediction is significantly more precise than in the case of the hyperfine structure. In fact, by measuring the magnetic moment in different charge states of one isotope, it becomes possible to largely cancel the influence of the nucleus and thus to compare the measurement with the most precise quantum-electrodynamical calculations [11]. This will yield a unique opportunity to perform the most sensitive test of the validity of the SM in extremely strong electromagnetic fields.

While in general most electronic ground-state transitions are inaccessible for optical lasers in heavy HCI, in specific systems there are exceptions to this rule. The fine structure (FS) and hyperfine structure (HFS) of the ground state, which leads to a small splitting of the spectroscopic lines in the range of microwave frequencies for hydrogen, evolves into the regime of optical frequencies for medium heavy or heavy ions, respectively. This opens the possibility to perform laser spectroscopy of the HFS in these ions. The magnetic field produced by the nuclear magnetic moment at the position of the electron can reach values up to $10000 \mathrm{~T}$, making these tests especially interesting. However, since the HFS is sensitive specifically to small distances, it is also strongly influenced by the spatial distribution of the spin inside the nucleus, which leads to the BWE. Since there is only very limited knowledge of this distribution, the BWE causes a severe limitation on the precision of the theoretical prediction. By making use of the dependence of the BWE on the electronic state, it is possible to eliminate the BWE from the measurement by considering specific differences of the hyperfine splittings in different charge-states. Recently, the LIBELLE collaboration measured the HFS in lithiumlike and hydrogenlike bismuth ions in the ESR storage ring $[12,13]$. Both systems have an unpaired electron in an s-state, such that their overlap with the nucleus is similar up to a scaling factor. By using literature values for the magnetic moment of the bare nucleus, it is possible to predict the weighted difference of the HFS in these systems to a relative precision of about $10 \mathrm{ppm}$. The experimental result differed from that prediction by seven standard deviations at the time of the publication, raising the question of the validity of the Bound-State Quantum Electrodynamics (BS-QED) calculations in these heavy systems. After the publication, a nuclear magnetic resonance measurement [14] yielded a new value for the nuclear magnetic moment of bismuth, which recovered consistency with theory, however at the cost of a larger uncertainty. This yields a further strong motivation to not only further investigate the HFS in heavy HCI with higher precision, but also to perform direct measurements of the nuclear magnetic moments, which would eliminate the major source of uncertainty from the BS-QED test. Only a very limited number of systems are accessible to such measurements, as the transitions in both charge-states need to be in the optical regime. In the bismuth system, the long life-time of the transitions severely complicates the measurement. In Section 6.3 we propose a novel method to measure FS and HFS transitions in HCI. With this technique, the Alphatrap experiment will address FS and HFS transitions in heavy HCI and independently determine nuclear magnetic moments with high accuracy. Finally, as will be detailed in Section 6.5, it might eventually become possible to extract a value of the electromagnetic fine-structure constant $\alpha$ with AlPHATRAP by making use of the dependence of the $g$-factor on relativistic wave functions in the bound state (see Sect. 2.1). An independent value with competitive 
precision is an important contribution for other precision tests of the SM with atomic systems.

\section{Theory}

\subsection{The $g$-factor of a bound electron}

After the seminal experiments by Stern and Gerlach [15] and their interpretation by Uhlenbeck [16] it became clear that the electron has an intrinsic angular moment, the so-called spin, which is associated with a magnetic moment $\mu_{s}$. With the formulation of his relativistic quantum theory in 1928 [17], Paul Dirac not only explained the observed two-fold separation in the Stern-Gerlach experiment, but also enabled the calculation of the strength of the magnetic moment in units of the Bohr magneton $\mu_{\mathrm{B}}$ :

$$
\mu_{s}=-g_{s} \mu_{\mathrm{B}} \frac{\vec{s}}{\hbar}
$$

The $g$-factor, which appears as the unitless proportionality constant, has served as a work-horse along the development of the SM. Since it can be measured with extraordinary precision, a comparison with the theoretical prediction immediately yields a stringent test of the validity of the theory. The Dirac theory predicts the $g$-factor of the electron propagating freely in space to be exactly $g=2$. As the $g$-factor describes the interaction of the electron's magnetic moment with a magnetic field, it appears as parameter for observables in atomic physics, such as the hydrogen spectrum with its HFS. Only with the high-precision measurements by Kusch and Foley [18] it became apparent that $g_{s}$ deviates slightly but significantly from Dirac's prediction. This discovery led to the development of modern QED, which allows for the emission and absorption of real and virtual photons by the electron. The leading-order terms (see Fig. 1) have been calculated by Schwinger [19], yielding $g_{s}=2\left(1+\frac{\alpha}{2 \pi}\right)$, where $\alpha \equiv \frac{e^{2}}{4 \pi \epsilon_{0} \hbar c} \approx \frac{1}{137}$ is the fine-structure constant, which parametrises the coupling strength of the electron to photons. Here, $\epsilon_{0}$ is the vacuum permittivity and $c$ the speed of light in vacuum.

Since this advent of QED, the calculations have been dramatically refined. Today, the $g$-factor of a free electron, or equivalently the anomaly $a_{e} \equiv \frac{g_{s}-2}{2}$, is calculated in a QED series expansion:

$$
a_{e}=\sum_{n=1}^{\infty} C_{2 n}\left(\frac{\alpha}{\pi}\right)^{2 n} .
$$

Using this terminology, the Schwinger term $C_{2}=\frac{1}{2}$. Today, the coefficients up to $C_{10}$ have been calculated [20,21], yielding more than sufficient precision to compare with the experimental value of $g_{s}-2$, which has been measured in 2008 at Harvard university in the Gabrielse group [1]. In order to use this result to test QED, an independent determination of the fine-structure constant is required. On the current level of precision, this can be obtained from experiments that measure the photon recoil momentum on atoms. A recent experiment on cesium atoms [22] was able to determine $\alpha$ to 0.2 parts-per-billion (ppb) precision. By inserting this value into the QED series expansion and comparing with the $g_{s}-2$ experiment results in a $2.5 \sigma$ tension. Though this is clearly not enough to claim a discovery, it might be interpreted as a hint to new physics. 


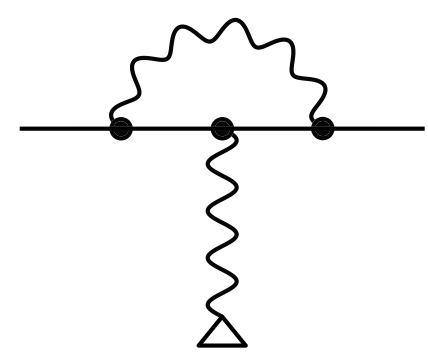

Fig. 1. Feynman diagram for the one photon-loop correction for the free electron (Schwinger term). Prior to the interaction with the external magnetic field, denoted here by a triangle, the electron emits a virtual photon, which it reabsorbs later in order to conserve total energy. This next-to-leading order contribution changes the $g$-factor by about one permille relatively.

When the electron is not propagating freely, but is bound in the Coulomb potential of a hydrogen like ion, it is no longer described by a plane wave solution in the Dirac equation but rather by the hydrogen wave functions. Accordingly, also the $g$-factor (in the $1 s$ state) is no longer $g=2$ even in lowest order. Breit was the first to calculate the solution to the Dirac equation [23], which was named after him:

$$
g_{1 s}=2 \frac{1+2 \sqrt{1-(Z \alpha)^{2}}}{3} .
$$

While $g_{1 s}$ is similar to the free space solution for light ions, in the heavy ion regime, where the nuclear charge $Z$ is such that $Z \alpha \approx 1$, the dependence on $\alpha$ becomes sizeable. For hydrogenlike lead the deviation amounts to about $13 \%$ and a measurement of $g_{1 s}$ can potentially be used to determine the precise value of the fine-structure constant. Additionally to this purely relativistic effect, also the QED contributions change compared to the free electron case. The main goal of ALPHATRAP is to explore whether QED stays valid in such strong fields by comparing experimental measurements with predictions by QED (the field strength experienced by a $1 s$ electron is plotted in Fig. 2). The corresponding BS-QED theory can be calculated in two fundamentally different ways: Either the QED interactions with the magnetic field are evaluated for a freely propagating particle and the interaction with the Coulomb potential is accounted for by separate QED interactions, leading to a second series expansion in orders of $Z \alpha$. Alternatively all Feynman diagrams are evaluated using the hydrogen solutions in the QED propagator, yielding a solution in all orders of $Z \alpha$. In the low- $Z$ regime, where the Coulomb field is relatively weak, the $Z \alpha$ expansion converges fast and can yield extremely precise values. In the high- $Z$ regime however, where the field is extremely strong and $Z \alpha \approx 1$, the QED interactions with the Coulomb field become strongly coupled and a series expansion does not converge satisfyingly anymore. This strong coupling opens the possibility to probe a new regime of physics in extremely strong fields. To exploit this possibility it is mandatory to calculate all relevant Feynman diagrams, which are summarised and grouped in the following.

\subsection{Radiative corrections}

The interactions with the magnetic field are structured according to the number of photon loops. As every photon loop effectively contributes a factor of $\alpha$, higher loop 


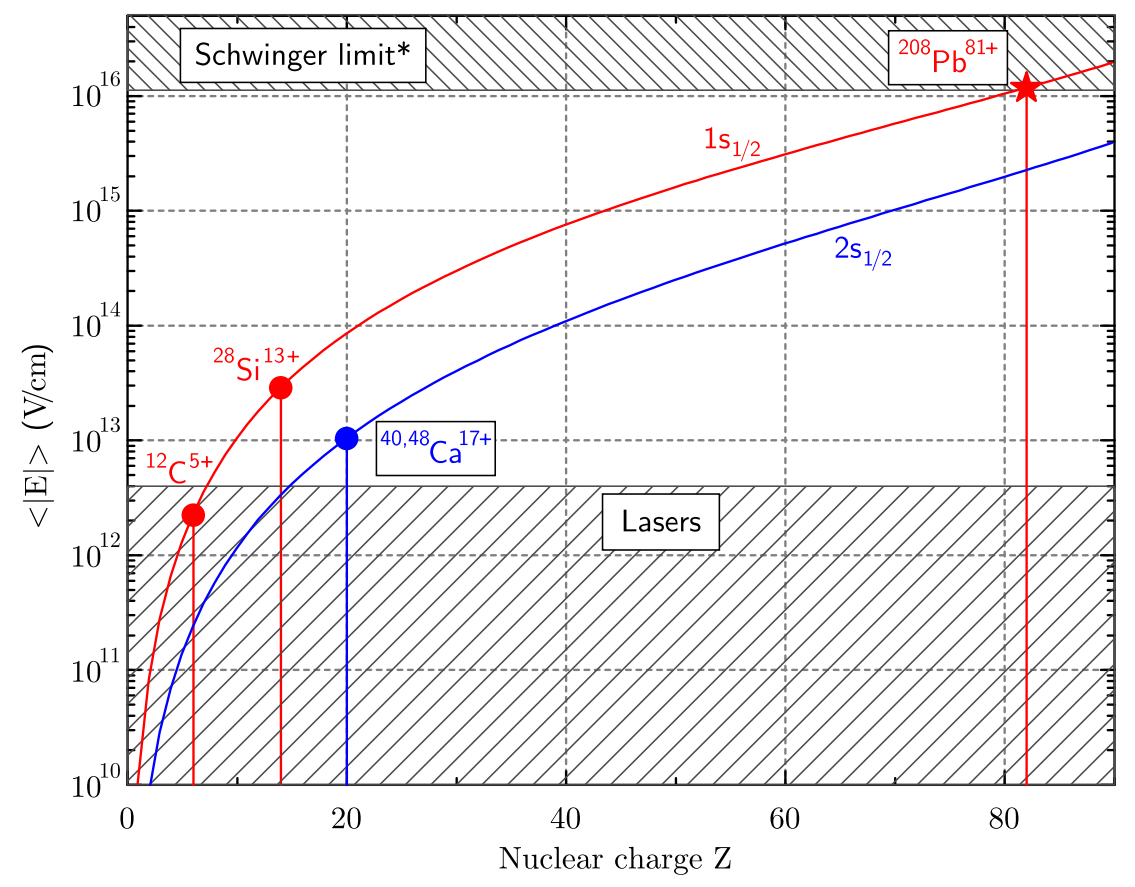

Fig. 2. Relativistic expectation value $\left\langle\left|E_{n s}\right|\right\rangle$ of the electric field strength in the $1 s$ (hydrogenlike) and $2 s$ (lithiumlike) states. $\left\langle\left|E_{1 s}\right|\right\rangle$ surpasses the values achievable in the brightest laser sources today already in the medium- $Z$ regime. In the high- $Z$ regime the bound electron is exposed to the strongest field available in the laboratory for precision experiments on stable systems. Systems that have been measured already at the Mainz HCI experiment and their respective field strength regime are displayed for reference, as well as hydrogenlike lead ${ }^{208} \mathrm{~Pb}^{81+}$, the heaviest system to be targeted by Alphatrap. $\left(^{*}\right)$ The Schwinger limit shown here corresponds to the homogenous field case, the microscopic volume of the ion prevents the spontaneous breakup of the vacuum by creation of electron-positron pairs in the range of nuclear charges displayed here.

orders contribute accordingly less. The six diagrams of the first loop order, which are shown in Figure 4, are calculated to all orders of $Z \alpha$ within the Furry picture [25], where the nucleus is accounted for by a fixed, stationary Coulomb potential. In second order, there are already 50 Feynman diagrams. At present these diagrams have not yet been evaluated in all orders of $Z \alpha$ but in an expansion up to $(Z \alpha)^{5}$. When combined with the higher order diagrams of unbound QED (order $\alpha^{n}(Z \alpha)^{0}$ [21]) these results are extremely precise in the low- $Z$ regime and thus allow not only for tests of QED but also to extract fundamental constants from the respective measurements (see Sect. 6.2). However, in order to also reach a similar precision in the high- $Z$ regime it is mandatory to derive results in all orders of $Z \alpha$, a large effort which is currently being pursued in several groups worldwide.

\subsection{Contributions from the nucleus}

In reality, the binding potential, which is originating from the nucleus, is not a true Coulomb potential. Instead, the finite size and internal structure of the nucleus causes significant deviations from a pure Coulomb potential close to the nucleus. When making use of charge radii which have been measured in independent experiments it 


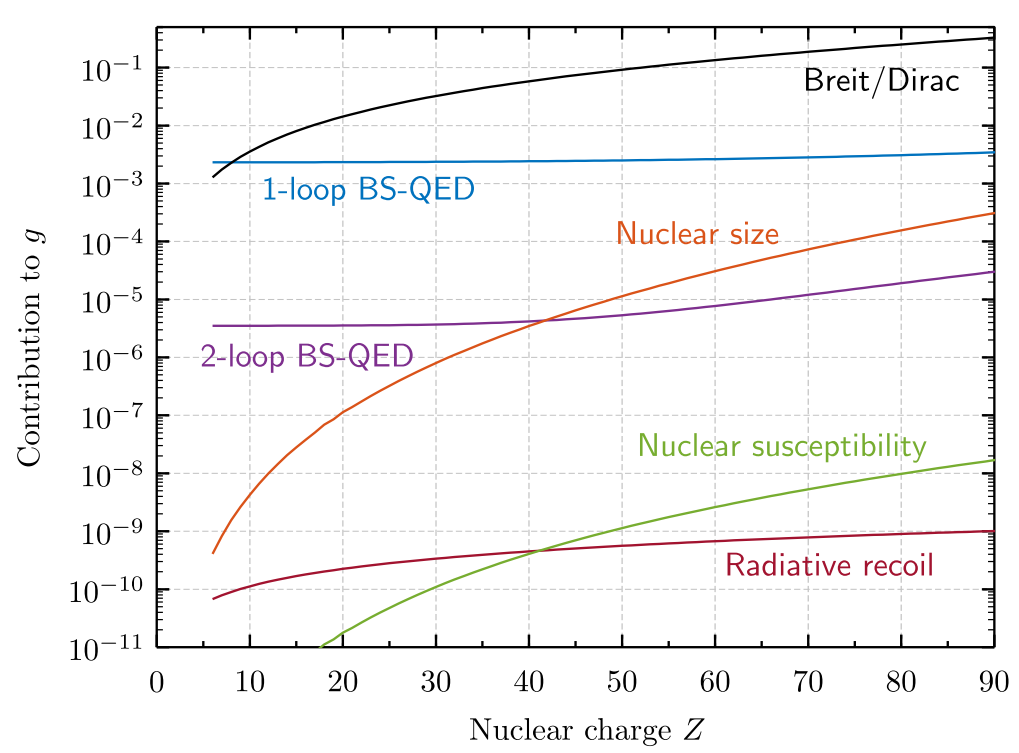

Fig. 3. Contributions to the $g$-factor of the bound electron in hydrogenlike ions. The projected relative experimental precision of the ALPHATRAP experiment is about 10 parts-per-trillion (ppt) and thus enables an exquisite test of BS-QED in the strongest electromagnetic fields. It can be seen that most contributions increase in size with the nuclear charge. Especially the contributions of the nuclear charge distribution (finite size) and nuclear structure (susceptibility) become sizeable in the heaviest elements, but even there, compared to other observables such as the HFS, the interesting QED contributions are clearly resolvable and can be stringently tested [24].

becomes possible to allow for the more accurate potential shape in the calculations and derive respective corrections. These correction are called the finite size effect. For small nuclei also the finite size effect is relatively small and can be accounted for to very good precision. For heavy ions however, the finite size effect is approaching $0.1 \%$ in the heaviest systems (see Fig. 3) and the available literature values of the charge radius is implying severe limits on the achievable precision of the theoretical calculations [26]. On even higher precision also effects beyond the charge radius, originating from nuclear structure and susceptibility become relevant [27]. While the dependence of the $g$-factor on these effects can be used for determining very precise values of nuclear structure parameters from the experiments, in order to perform the most stringent QED test it is possible to cancel the impact of the nuclear effects by making use of the specific difference of $g$-factors in different charge states (see Sect. 6.1).

Additionally to the effect of the finite nuclear size, also the mass of the nucleus is not infinitely large. Accordingly, the interaction of the electron with the nucleus leads to a recoil of the nucleus which alters the observed $g$-factor [28]. Interestingly, while for electronic transitions the recoil effect can be calculated in good approximation in the reduced mass system where the center of mass of nucleus and electron stands still, only relativistic recoil effects contribute to the $g$-factor. As a result, the $g$-factor requires a non-trivial solution to the Dirac equation with two motile particles and thus provides an immediate access to physics beyond the Furry picture [8]. By comparing the $g$-factor of the same element and charge state but in different isotopes, most electronic QED contributions drop out and thus need not be calculated to all orders. This way, the isotopic effect allows us to specifically test the interesting yet small contributions of the nucleus. 


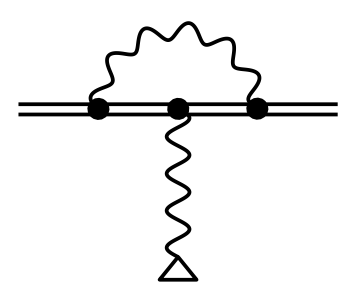

(a)

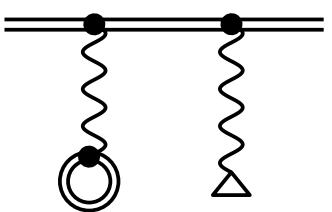

(d)

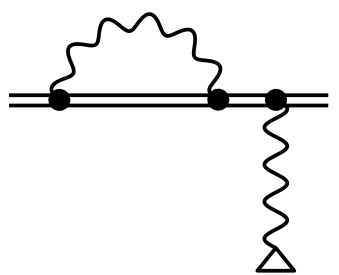

(b)

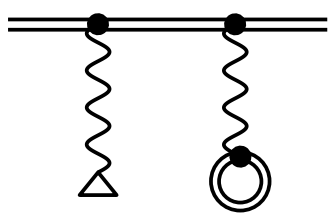

(e)

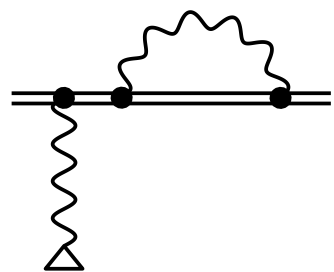

(c)

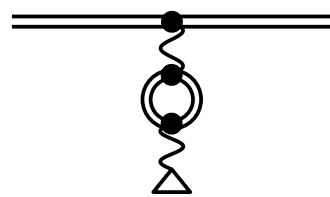

(f)

Fig. 4. The six Feynman diagrams of first order in the bound-state framework. Here, the double line indicates the bound-state propagator. Instead of considering the electron to be "free" in between interaction vertices, the Coulomb potential of the nucleus is accounted for by using the hydrogen solution of the Dirac equation as the basis for the propagator. Correspondingly, the diagram can be calculated in all orders of $Z \alpha$ and is thus applicable even to the heaviest HCI. (a) Corresponds to the Schwinger term, while the remaining diagrams represent self-energy and vacuum-polarization corrections to the $g$-factor, which are for the free electron not appearing explicitly but are accounted for by the renormalizations.

\subsection{Many-electron effects}

When considering systems with more than a single electron, also interactions between the individual electrons will contribute to the $g$-factor, which adds significant complexity. In Figure 5 a choice of Feynman diagrams is shown. It is possible to distinguish between diagrams that involve only photon exchanges in between electrons, which are summarised as interelectronic interactions, and those diagrams that involve closed photon loops in addition to the interelectronic photon exchange, which are called screened QED. Specifically the interelectronic interactions are interesting since they necessarily involve the negative energy states in the calculation. At present, the uncertainty of the interelectronic contributions dominate the uncertainty budget in the low- and medium- $Z$ regime. For the medium- to high- $Z$ regime Volotka et al. have recently provided rigorous QED solutions in all orders of $Z \alpha$ that significantly improved the precision compared to previous results $[29,30]$. For boronlike systems, the situation is even more complex due to the additional electrons. Here, similar rigourous calculations are still pending.

\section{Experimental principle}

Alphatrap is an ultra-high precision Penning-trap experiment dedicated to the measurement of ground-state properties of highly charged ions. A pivotal element is the detection of the spin-state of a single HCI via the continuous Stern-Gerlach effect, which was developed by Dehmelt et al. around the 1980s [31]. When combined with highly sensitive image current detectors, it becomes possible to determine the 


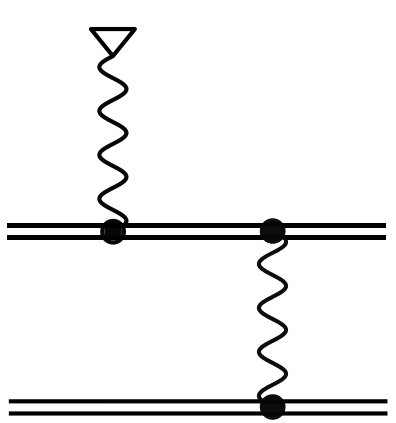

(a)

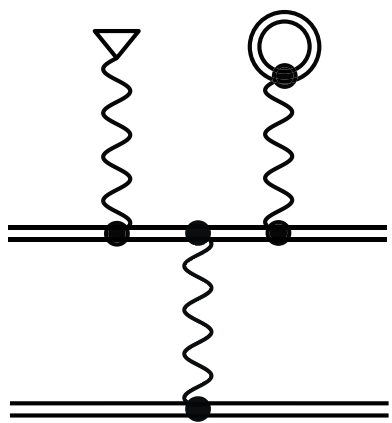

(b)

Fig. 5. Examples for Feynman diagrams in a few-electron system. (a) Represents interelectronic interaction, while (b) is assigned to the screened-QED contributions, where the QED interaction (here the vacuum polarization) of the active electron is altered by the presence of a second electron.

Larmor spin-precession frequency ${ }^{1}$

$$
\nu_{\mathrm{L}}=\frac{1}{2 \pi} \frac{g}{2} \frac{e}{m_{e}} B_{0},
$$

which depends on the electron's charge $e$, mass $m_{e}$ and the sought after gyromagnetic " $g$-factor", as well as the cyclotron frequency

$$
\nu_{\mathrm{c}}=\frac{1}{2 \pi} \frac{q}{m} B_{0}
$$

of the ion with mass $m$ and charge $q$ in the magnetic field $B_{0}$ of the Penning trap. From these two frequencies, it is possible to determine the $g$-factor of the HCI:

$$
g=2 \frac{\nu_{\mathrm{L}}}{\nu_{\mathrm{c}}} \frac{m_{e}}{m} \frac{q}{e}
$$

which then can be compared to theoretical predictions. Today, state-of-the-art QED calculations consider two-loop contributions and even three-loop diagrams are starting to become relevant $[11,32,33]$.

In the following, the techniques and challenges that are relevant for ALPHATRAP and its goal to ultimately achieve measurement precisions in the 10 parts-per-trillion regime and beyond are described.

\subsection{The Penning trap}

The Penning trap is the central tool for Alphatrap. Over the last decades, the Penning trap has proven its worth for the precise determination of a multitude of ground-state properties of charged particles. It consists of a superposition of a homogeneous magnetic field $\vec{B}=B_{0} \overrightarrow{e_{z}}$, which confines the particle in the plane

\footnotetext{
${ }^{1}$ In this work, we use frequency units for measurement observables wherever possible. In some cases however, the equivalent angular frequencies $\omega_{i}=2 \pi \nu_{i}$ with identical indices are used to prevent the appearance of $2 \pi$ factors.
} 
perpendicular to the field lines ("radial") due to the magnetic Lorentz force. A harmonic electrostatic potential prevents loss of the ion along the field lines ("axial"). The mathematically idealised trapping potential

$$
V_{\text {ideal }}(\rho, z)=V_{0} \frac{C_{2}}{2 d}\left(z^{2}-\rho^{2} / 2\right)
$$

which is characterised by a characteristic trap "length" $d$ and a field parameter $C_{2}$, would be produced from infinitely large, hyperbolically shaped electrodes. In highprecision Penning traps, this ideal potential is carefully approximated by a set of electrodes, which are in the case of AlPhATRAP of cylindrical shape. In such traps, the potential around the trap origin can be expressed in terms of a series expansion:

$$
V_{\text {real }}(\rho, \theta, z)=\frac{V_{0}}{2 d} \sum_{n=0}^{\infty} C_{n} \rho^{n} P_{n}(\cos \theta),
$$

where $P_{n}$ denotes the Legendre polynomials. The axial frequency $\nu_{z}$ for ions with negligible mode energies is given by the term of second order in $z$, which coincides with $V_{\text {ideal }}$ :

$$
\nu_{z}=\frac{1}{2 \pi} \sqrt{\frac{q V_{0} C_{2}}{m d^{2}}} .
$$

The even terms in equation (8) lead to typically unwanted shifts of $\nu_{z}$ as a function of the mode energies. A suitable geometric design of the trap electrodes, including socalled correction electrodes [34], allows nulling the dominant terms, typically at least $C_{4}$ and $C_{6}$. Such a trap is called compensated [35]. In the case of the Alphatrap trap, even higher orders up to $C_{10}$ are nulled. The odd terms are typically very weak due to the axial symmetry of the trap and are neglected because in leading order they produce no secular effect on the eigenfrequencies. While the magnetic field does not influence the axial motion, in the plane perpendicular to the magnetic field the trajectory of the ion is controlled by both the magnetic and the electric field. The homogeneous magnetic field alone would force the ion on cyclotron orbits with the free-space cyclotron frequency $\nu_{c}$. Combined with the electrostatic quadrupole field this is modified to a system of two independent harmonic eigenmotions: the fast modified cyclotron motion with frequency $\nu_{+}$and the slow magnetron drift around the electrostatic center of the trap with frequency $\nu_{-}$. These frequencies are related to the free-space cyclotron frequency $\nu_{c}$ via

$$
\nu_{ \pm}=\frac{\nu_{c}}{2} \pm \frac{1}{2} \sqrt{\nu_{c}^{2}-2 \nu_{z}^{2}}
$$

All three trap eigenfrequencies obey a typical hierarchy:

$$
\nu_{-}<\nu_{z} \ll \nu_{+}<\nu_{\mathrm{c}}
$$

As its name suggests, $\nu_{+}$is mainly defined by the magnetic field and amounts to about $25 \mathrm{MHz}$ for highly charged ions in the case of ALPHATRAP with $B_{0} \approx 4.02 \mathrm{~T}$, while $\nu_{z}$ (for a given ion) depends solely on the electric field and is adjusted by suitable choice of voltages to about $650 \mathrm{kHz}$. The magnetron frequency $\nu_{-}$, which is to first approximation mass independent, amounts to about $10 \mathrm{kHz}$. Since the eigenmotions are uncoupled in an ideal trap, the corresponding mode energies are conserved and can be easily calculated from the sum of kinetic and potential energy associated with 
the respective motion. The magnetron motion is special as it has relatively low kinetic energy due to the low frequency of the motion, and its energy is dominated by the negative potential energy:

$$
E_{-}=\frac{1}{2} m\left(\omega_{-}^{2}-\frac{\omega_{z}^{2}}{2}\right) \rho_{-}^{2} \approx-\frac{1}{4} m \omega_{z}^{2} \rho_{-}^{2}
$$

For the $g$-factor experiment, all three eigenfrequencies have to be measured. The determined values can then be used to calculate the free-space cyclotron frequency via the so-called invariance theorem [36,37]:

$$
\nu_{\mathrm{c}}=\sqrt{\nu_{+}^{2}+\nu_{z}^{2}+\nu_{-}^{2}} .
$$

Considering the hierarchy in equation (11), the individual frequencies have to be measured with different relative precisions. The highest precision is required for $\nu_{+}$. The invariance theorem (13) is robust against lowest-order imperfections of the trapping fields, especially a tilt of the magnetic and electric field axis and an elliptic distortion of the electrostatic field. Since such imperfections cannot be fully avoided even in carefully manufactured and aligned traps, this robustness is the key to performing ultra-high precision experiments in Penning traps.

\subsection{Image current detection}

For the $g$-factor measurements it is mandatory to detect the motion of the single ion non-destructively, i.e. without losing the ion. This can be achieved by a measurement of the ion's image current, see Figure 6 . The charged ion induces so-called mirror-charges into the conductive trap electrodes. When the ion oscillates with its eigenfrequencies between the electrodes, so do the mirror-charges. The current flowing in and out of the electrodes thus contains the desired frequency information of one or more of the eigenfrequencies, depending on the geometry of the electrodes. However, the small magnitude of this current, typically in the femtoampére regime, necessitates sophisticated cryogenic and superconducting electronics, which is detailed in Section 4.6. The induced charge on one trap electrode is given to first order by

$$
q_{\text {ind }}=q \frac{z}{D_{\text {eff }}},
$$

where $D_{\text {eff }}$ is the effective electrode distance, which depends on the electrode chosen to be connected to the detection circuit. It can be computed numerically for any specific trap electrode geometry. Thus, a current of magnitude

$$
i_{\text {ind }}=q \frac{\dot{z}}{D_{\text {eff }}}
$$

flows across the connected superconducting tank circuit. Due to the large impedance of the tank circuit, when the ion is tuned into resonance, a measurable voltage drops across the resonator. The Fourier-transformed spectrum provides the sought after frequency. A typical signal is shown in Figure 7. The induced current causes dissipation in the tank circuit, leading to an effective cooling of the ion's motion with a cooling time constant

$$
\tau=\frac{m D_{\mathrm{eff}}^{2}}{q^{2} \operatorname{Re}(Z(\omega))},
$$




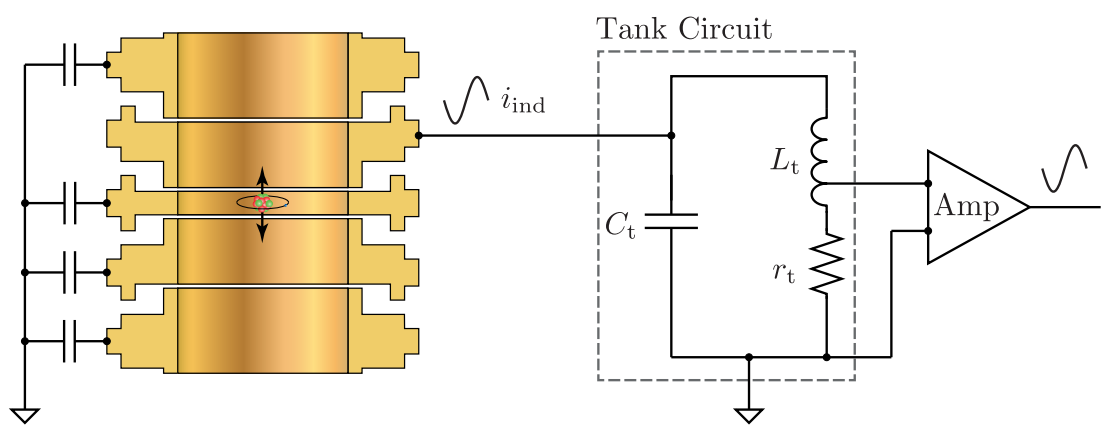

Fig. 6. Equivalent circuit of the image current detection system. The ion induces the current $i_{\text {ind }}$ into the tank circuit. Accordingly, a voltage drops across the circuit, which is detected by the cryogenic amplifier. The ion dissipates its energy into the resistance $r_{t}$, which condenses all losses of the superconducting tank circuit. Eventually, the ion comes into thermal equilibrium with the Johnson thermal noise of the tank circuit, typically at $4.2 \mathrm{~K}$.

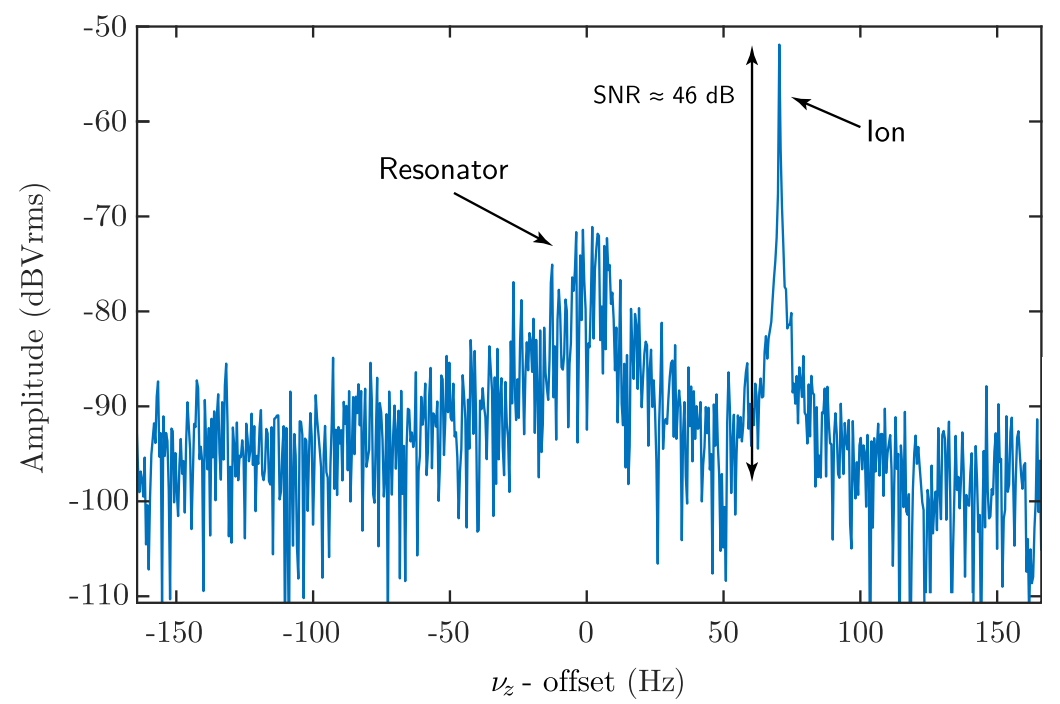

Fig. 7. A typical Fourier-transformed spectrum of a hot single ${ }^{12} \mathrm{C}^{5+}$-ion signal in a Penning trap. The axial frequency offset here is $651400 \mathrm{~Hz}$. The spectral width of the ion's peak is defined by the effective cooling time constant $\tau(\omega)$, which has been increased in this case by slightly detuning the ion from the resonator.

where $Z(\omega)$ denotes the frequency dependent impedance of the tank circuit. For HCI, typical values of $\tau$ are in the range of $10 \mathrm{~ms}$. Consequently, the ion's spectral signal is a Lorentzian to first order, with a significant finite width $\Gamma=1 / \tau$.

\subsection{Noise dip}

After cooling for several time constants, the ion eventually comes into thermal equilibrium with the tank circuit. Now, the excitation of the ion due to the thermal Johnson noise of the tank circuit exactly cancels the cooling effect. If the system is shielded against noise from external and internal sources, the ion finally adopts the temperature of the environment, typically $4.2 \mathrm{~K}$. The ion's signal does not simply 

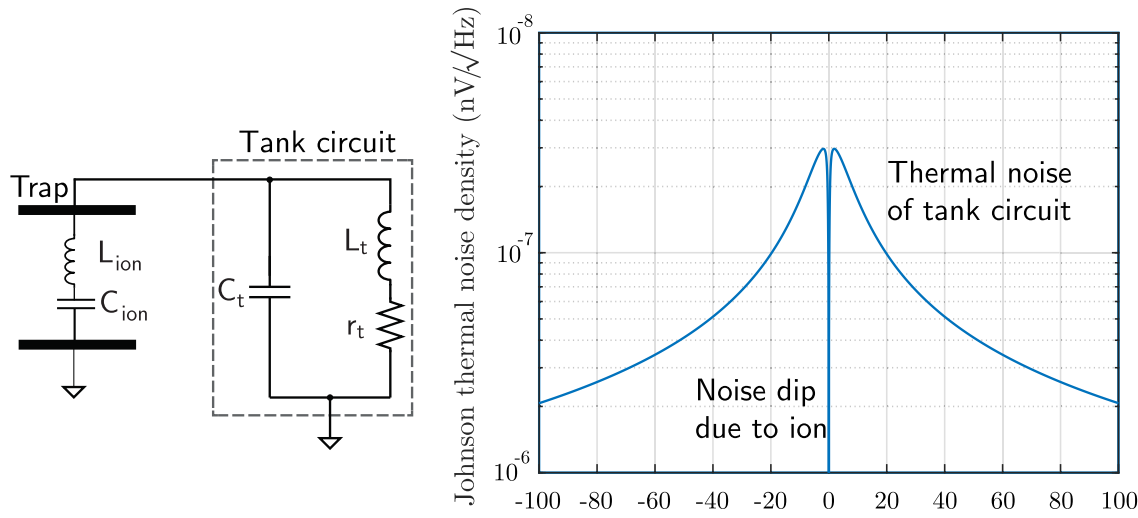

Frequency offset from resonance at $650 \mathrm{kHz}(\mathrm{Hz})$

Fig. 8. (Left) The ion in resonance with the tank circuit can be represented by a series resonant circuit with parameters $L_{\text {ion }}$ and $C_{\text {ion }}$ depending on the ion's charge and mass. (Right) The cryogenic amplifiers records the thermal noise density of the tank circuit, which features a narrow dip at the ion's axial frequency. The width of this feature is proportional to $q^{2} / m$ and amounts to typically a few $\mathrm{Hz}$. If a second or even more ions of the same species are present in the harmonic region of the trap they couple their axial frequencies and the center of mass motion consequently acts as one ion with respectively higher charge and mass. This way, the dip width can be used to measure the number of ions in the trap.

disappear within the resonators noise, but rather forms a prominent dip in the spectrum (see Figs. 8 and 29) [38]. This is because in thermal equilibrium, the ion takes a trajectory such that its induced current exactly cancels the Johnson noise at the ion's frequency. From the equations of motion we can see that the ion inside the trap is equivalent to a series resonant circuit with effective inductance $L_{\text {ion }}=\frac{m D_{\text {eff }}^{2}}{q^{2}}$ and capacitance $C_{\text {ion }}=\frac{q^{2}}{m D_{\text {eff }}^{2}} \frac{1}{\omega_{z}^{2}}$, connected in parallel to the tank circuit. Assuming that the tank circuit is the only dissipation mechanism for the ion and the ion's oscillation frequency is constant in time, the effective resonator has no series resistance, and the noise spectrum drops to zero exactly at the ion's frequency. However, since the tank circuit and the ion form a system of coupled resonators, the spectrum additionally shows a maximum if the ion's frequency is detuned with respect to the tank circuit. While the noise minimum occurs at the ion's frequency, the location of the maximum depends on the parameters of both the ion and the tank circuit and is thus subject to frequency pulling and pushing [39]. The spectral noise density can be calculated by considering the real component of the complex circuit impedance. The resulting expression can then be used to fit the measured noise spectrum and extract the undisturbed axial frequency of the ion. While the obtained precision depends on many parameters, such as the detectors signal-to-noise ratio, the measurement time and the ion type, a dip fit typically allows for a precision in the order of a few $10 \mathrm{mHz}[6]$.

\subsection{Radiofrequency coupling}

The image current detector allows us to directly measure the axial frequency. However, the modified cyclotron and the magnetron mode do not couple to the axial tank circuit due to field geometry and frequency mismatch. In certain cases it is possible to detect also the modified cyclotron motion with the help of a dedicated tank circuit, 
which then has to be tuned exactly to one specific charge-to-mass ratio. Such a detection is not possible for the magnetron motion, since any dissipation at the magnetron frequency would lead to a fast radial loss of ions due to the negative mode energy of the magnetron motion as can be seen from equation (12). It is therefore advantageous to be able to determine the radial frequencies via a coupling to the axial frequency [40]. To this end, some of the trap's electrodes are split, which allows for a number of radiofrequency excitations. The dipolar excitation, which is characterised by its position invariant form

$$
E_{D_{z}}=-\frac{U_{D_{z}}}{D_{\mathrm{eff}, z}} \cos \left(\omega_{\mathrm{rf}} t+\varphi_{\mathrm{rf}}\right)\left(\begin{array}{c}
0 \\
0 \\
1
\end{array}\right)
$$

for the axial motion, or respectively rotated for the radial modes, can resonantly drive the modes at their eigenfrequencies $\omega_{z}, \omega_{+}$and $\omega_{-}$, respectively. Here, $U_{D_{z}}$ is the peak voltage applied to the electrode, and $D_{\text {eff, } z}$ is an effective distance for the specific trap and excitation geometry. For an initially cold ion, its radius then increases linearly in time and the ion's phase lags exactly 90 degrees behind the drive's phase $\varphi_{\mathrm{rf}}$. This allows for initializing the ion's phase for the phase-sensitive measurement schemes, e.g. PnP and PnA, which will be discussed later (see Sect. 3.5). Generally, if the ion has a non-negligible initial radius, the final phase after excitation will be defined by both the initial state and the drive pulse. Consequently, in order to achieve a good control over the ion's phase, it has to be cooled to the lowest temperature possible and the excitation pulse has to be sufficiently strong to reach a radius that overcomes the thermal distribution.

The second type of frequently used excitation geometry is the quadrupolar excitation. Here, the excitation field strength depends explicitly on the position. The most frequently used geometry is the $Q_{x z}$ multipole moment with a field of the form:

$$
E_{Q_{x z}}=\frac{U_{Q_{x z}}}{D_{\mathrm{eff}, x z}^{2}} \cos \left(\omega_{\mathrm{rf}} t+\varphi_{\mathrm{rf}}\right)\left(\begin{array}{c}
z \\
0 \\
x
\end{array}\right) .
$$

Again, $U_{Q x z}$ is the peak voltage applied to the electrode, and $D_{\text {eff }, x z}$ is the effective distance now for the $Q_{x z}$ geometry. Such a field is generated by a half-split electrode, which is axially offset from the trap center. In ALPHATRAP, this is a split correction electrode. A $Q_{x z}$ excitation at any of the four sidebands $\omega_{+} \pm \omega_{z}$ and $\omega_{z} \pm \omega_{-}$can couple the two respective eigenmodes. At the red sidebands $\left(\omega_{+}-\omega_{z}\right.$ and $\left.\omega_{z}+\omega_{-}\right)$, such a coupling leads to Rabi-like oscillations of the action between the coupled modes. The coupling is adiabatic, but not energy conserving (for the ion). Rather, the peak energies of the oscillations are related by the respective frequencies:

$$
E_{ \pm, \max }= \pm E_{z, \max } \frac{\omega_{ \pm}}{\omega_{z}}
$$

Consequently, when the axial motion is in thermal equilibrium with the $4.2 \mathrm{~K}$ tank circuit, the coupling allows cooling of the radial modes to about $T_{+} \approx 160 \mathrm{~K}$ and $T_{-} \approx 52 \mathrm{mK}$. In contrast, if the ion was coupled to a cyclotron tank circuit, sideband coupling would allow cooling the axial mode to $110 \mathrm{mK}$, however this is technically much more challenging due to the typically weak coupling to the cyclotron tank circuit. In addition to the possibility to cool the radial modes, the sideband coupling also allows us to transfer phase and amplitude information between the radial and the axial mode. While the coupling is on, the axial (and radial) amplitude undergoes Rabi oscillations. Since the image current detector only sees the axial oscillation, this 
(carrier-free) amplitude modulation yields two sidebands, each offset by one Rabi frequency from the uncoupled axial frequency. The frequencies $\nu_{1}, \nu_{\mathrm{r}}$ of this doublepeak (or double-dip) show an avoided crossing with respect to the drive frequency (see Fig. 33), such that from a measurement of the double-dip spectrum and an uncoupled single-dip spectrum one can deduce the radial frequencies with similar precision as the axial frequency:

$$
\nu_{ \pm}=\nu_{\mathrm{rf}} \pm\left(\nu_{1}+\nu_{\mathrm{r}}\right) \mp \nu_{z}
$$

For most HCI, this method allows for a relative precision of typically partsper-billion per shot, which can be averaged to a few $10^{-10}$. Even better precision becomes accessible by using advanced phase-sensitive detection methods as discussed in Section 3.5, which allow for reaching 10 parts-per-trillion precision and beyond $[9,41]$.

\subsection{Phase-sensitive frequency measurements}

The noise dip described in Section 3.3 is used as a very robust tool to detect the ion's motion. One of it's main advantages lies in the fact that the ion is in thermal equilibrium with the detection circuit during the measurement. This way, systematic shifts due to the kinetic energy of the ion are typically negligible. However, since the ion is driven by the thermal noise, its motion is necessarily incoherent. Correspondingly, the statistical precision of the fitted frequencies scales unfavorably slow with the averaging time $T: \frac{\delta \nu_{z}}{\nu_{z}} \propto \frac{1}{\sqrt{T}}$. In addition, though the lineshape of the noise dip is well established, it depends on the transfer function and the technical parameters of the detection system, which are not known to arbitrary precision. Depending on the ion and the detector parameters, this can set stringent limits to the achievable accuracy. These disadvantages can be overcome by using a coherent, phase sensitive detection. Here, the ion is initially prepared in a coherent state with well-defined phase $\phi_{0}$. This can be readily achieved by using a resonant, dipolar rf-excitation (see Sect. 3.4) on one of its eigenfrequencies. Afterwards, the ion is left undisturbed and can be completely decoupled from the detector for a variable phase evolution time $T_{\text {evol }}$. Finally, the ion's final phase $\phi_{1}$ is measured using the complex Fourier transform of a short transient of the ion, recorded after bringing the detector into contact with the ion again. Now, the frequency $\nu$ of the ion's free evolution can be determined by assuming

$$
\phi_{1}=2 \pi \nu T_{\mathrm{evol}}+\phi_{0}
$$

Consequently, $\nu$ can be deduced with a precision of

$$
\frac{\delta \nu}{\nu}=\frac{\sqrt{\delta \phi_{0}^{2}+\delta \phi_{1}^{2}}}{2 \pi \nu} \frac{1}{T_{\mathrm{evol}}} .
$$

For the axial frequency this scheme can be directly applied and has been routinely utilised in the predecessor experiment of ALPhATRAP in Mainz in order to determine the small shift in the axial frequency due to a spin-state change via the continuous Stern-Gerlach effect, which will be introduced in Section 3.6. Here, systematic shifts arising from the relatively large amplitudes required to detect the phase with sufficient resolution are of minor concern. For the precise determination of the cyclotron frequency however, such shifts can be unacceptably large. Furthermore, a direct detection of the cyclotron phase requires a tank circuit in resonance with the cyclotron 

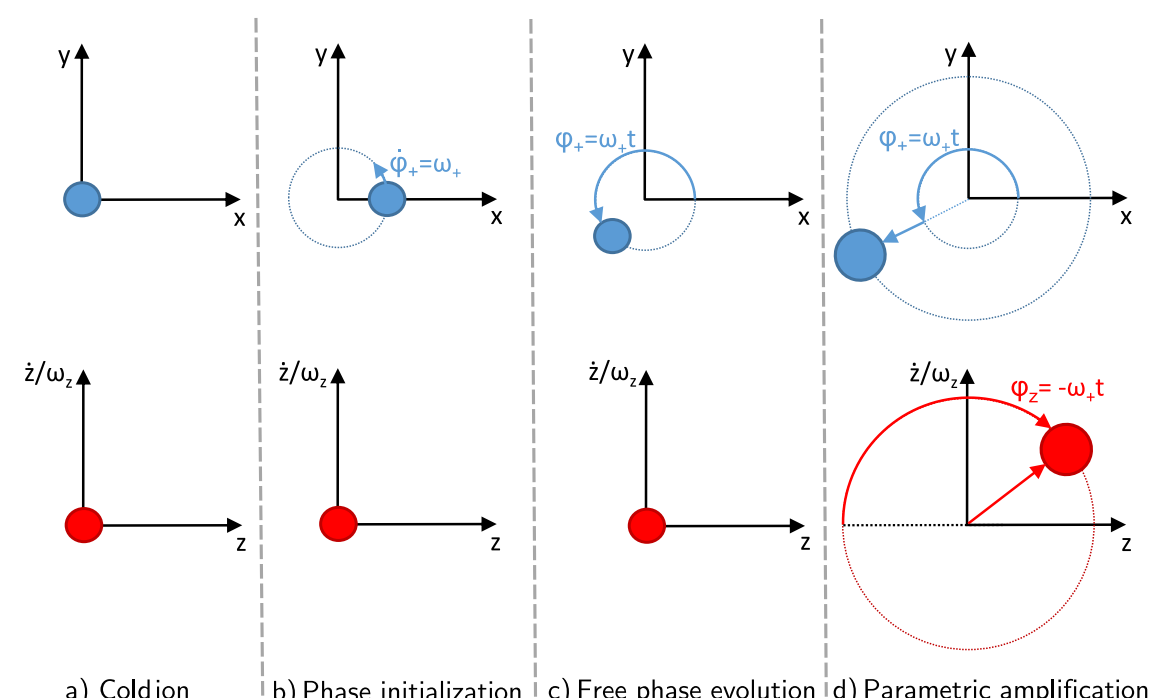

Fig. 9. Sketch of a PnA cycle. The ion is initially cooled in axial and cyclotron modes (a). Then the ion is excited by a dipolar excitation pulse, which initialises the ion's cyclotron phase $\varphi_{+}$. The required amplitude is determined only by the cold ion's energy distribution and can be generally smaller than the threshold energy of the detector. (b) In the phase evolution period, the ion is entirely decoupled from all detection circuitry. The phase $\varphi_{+}(t)=\omega_{+} t$ accumulates (c). Finally, the cyclotron phase is imprinted onto the axial motion via a radiofrequency coupling at the "blue" cyclotron-axial sideband. There, it can be read-out with good signal-to-noise ratio using the image-current detector (d).

frequency of the specific ion. Such a tank circuit however would lead to systematic frequency shifts on its own via frequency pulling effects. Correspondingly, a technique ([42], see Fig. 9) has been developed that allows us to transfer the cyclotron phase information onto the axial motion, such that it can be read out on the axial tank circuit. For PnA, at the end of the evolution time the cyclotron motion is coupled to the axial one via a quadrupole excitation on the "blue" sideband $\nu_{\text {drive }}=\nu_{+}+\nu_{z}$. This way, not only the phase information is transferred, but simultaneously the axial and cyclotron motions are resonantly amplified to virtually arbitrary amplitudes, that can be read out with good signal-to-noise ratio, even if the initial motional amplitude is below the detection threshold of the image current detector. Consequently, systematic shifts due to the excitation amplitude can be extremely low if the ion is initially cold. Since the measured phase directly corresponds to the cyclotron frequency, the impact of uncertainties of the axial frequency, e.g. due to voltage fluctuations or imperfections of the lineshape model, is drastically reduced by a factor $\frac{\nu_{z}}{\nu_{+}} \approx 1 / 50$. This is a key factor for achieving uncertainties exceeding about 0.1 parts per billion (ppb) for the $g$-factor measurement.

\subsection{The continuous Stern-Gerlach effect}

The introduced methods allow for the precise measurement of the trap eigenfrequencies, and thus, via the invariance relation (13), for the determination of the free-space cyclotron frequency $\nu_{c}$. For a $g$-factor measurement however, also the Larmor precession frequency of the spin has to be determined. Since the spin precession is not accompanied by a charge oscillation, it cannot be seen by the image current detector. 
Rather, it is determined by performing spin-flip spectroscopy on the Zeeman transition. A millimeter-wave excitation at the Larmor frequency can resonantly induce quantum jumps between the two spin eigenstates. Such transitions of a single spin can then be detected using the continuous Stern-Gerlach method. Here, a quadratic magnetic inhomogeneity of the form

$$
B_{z}(z, \rho)=B_{0}+B_{2}\left(z^{2}-\frac{\rho^{2}}{2}\right)
$$

is superimposed to the trap's homogeneous magnetic field, which causes a small additional harmonic axial force due to the magnetic moment of the spin

$$
F_{z}= \pm \mu_{z} \partial_{z} B_{z}
$$

Combined with the electrostatic trapping field, the additional force of this magnetic bottle causes a slight, but detectable frequency change depending on the magnetic moment projection on the trap axis $\mu_{z}$ :

$$
\Delta \nu_{z}=\frac{B_{2}}{(2 \pi)^{2} m \nu_{z}} \mu_{z}
$$

From equation (25) one can see that the frequency shift scales linearly with the strength of the magnetic bottle $B_{2}$ and inversely with the ion's mass $m$. In order to be able to determine the spin state even of heavy HCI it is thus mandatory to achieve a sufficiently strong bottle. In the ALPHATRAP experiment we have manufactured the central ring electrode of the analysis trap (AT, see Sect. 3.7), which is dedicated to the spin state detection, from a ferromagnetic cobalt-iron alloy (VACOFLUX50 [43]), which yields a magnetic bottle of $B_{2} \approx 44 \mathrm{kT} / \mathrm{m}^{2}$. This is significantly stronger than in previous experiments on highly charged ions, but a spin-flip still only causes an axial frequency jump of $2 \Delta \nu_{z} \cong 154 \mathrm{mHz}$ for the heavy, hydrogenlike lead ${ }^{208} \mathrm{~Pb}^{81+}$. While this would be easily resolvable in a normal precision Penning trap, the strong magnetic bottle not only causes axial frequency changes due to the spin state, but the radial motions of the ion, magnetron and specifically modified cyclotron motion, also induce an axial magnetic moment projection. Thus, the axial frequency in the magnetic bottle is strongly dependent on the cyclotron mode energy, leading to significant drifts if the cyclotron energy is not kept perfectly constant in time:

$$
\Delta \nu_{z}=\frac{B_{2}}{(2 \pi)^{2} m \nu_{z}}\left(g m_{z} \mu_{\mathrm{B}}+\frac{E_{+}}{B_{0}}-\frac{E_{-}}{B_{0}}\right),
$$

where $m_{z}$ denotes the quantum number of the spin projection onto the axis defined by the magnetic field. Any kind of spurious noise on the electrodes will cause a random walk of the cyclotron energy, and must thus be categorically avoided by using suitable filtering techniques on all voltage and signal lines.

A special challenge is imposed by measurements of boronlike ions. Since the ground-state of these ions is a $p_{1 / 2}$-state, their Landé $g$-factor is about a factor of 3 smaller than for a comparable hydrogenlike or lithiumlike ion. This translates in the extreme case of boronlike lead ${ }^{208} \mathrm{~Pb}^{77+}$ into a frequency jump of only $53 \mathrm{mHz}$, which can only be reliably detected by using special phase-sensitive measurement techniques.

An important advantage of the spin-state detection via the continuous SternGerlach effect is that virtually any ion with arbitrary charge $q$ and mass $m$ can be measured. Unlike in the case of laser-based double-resonance techniques [44], no 
optically accessible transition with suitable lifetime is required. Furthermore, any single transition can be detected, virtually independent of the lifetime of the excited state, if only the transition changes the axial spin projection. This is an important prerequisite for performing ultra-high precision laser spectroscopy of highly charged ions at Alphatrap, as will be discussed in Section 6.3.

\subsection{The double-trap technique}

The large magnetic bottle in the analysis trap, which is required to make the spinstate visible, causes strong, undesired shifts of all trap eigenfrequencies. This imposes a significant limitation for the achievable precision. For example, the thermal axial motion at $4.2 \mathrm{~K}$ would broaden the Larmor resonance inside the magnetic bottle to a relative width of several parts-per-million [45], depending on the mass of the ion. To solve this problem $[6,46]$, the measurement is distributed into two specialised traps: While the analysis trap features the strong magnetic bottle, which enables spin-state detection, all precision spectroscopy is performed on the same ion inside the so-called precision trap (PT). This trap is spatially separated from the analysis trap and has a drastically lower inhomogeneity. At ALPHATRAP, we have additionally placed ferromagnetic material such that it exactly compensates the inhomogeneity in the precision trap originating from the magnetic bottle in the analysis trap. The residual inhomogeneity in the precision trap then is almost 6 orders of magnitude lower than in the analysis trap (see Sect. 4.5), allowing for extremely high-precision spectroscopy. The measurement process then starts in the analysis trap, where the ion's spin state is determined. Subsequently, the ion is transported into the precision trap by adiabatically shifting the electrostatic trap center with a stack of transport electrodes and adequate voltages. In the precision trap, the eigenfrequencies are measured and simultaneously a millimeter-wave excitation is irradiated into the trap. If this excitation is sufficiently close to the ion's Larmor frequency, the spin state can be changed resonantly. After transporting the ion back to the analysis trap, the spin state is read out and compared to the initial state. One measurement cycle thus gives the cyclotron frequency of the ion and the randomly chosen frequency of the simultaneously irradiated millimeter-wave excitation, together with the information whether or not a spin-flip has occurred. The ratio of the two frequencies in measurement step $i$ is typically termed $\Gamma_{i}$. In combination with the spin-flip information, any measurement cycle is self-contained and does not depend explicitly on the magnetic field or on fluctuations of the field between successive cycles. Finally, the spin-flip probability (or fraction) can be plotted as function of $\Gamma \equiv \frac{\nu_{\mathrm{MW}}}{\nu_{\mathrm{c}}}$, which reveals (after small corrections for systematic shifts) a resonance maximum at $\Gamma_{0} \equiv \frac{\nu_{\mathrm{L}}}{\nu_{\mathrm{c}}}$, from which the $g$-factor can be calculated.

In order to being able to apply the double-trap technique, it is mandatory to have an unambiguous spin-state detection. One important milestone is therefore to push spurious frequency fluctuations $\delta \nu_{z}$ in the analysis trap below a threshold of about $\delta \nu_{z} \ll \Delta \nu_{z}$, or about $100 \mathrm{mHz}$ in the case of ${ }^{208} \mathrm{~Pb}^{81+}$. Apart from efficient filtering of noise, also the radial (specifically cyclotron) temperatures have to be kept as low as possible, as the effective axial frequency fluctuations depend linearly on the cyclotron energy $[47,48]$.

\subsection{Laser cooling}

One of the limitations of previous measurements performed in Penning traps have been systematic frequency shifts and uncertainties which depend on the ion's energy 
and amplitude [42], see Section 4.5. We would like to reduce these shifts by utilizing laser cooling techniques. Further cooling would mean a reduction of the ion's energy and motional amplitudes, up to the point the ions are arranging themselves in a Coulomb crystal. This leads to two positive effects: the smaller the coordinate space the fewer anharmonicities of the trapping field are seen by the ions, which is especially interesting for a configuration with more than one ion in the trap, and also the reduction of momentum space leads to a reduction of velocity-depended shifts due to second-order Doppler effect and relativistic mass increase. The formation of a Coulomb crystal opens up new measurement schemes (see Sect. 6) and enables spatial control for two or more ions. In general, laser cooling is only applicable to such ions in which a suitable transition is addressable for a laser. Since the HCI which are envisaged to be used in ALPHATRAP generally do not have strong optical transitions, sympathetic cooling of the HCI by another laser cooled ion has to be used. The final temperature of the HCI should become close to that of the directly laser cooled light ion, in case there are no further heating mechanisms present. Sympathetic laser cooling in a Penning trap between two different ion species was first shown in 1986 for ${ }^{198} \mathrm{Hg}^{+}$ions sympathetically cooled by ${ }^{9} \mathrm{Be}^{+}$ions [49]. Laser cooling relies on the momentum transfer by light onto massive particles upon photon absorption and emission. The interaction with the laser light of frequency $\omega_{\text {Laser }}$ respectively wavelength $\lambda$ can be described by scattering events. If the detuning of the laser compensate for the Doppler shift experienced by the ion moving towards the laser beam, the ion can absorb a resonant photon upon which its momentum gets reduced by $\hbar k$, where $k$ is the angular wave number $k=\frac{2 \pi}{\lambda}$ of the incident radiation. Since the spontaneous reemission is isotropic, the recoil momentum over a large number of emission cycles averages to zero, so the net effect over many cycles results in a reduction of the momentum and thus for the axial and cyclotron mode also of the motional amplitudes.

Even though the average recoil momentum over a large number of emission cycles is zero, the mean squared momentum is not. The cooling limit can be derived by using rate equations for parametrizing the interaction into damping and fluctuating forces. The steady state is reached when these cooling and heating forces are equal and one obtains the well-known Doppler cooling limit at a temperature $T_{D}$

$$
T_{D}=\frac{\hbar \gamma}{2 k_{\mathrm{B}}}
$$

For ${ }^{9} \mathrm{Be}^{+}$the usable cooling transition between the ${ }^{2} \mathrm{P}_{3 / 2}, m_{J}=3 / 2$ and ${ }^{2} \mathrm{~S}_{1 / 2}, m_{J}=$ $1 / 2$ states has a lifetime of $\tau=8.1(4) \mathrm{ns}$ and a line width of $\gamma=1 / \tau=19.6 \mathrm{MHz}$ [50] and thus the Doppler cooling limit $T_{D}$ is about $0.5 \mathrm{mK}$. This temperature is two orders of magnitude lower than achieved so far by using feedback cooling [51]. Even if the concept of a temperature in the sense of an ensemble average defined often in thermodynamics might not be suitable for a single ion in a Penning trap, the temperature of an ion's motional mode can be defined via the time averaged expectation value of the energy within the meaning of the ergodic hypothesis as

$$
T_{z,+,-}=\frac{\left\langle E_{z,+,-}\right\rangle}{k_{\mathrm{B}}},
$$

which also over several measurements will sample a thermal Boltzmann distribution.

For laser cooling in a Penning trap some special peculiarities occur and have to be accounted for, e.g. see [52]. Since the motional frequencies of the three eigenmotions are independent either they would need to be cooled individually or a coupling between the modes for an energy transfer has to be established while at least one mode 
is laser cooled (compare Sect. 3.4). In the ALPhATRAP experiment only axial access for the laser is possible and such a scheme for mode coupling has to be employed. Care has to be taken for the magnetron mode, in which the radius increases for lower total magnetron energy and thus becomes unstable. As a result of the harmonic motion of the ion with relatively large oscillation frequencies, laser cooling in a Penning trap can also be extended to resolved sideband cooling $[53,54]$ in the so called Lamb-Dicke regime. In the case the cooled ion has a motional amplitude below the wavelength of the cooling laser and the used cooling transition has a line width much smaller than the motional frequency of the cooled mode $\left(\Gamma \ll \nu_{z}\right)$, the spectrum of the ion shows a strong central carrier frequency at $\nu_{o}$ with equally spaced sidebands and frequency offset by the motional frequency of a mode, e.g. $\nu_{z}$. In this case, by driving the "red" sideband at $\nu_{\text {red }}=\nu_{o}-\nu_{z}$, individual motional quanta can be removed and eventually the ion can be cooled into the motional ground state [55].

At Alphatrap ${ }^{9} \mathrm{Be}^{+}$was chosen as laser cooled ion since it provides good optical access and a comparably simple cooling cycle. Due to the strong magnetic field the Zeeman splitting is given by the Paschen-Back effect: The fine structure and hyperfine levels are strongly split in the order of several tens of $\mathrm{GHz}$ [56,57]. This splitting of the optical transitions is larger than either the Doppler or natural broadening so the laser has to be tuned to a specific transition between two hyperfine manifolds. In the case of ${ }^{9} \mathrm{Be}^{+}$a suitable transition is between the ${ }^{2} \mathrm{P}_{3 / 2}, m_{J}=3 / 2$ and ${ }^{2} \mathrm{~S}_{1 / 2}, m_{J}=1 / 2$ levels, which is in the presence of the strong axial magnetic field a circular polarised transition and in the UV range at a wavelength of about $313 \mathrm{~nm}$.

\section{Experimental setup}

\subsection{Overview of the Alphatrap apparatus}

The main goal of ALPHATRAP is performing high-precision measurements of the $g$ factor of heavy, highly charged ions. While large parts of the apparatus are based on the $g$-factor experiment on highly charged ions at the Johannes Gutenberg-University in Mainz, Germany, the production of heavy HCI with ionization energies of up to $100 \mathrm{keV}$ necessitates a dedicated production facility outside of the Penning-trap chamber. Today, only few such facilities exist world-wide. Among these are the experimental storage ring (ESR) at the GSI in Darmstadt, Germany, where large numbers of heavy HCI are routinely produced by stripping stored ions in a target foil at kinetic energies of order $400 \mathrm{MeV} / \mathrm{u}$. However, the resulting HCI do still have a large kinetic energy and energy spread and have to be cooled in the dedicated HITRAP [58] facility, which is currently under development. On the other end, there are large electron-beam ion traps (EBITs), where electron beam energies in excess of the required $102 \mathrm{keV}$ for ${ }^{208} \mathrm{~Pb}^{81+}$ (or even $132 \mathrm{keV}$ for ${ }^{238} \mathrm{U}^{91+}$ ) are feasible. Here, the HCI are produced nominally at rest, albeit still at a temperature in the order of a few $\mathrm{eV} \times \mathrm{q}$. While this energy spread is still too large for high-precision experiments, such ions can be extracted from the EBIT and recaptured in a Penning trap, where they are subsequently resistively cooled to $4 \mathrm{~K}$. One such EBIT is the cryogenic Heidelberg EBIT at the MPIK. To deliver HCI from the Heidelberg EBIT to the AlPhatraP Penning traps, a room-temperature electrostatic beamline was built that allows injecting ion bunches. Alternatively, the beamline can also be used to inject HCI from a smaller, room-temperature Heidelberg Compact Electron Beam Ion Trap (HC-EBIT) [59], which allows "offline" production of medium heavy HCI, as well as from a laser ion source (LIS) which can deliver singly-charged ${ }^{9} \mathrm{Be}^{+}$ions for sympathetic laser cooling of HCI. A schematic top-view of the facility is shown in Figure 10. 


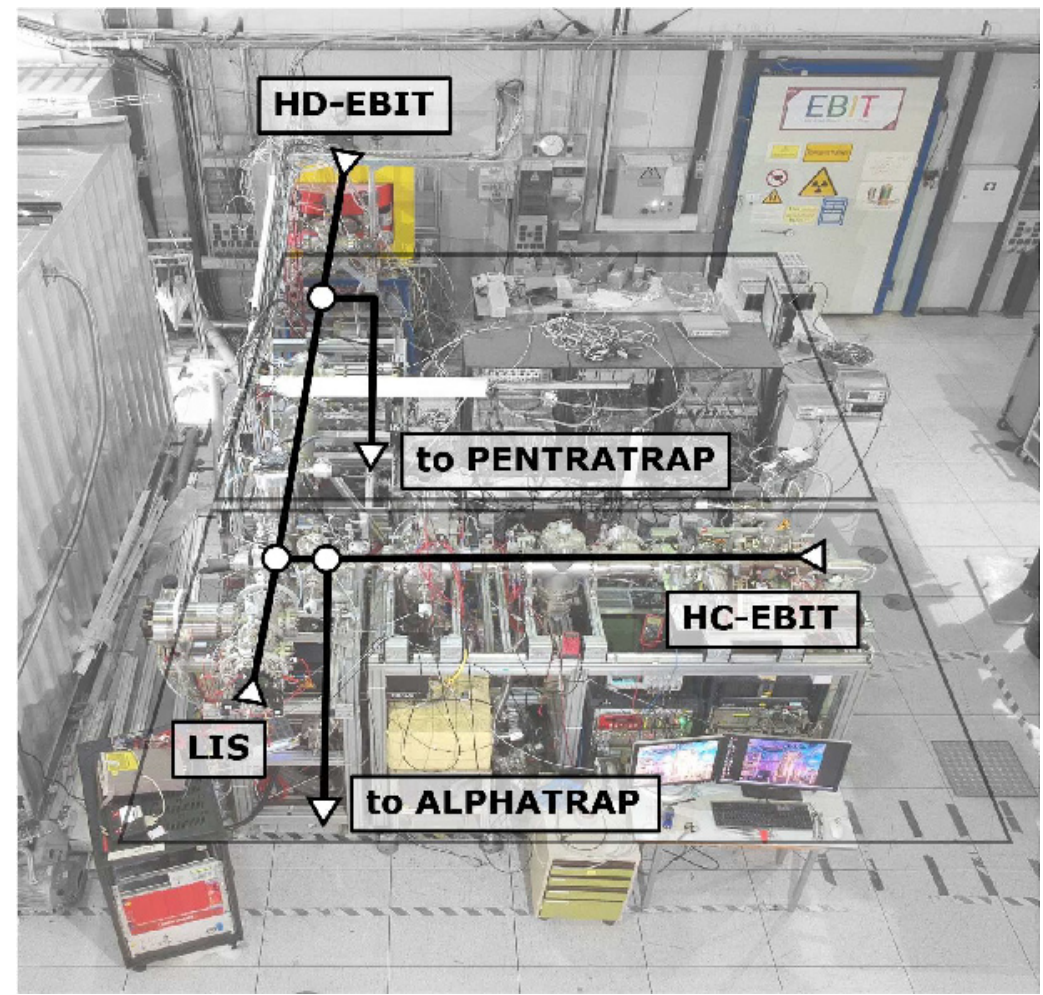

Fig. 10. Overview of the experimental setup. Heavy HCI are produced in the Heidelberg EBIT (HD-EBIT) and transported electrostatically to either the PENTATRAP mass spectrometer [60,61], which has recently started taking data, or the ALPHATRAP $g$-factor experiment. AlPHATRAP additionally is equipped with a room-temperature HC-EBIT, which can deliver medium charged ions and a laser ion source (LIS), which is dedicated to the production of ${ }^{9} \mathrm{Be}^{+}$ions for laser cooling.

\subsection{Cryostat and magnet}

The Penning traps of the Alphatrap setup are located inside a superconducting magnet which provides the homogeneous and temporally stable magnetic field. Here, we reuse an Oxford 200/130 NMR vertical bore magnet, which has previously been used in the SMILETRAP experiment [62]. It is capable of providing up to $4.7 \mathrm{~T}$, but has been charged to only $4 \mathrm{~T}$. This choice brings the Larmor and cyclotron frequencies into a convenient regime. The superconducting magnet has a self-shielding factor of only 14.3 and is therefore supplemented by a superconducting self-shielding coil around the trap chamber, which helps to strongly suppress the effect of external magnetic field fluctuations in the trap. The homogeneity of the bare-magnet field is shimmed to about $2 \times 10^{-7}$ based on the lineshape of a $1.5 \mathrm{~cm}^{3}$ cylindrical NMR probe. The superconducting magnet is of warm-bore type, such that the experiment needs an additional, independent liquid helium cryostat. Despite the additional complexity, this has a number of practical advantages: Firstly, the trap vacuum chamber is surrounded by an insulation vacuum, which significantly softens the requirements on the leak-tightness. Secondly, the superconducting electronics can be inserted into the magnetic field above the critical temperature and cooled within the field. The magnet is supported on a table which rests on rails. This allows us to move the magnet and apparatus to the side of the beam-line and remove the $4 \mathrm{~K}$ insert for 
maintenance and modifications without the need to vent the UHV beam-line, which enables cycling the experiment on time scales of about one week, or even shorter if required.

\subsubsection{Cryostat}

Since the use of closed-cycle coolers is unfavorable due to unavoidable vibrations, which affect the frequency stability, we have designed a liquid helium cryostat specifically for Alphatrap. Its design is sketched in Figure 11. On top of the magnet, supported by spring-loaded posts, sits the liquid nitrogen $\left(\mathrm{LN}_{2}\right)$ tank which contains $55 \mathrm{l}$ of $\mathrm{LN}_{2}$, sufficient to keep the experiment cold for about 5 days. A copper shield tube extends into the superconducting magnet's bore and provides the thermal shielding of the inner $4 \mathrm{~K}$ insert, which hangs vertically on three Vespel SP1 rods for minimum heat conductance. All other connections to the $4 \mathrm{~K}$ section, including the beamtube and the filling ports, are flexible to prevent varying stress due to thermal shrinking.

While the $4 \mathrm{~K}$ part can be easily removed by a crane, the $77 \mathrm{~K}$ cryostat is permanently mounted. To provide optimal radiation shielding, the $\mathrm{LN}_{2}$ cryostat is completed by a piece of copper shield, which is mounted on the $4 \mathrm{~K}$ insert. It is attached by copper braids and copper-beryllium springs to the $\mathrm{LN}_{2}$ cryostat just before the final lowering of the $4 \mathrm{~K}$ insert. The complete $77 \mathrm{~K}$ stage is insulated by vacuum and multi-layer insulation (MLI). The $4 \mathrm{~K}$ stage, which is mainly subjected to conductive load via the electric cabling, is radiation-shielded by a high-conductivity, single layer metal foil, which minimises absorption of heat radiation from the $77 \mathrm{~K}$ stage. The overall load on the $4 \mathrm{~K}$ stage, including dissipated power in the cryogenic amplifiers, is below $100 \mathrm{~mW}$, resulting in a hold-time of about 5 days with $14 \mathrm{l}$ of LHe.

To achieve maximum frequency stability, the cryostat has been optimised for minimal susceptibility to external influences. The top flange of the cryostat, called the "hat", which holds the electric feedthroughs for the cryogenic electronics and which supports the $4 \mathrm{~K}$ insert, is constructed such that metrological pressure variations have minimal impact on the relative position of trap and magnet coils. If required, further stabilization can be achieved in the future via piezoelectric elements, which are installed in the support structure and which allow moving the $4 \mathrm{~K}$ insert vertically by up to $20 \mu \mathrm{m}$.

\subsubsection{Beamtube and cryovalve}

The externally produced ions are injected into the cryogenic trap chamber via a beamtube. After the last steering lens on top of the cryostat there are no further ion optical elements inside this tube other than the magnetic field of the superconducting magnet which guides the ions into the trap. Since the trap vacuum is connected to the room-temperature beamline, there are severe requirements on the vacuum quality. In order to being able to store heavy HCI for extended time periods in excess of hours, a vacuum better than $10^{-16}$ mbar should be achieved. This is only possible due to the cryogenic freeze-out of almost all gases, with the important exceptions of helium and hydrogen. These two gases have a sizeable vapor pressure even at $4.2 \mathrm{~K}$, but they can be adsorbed on other, particularly metallic, surfaces in single or few atomic layers. Consequently, if the amount of those gases inside the trap chamber can be limited below a complete monolayer coverage, a virtually complete rest-gas freeze-out occurs. However, the room-temperature beamline continuously feeds particularly hydrogen molecules, which diffuse from the bulk of the stainless steel walls, even though all 


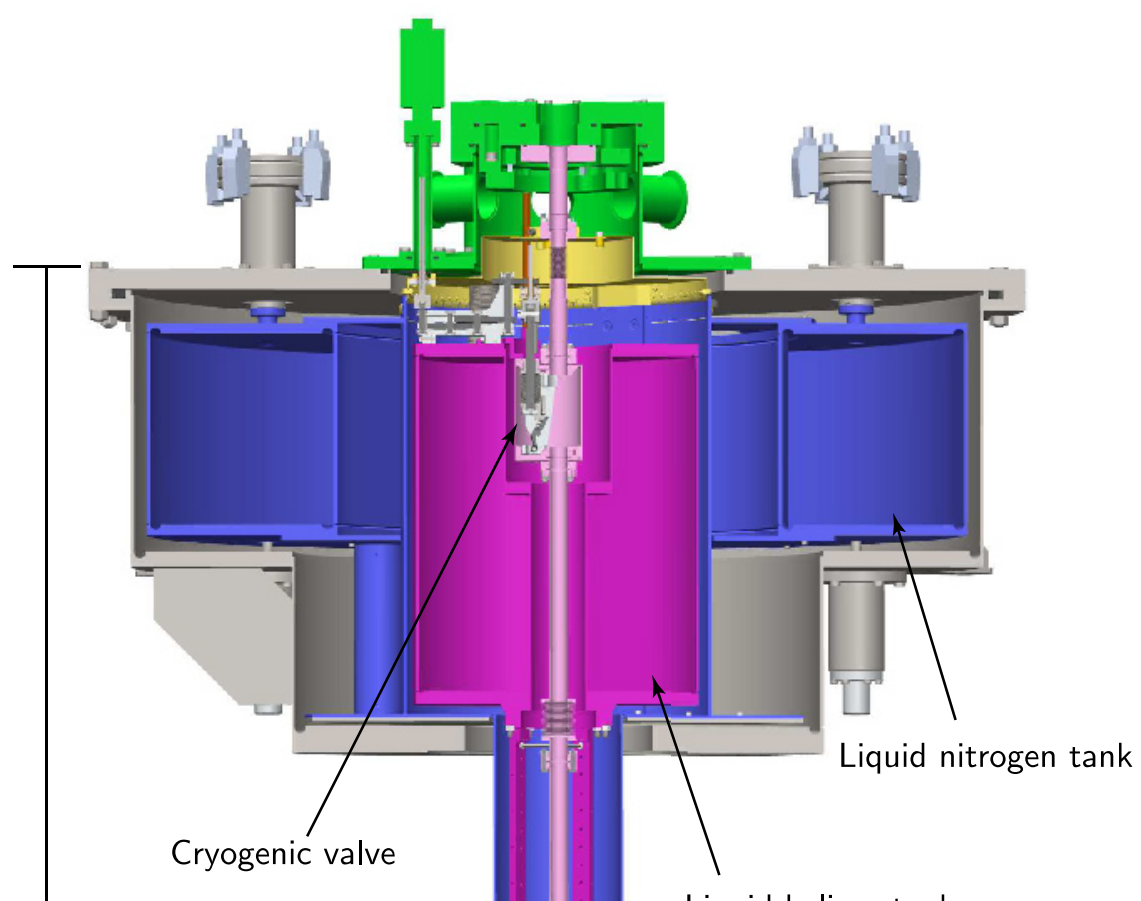

Liquid helium tank

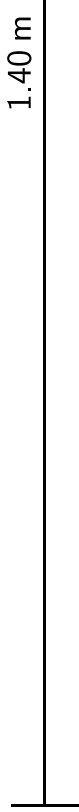

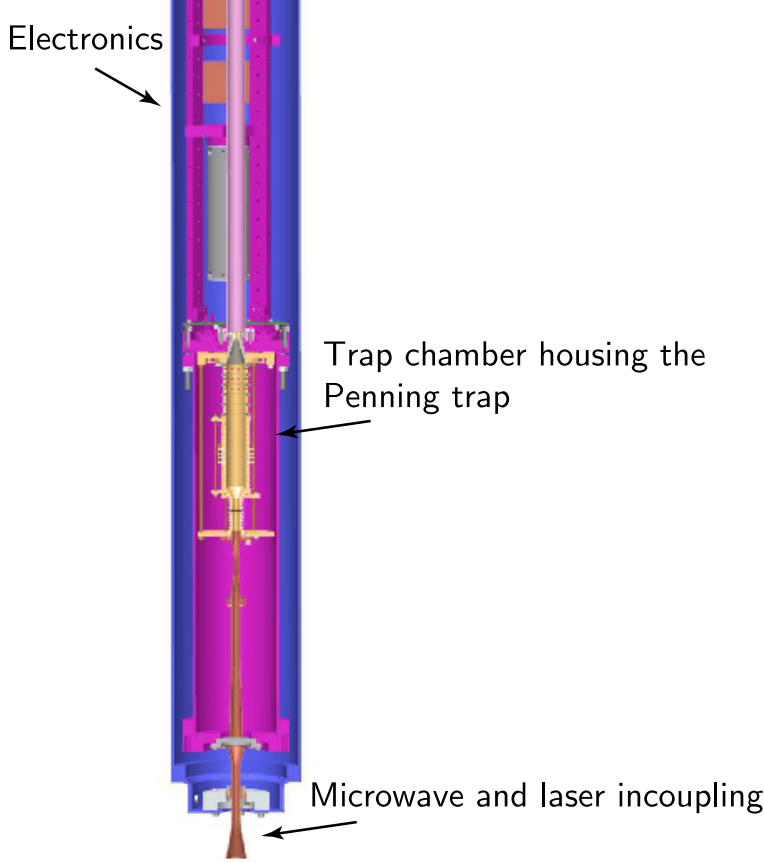

Fig. 11. The Alphatrap cryostat. The $4 \mathrm{~K}$ (pink) insert with the trap chamber and the cryogenic electronics is supported via low-conductance rods from the so-called hat (green). Here, all electric connections enter the insulation vacuum via multi-pin feedthroughs. The $77 \mathrm{~K}$ shield (blue) is completed by a top piece which is lifted out of the magnet together with the $4 \mathrm{~K}$ insert. 
beamline parts have been vacuum-fired to minimise the amount of dissolved hydrogen. By considering the vacuum conductance of the cryogenic part of the beamline even the achieved rest-gas pressure in the room-temperature beamline of typically $10^{-11} \mathrm{mbar}$, leads to a limited operation time of the experiment. After the trap chamber has accumulated enough gas to form a monolayer, the vacuum would degrade drastically. In order to maximise this time and to become less vulnerable to temporary vacuum problems in the room-temperature section, a cryogenically operated valve has been designed and installed at Alphatrap. When closed, this valve, which is cooled to $4.2 \mathrm{~K}$, provides a virtually complete blockage of gas flux into the trap chamber, and thus eliminates also the direct flux of gas from the room-temperature section to the ions. Only in the short intervals while ions are injected gas can enter the trap chamber. This way, a virtually unlimited operation can be achieved, a key requirement for the envisaged experiments with heavy HCI. The valve is operated manually via a rotational feedthrough at the hat, which is coupled on demand (to minimise heat conductivity while not operating the valve) via a hex-key to a gearbox mounted on the LHe tank. The gearbox operates a flap inside the valve housing via a linear feedthrough realised with a membrane bellow. The successful routine operation of this valve has been demonstrated in the 2017 commissioning run and a vacuum in excess of $10^{-17}$ mbar has been demonstrated by storing a single ${ }^{40} \mathrm{Ar}^{13+}$ ion over more than 2 months without any charge exchange. Alphatrap is the first experiment world-wide to demonstrate virtually infinite storage time of arbitrary, externally injected HCI.

\subsubsection{Superconducting self-shielding coil}

Measurements envisioned in AlPhATRAP take place on different timescales over which a stable magnetic field is desirable. On short timescales of seconds to minutes individual frequency measurements are performed whereas a whole measurement campaign can take up to several weeks, therefore temporal magnetic field stability is essential for the experiment. Activities in the facilities around the measurement setup are sources for external magnetic field perturbations and fluctuations and cause temporal deviations of the ion's frequencies. In order to mitigate homogenous axial magnetic field fluctuations a self-shielding superconducting solenoid [63] is wound around the cylindrical trap chamber body. The aspect ratio and positioning of the solenoid is optimised to maximise shielding from external field fluctuations at the position of the ion inside the precision trap. The solenoid itself is wound from single stranded and formvar insulated niobium-titanium (NbTi) wire. The two ends of the coils are welded together and wrapped around a heating resistor, which can be used to quench a persistent shielding coil current. The shielding of the magnet can be estimated from the shift to be expected knowing the strength of the applied external magnetic field and is $\approx 14.3$. Measurements show that external field fluctuations are reduced by the additional self-shielding coil by an additional factor of $\approx 6.7$. Figure 12 shows the frequency shift in the modified cyclotron frequency $\nu_{+}$in dependence of the externally applied magnetic field. This value is well below values one estimate according to [63]. The reason for this is currently under investigation and could be due to slightly intolerances in the alignment or the mutual inductances between the shielding coil and coils of the magnet, namely the main field coil and especially gradient shimming coils.

\subsection{The Alphatrap beamline}

The production of heavy HCI up to hydrogenlike lead ${ }^{208} \mathrm{~Pb}^{81+}$ in the cryogenic Penning-trap structure itself is technically very challenging due to the high voltages 

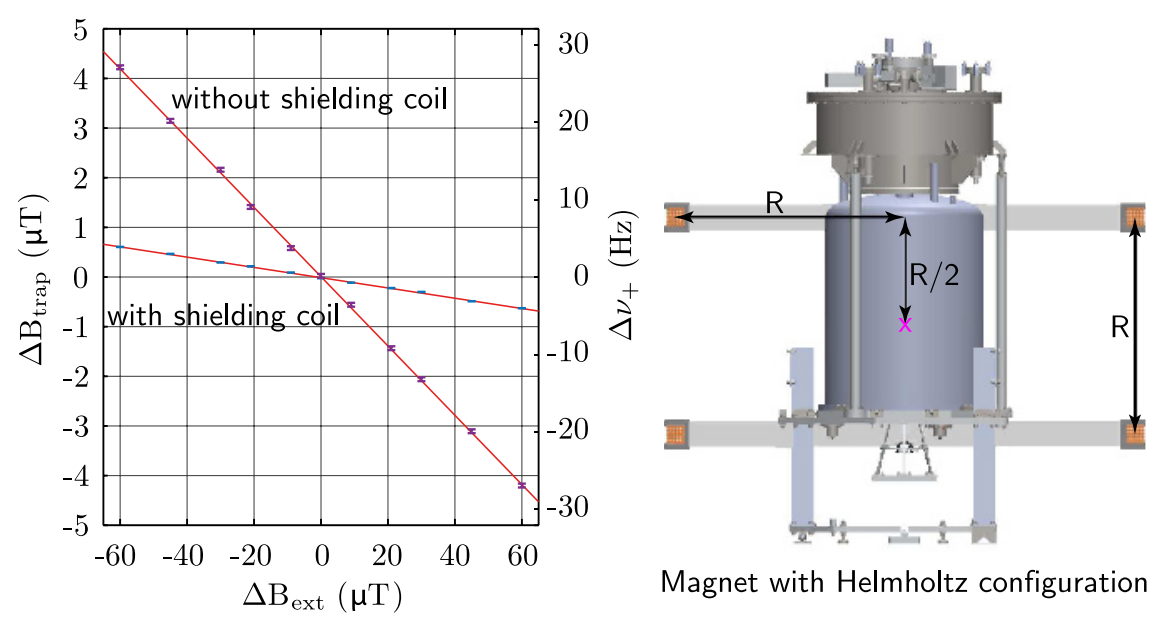

Fig. 12. Determination of the shielding factor of the superconducting solenoid. Via a double-dip (see Sect. 5.3) measurement the modified cyclotron frequency was measured. The magnetic field was produced by a pair of solenoid coils around the magnet. The coils were used in a Helmholtz configuration to apply a homogenous magnetic field perturbation wirth known strength at the center of the trap. The homogeneity $\delta B_{z} / \overline{B_{z}}$ along the trap and magnet center axis is estimated to be better than $1 \times 10^{-4}$ over the length of the superconducting self-shielding coil and $1 \times 10^{-2}$ over the estimated length of the main coils of the superconducting magnet. On the right hand side, a sketch of the Helmholtz configuration setup around the magnet is shown. The pink cross indicates the position of the ion in the center of the precision trap. The two coils are arranged concentric around the magnet with an axial spacing identical to their radius $R=90 \mathrm{~cm}$ symmetrically with respect to the nominal precision-trap center. Each coil has around 600 windings and the pair generates a magnetic field of $0.6 \frac{\mu \mathrm{T}}{\mathrm{A}}[64]$.

necessary to reach the high electron energies. Therefore, the production of these ions is performed in a dedicated external ion source. The Heidelberg EBIT [59] is designed to reach electron energies of up to $150 \mathrm{keV}$. To transfer the HCI from the Heidelberg EBIT and inject them into the AlphatraP setup, an ultra-high vacuum (UHV) beamline has been designed and set up. The beamline opens the possibility to connect further ion sources such as the HC-EBIT, which is used for the production of low and medium mass HCI as well as the commissioning of the ion injection system. Furthermore, a laser ablation ion source has been developed to provide singly charged beryllium ions for sympathetic laser cooling of HCI into ALPHATRAP. All external ion sources will be described in more detail in the next sections.

The foreseen experiments with single HCI require extremely low background gas pressure to reduce the probability of ion loss due to charge-exchange reactions with neutral rest gas particles. Such vacuum conditions have been successfully achieved in several "hermetically closed" cryogenic systems $[6,9,41,65-67]$, i.e. systems which only consist of an evacuated, cryogenic experimental zone or systems in which this zone is separated from the main room temperature adjacent parts by a well-isolating barrier, as for example a pinched-off connection or a sealed foil. In the case of ALPHATRAP, no permanent barrier can be used to effectively transmit HCI of several $10 \mathrm{keV}$ kinetic energy from the ion source to the Penning trap while blocking neutral gas particles. Rather, the cryogenic valve (see Sect. 4.2.2) developed and installed at ALPHATRAP prevents a continuous stream of background gas particles from the room temperature beamline into the cryogenic trap chamber. However, to also prevent excessive particle flux during the opening periods of the valve, the beamline itself has to provide a 
background gas pressure in the order of $10^{-11}$ mbar at room temperature. To match these requirements on the background gas pressure in the vertical section of the AlPhatrap beamline, see Figure 13, the following design and construction strategy has been pursued:

- All vacuum components have to be in situ bakeable to $300^{\circ} \mathrm{C}$. This reduces the suitable materials to UHV compatible metals, ceramics and synthetics with low vapor pressure at elevated temperatures.

- The major background gas partial pressure in the UHV regime results from hydrogen outgassing of stainless steel components. This can be reduced by vacuum firing $\left(950^{\circ} \mathrm{C}\right.$ at $10^{-5}$ mbar or better for at least $\left.1.5 \mathrm{~h}\right)$ after the final machining, which also decreases the permeability of e.g. welds of stainless steel which becomes necessary in the vicinity of the superconducting magnet. Usually, it is recommended to only use conflat (CF) flange components made from material 1.4429ESU for vacuum firing to preserve the stability of the sealing forming knife. In-vacuum ion optical components were made mainly from 1.4429ESU or 1.4435 stainless steel and vacuum fired as well. Insulators are made from high-purity alumina or Macor. Further materials used in small quantities are phosphor bronze, aluminum and beryllium copper and have been vacuum fired before installation at suitable temperatures. After this treatment, the surface of the components is basically free from organic components, reducing the risk of contaminating the vacuum.

- Prior to the final installation all parts are cleaned in acetone and isopropanol, this also accounts for vacuum components that could not be vacuum-fired after final machining such as membrane bellows or parts with glass- or ceramic-metal joints.

- In-vacuum cabling has been performed with blank copper wire supported by alumina shells at critical positions to avoid the usage of polyimide-coated wires, which are known for their moisture sorption.

To evacuate the beamline, several turbomolecular pumps (TMP), ion-getter pumps (IGP) and non-evaporative getter (NEG) pumps are used, as illustarted in Figure 14. To eliminate the risk of vacuum contamination with organic compounds due to outgassing and backflow in case of TMP failure, all TMPs used (except TMP HC-EBIT) are fully magnetically levitated (Edwards STP603) and therefore lubricant free. The pre-vacuum is generated with oil-free scroll pumps (Edwards nXDS series). To improve the pre-vacuum and therefore also the overall effective compression ratio for $\mathrm{H}_{2}$, a $80 \mathrm{l} / \mathrm{s}$ TMP is installed in the pre-vacuum system (Oerlikon Leybold Turbovac SL80). In the vertical beamline, where a low background gas pressure is most critical due to the close proximity to the Penning trap and the clear line of sight into the center of the trap while the cryogenic valve is open, an ion getter pump (IGP, Agilent VacIon Plus 500 Starcell), a non-evaporative getter pump (NEG 1, SAES Capacitorr-D 2000/150 MK5) and an IGP-NEG combination (NEG 2, SAES NEXTorr D 2000-10) are installed additionally. After evacuation for several weeks with only TMPs, the background gas pressure settled at around $10^{-10} \mathrm{mbar}$ (air equivalent) or better in most parts of the beamline, measured with several Penning gauges (Pfeiffer Vacuum IKR270) along the beamline. This has been achieved without an in situ bakeout and activation and/or conditioning of the IGP and NEG pumps and has been considered as sufficient so far. It is believed that the background gas pressure will be further reduced by an in situ bakeout and activation of these pumps, however this has not yet been performed.

For ion beam and pulse diagnosis, several microchannel plate (MCP) detectors (Photonis USA APD 2 PS 18/12/10/5 I 40:1 P43 or El-Mul Technologies C033VP43) 


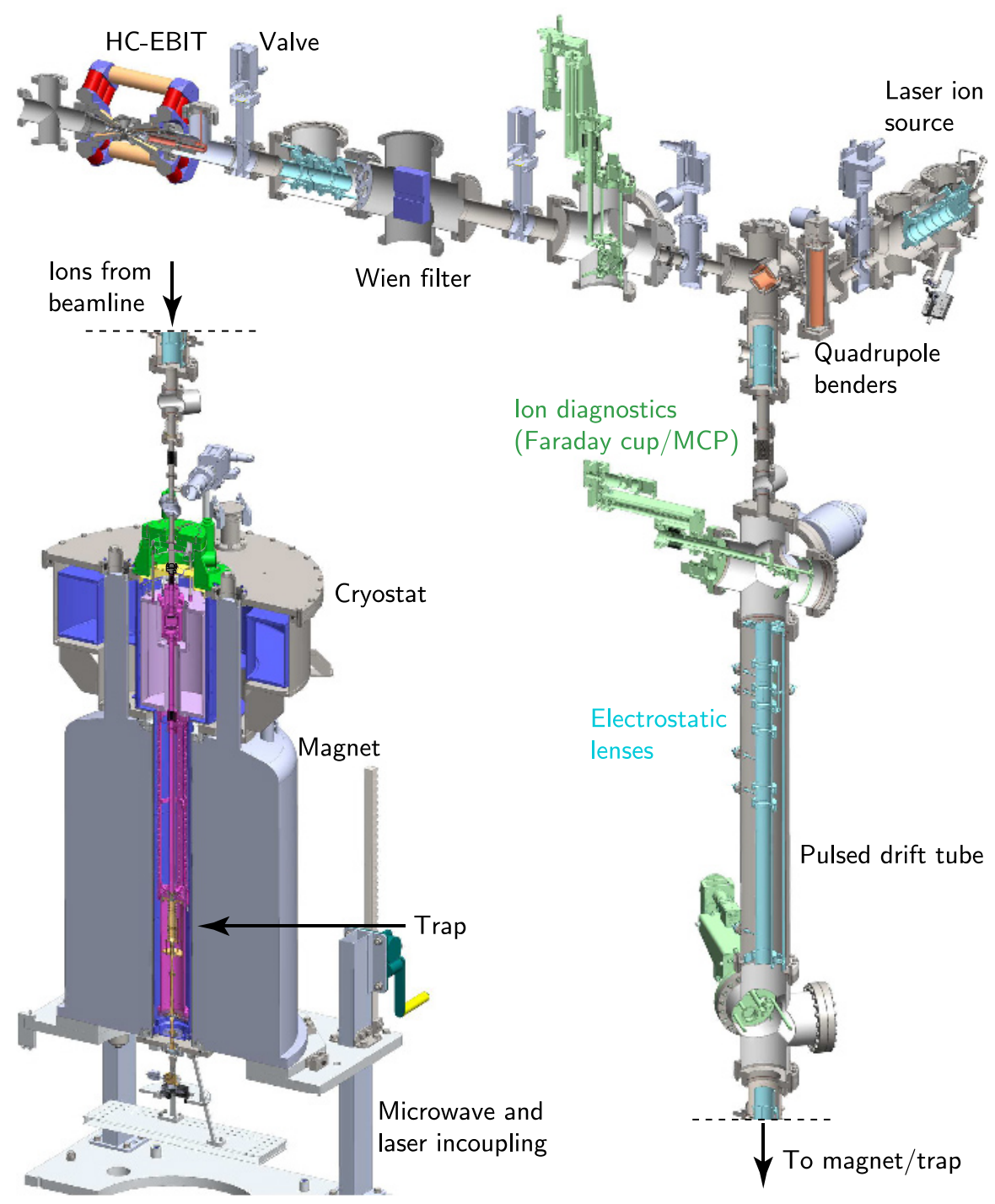

Fig. 13. Cut-open view of the Alphatrap electrostatic beamline. Ions are arriving in short pulses from either the room-temperature HC-EBIT, the superconducting HD-EBIT (from the right) or the Laser ablation source at transfer potentials of a few $\mathrm{keV} \times \mathrm{q}$ in the ground floor. Two electrostatic quadrupole benders deflect the beam first into the plane of the vertical beamline if necessary and then down into the basement, where the main laboratory which houses the magnet is located. A pulsed drift-tube allows us to remove most of the kinetic transfer energy. The ions are exiting the pulsed drift tube with about $100 \mathrm{eV} \times \mathrm{q}$. Electrostatic lenses and steerers allow refocussing the beam into the ALPHATRAP cryostat and magnet. Here, they pass the cryogenic vacuum valve and are eventually recaptured in the trap tower. The arrival of the bunches can be monitored using the cryogenic faraday-cup detector (see Fig. 24).

are installed along the beamline, while all except MCP2 are mounted on a motion feedthrough to retract them from the ion optical axis. The detectors are equipped with a phosphor screen and an in-vacuum highly polished aluminum disc mounted $45^{\circ}$ tilted to the normal of the phosphor screen and a triggerable CCD camera (Allied 


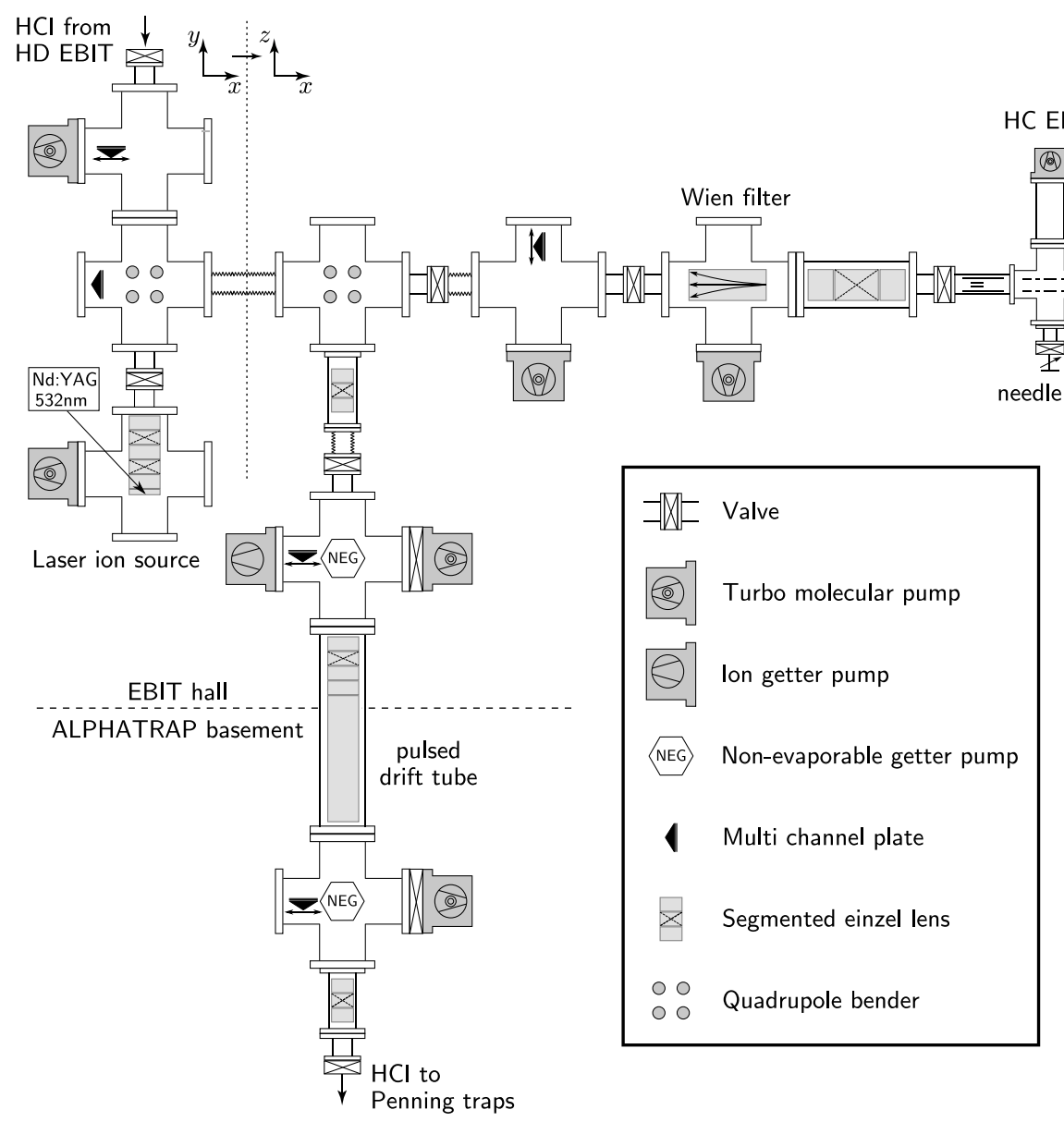

Fig. 14. Schematic overview of the AlPhatrap room-temperature beamline. Ions are produced in either the HD-EBIT, the HC-EBIT or the laser ion source (LIS) and transferred via various ion optics from the EBIT hall level to the Penning-trap setup in the basement. The positions of turbomolecular pumps (TMP), an ion getter pump (IGP), non-evaporative getter (NEG) pumps, microchannel plate (MCP) detectors and quadrupole benders (QPB) are indicated as well. For further details see text.

Vision Prosilica GC655) outside the vacuum chamber to record the position of the ion beam. In addition, the electronic signal of the MCP following an ion impact is amplified (FAST Comtec TA2000B-3 or Ortec VT120A) and recorded with a fast multi scaler (Stanford Research Systems SR430 or FAST ComTec MCS6A2) to acquire the pulse shape and timing information.

To inject ions into the Penning-trap system inside the superconducting magnet (see cut-open view in Fig. 13), the ions have to pass an $8 \mathrm{~mm}$ inner diameter bellow tube of about $29 \mathrm{~mm}$ length before they are entering the magnetic field gradient. Further downstream in the high-field region close to the trap entrance, a tubular aperture of $3 \mathrm{~mm}$ diameter and $5.9 \mathrm{~mm}$ length is installed and acts as a pumping barrier as well as Faraday cup for ions on large radial positions. To pass the bellow and the aperture, the ion pulses have to be steered and focused precisely, which is accomplished by a combination of three $x-y$ segmented einzel lenses in the vertical section of the beamline, see Figure 13. Ion pulses are typically extracted from EBIT 
electrodes biased to potentials of several kilovolts with respect to ground. To capture the ions at almost rest in the Penning trap, identical electric potentials would have to be applied and switched in the electrode structure of the Penning trap. Since this is technically very challenging, the ions are slowed down via a pulsed drift-tube in the vertical section of the room temperature beamline to a low kinetic energy of about $100 \mathrm{eV} \times \mathrm{q}$, such that the final deceleration in the Penning-trap structure only requires voltages of about $100 \mathrm{~V}$. To estimate the transfer efficiency, the ion beam current from the HC-EBIT has been measured directly by using the Faraday cup after the Wien filter. An ion count of about $14500{ }^{16} \mathrm{O}^{5+}$ can be extracted by pulsing the center drift tube of the HC-EBIT. After lowering the energy of the ions to around $100 \mathrm{eV} \times \mathrm{q}$, about 410 ions can be detected in the croygenic Faraday cup at the bottom of the Penning trap. This results in a transfer efficiency of approximately $3 \%$.

\subsection{The Alphatrap ion sources}

The Alphatrap setup contains several ion sources to produce heavy HCI as the Heidelberg EBIT, medium and low mass HCI as the HC-EBIT and singly charged ions as the laser ablation ion source. These ion sources will be described in detail in the following.

\subsubsection{Heidelberg EBIT}

The Heidelberg EBIT will be the main ion source for AlPhatrap. It is designed to allow the electron gun to generate a beam with up to $150 \mathrm{keV}$ energy and a current in excess of $500 \mathrm{~mA}$. Such parameters also require suitable shielding for X-rays. The EBIT main magnetic field is generated by a superconducting magnet, which reaches up to $9 \mathrm{~T}$ in the central beam compression region. Here, beam densities in the order of $15000 \frac{\mathrm{A}}{\mathrm{cm}^{2}}$ are achieved. To minimise charge-exchange processes with the HCI, the EBIT electrode structures are cooled to liquid helium temperature, which enables very good vacuum conditions. Combined with the injection of a light cooler gas, which is expelled from the trapping region after gaining energy from collisions with the heavy HCI and thus cools them, the EBIT in principle can produce even the highest charge states of heavy ions, including hydrogenlike lead ${ }^{208} \mathrm{~Pb}^{81+}$. Currently, the EBIT is being re-commissioned to achieve the design electron energies, but already the current performance of up to $70 \mathrm{keV}$ gives access to a multitude of interesting candidates for $g$-factor measurements in a previously inaccessible regime, such as lithiumlike bismuth ${ }^{209} \mathrm{Bi}^{80+}$ and hydrogenlike ions up to ${ }^{132} \mathrm{Xe}^{53+}$. The high transfer efficiencies achieved with the AlPHATRAP beamline and the virtually infinite storage times in the cryogenic Penning trap allow to work even with very low production yields. After the charge breeding process, which can take up to minutes depending on the addressed charge state, the HCI are ejected in bunches from the EBIT. The transfer energy is adjustable, but typically of the order $10 \mathrm{keV} \times \mathrm{q}$. The ions of interest can be selected by a dipole magnet. After focussing and steering elements, the beam passes straight through a selector magnet and enters the ALPHATRAP beamline, from where it is finally guided into the trap section. In AlPHATRAP, the ion bunch is kept in the storage trap, from where HCI can be extracted at wish. This way, the EBIT can be used for other experiments or even switched off after the trap has been loaded.

\subsubsection{Heidelberg compact electron beam ion trap}

The Heidelberg compact electron beam ion trap (HC-EBIT) is a room-temperature, permanent magnet EBIT developed at the Max Planck Institute for Nuclear Physics. 
AlPhatrap uses a prototype while several follow-up versions are already in use at other experiments [59].

The magnetic structure consists of eight arrays of each six NdFeB cylinder magnets ( $45 \mathrm{~mm}$ diameter, $30 \mathrm{~mm}$ height). Soft iron is used as a yoke to concentrate the magnetic flux and to achieve about $0.74 \mathrm{~T}$ in the $19 \mathrm{~mm}$ wide magnetic gap in the center of the structure. Later versions with nine cylinder magnets per array achieve even $0.87 \mathrm{~T}$ in the center. Flux return rods on the outside close the magnetic circuit. Regions of almost zero magnetic field are formed on the axis at about $90 \mathrm{~mm}$ distance from the center, ideally used as electron emitting and collecting locations. A $3.4 \mathrm{~mm}$-diameter thermionic barium impregnated tungsten-dispenser cathode with a concave spherical radius of $8.2 \mathrm{~mm}$ is used in a Pierce-type electron gun incorporating a focusing and anode electrode to control the electric field strength and shape at the cathode. Stable and constant electron emission currents of several milliampere can be achieved at vacuum levels on the order of $1 \times 10^{-9}$ mbar. The electron collector on the opposite side of the magnetic center consists of a copper tube held at ground potential and a focusing electrode at negative potential to focus the ions. The electron transmission, i.e., the ratio of the emitted electron current from the cathode and the electron current on the collector is about $93 \%$. The electron beam is compressed by the high magnetic field in the center of the structure, yielding there the highest ionization rates. Here, an electrode structure is located to capture and store the ions. The cathode is typically at potential of $-1 \mathrm{kV}$ to $-1.5 \mathrm{kV}$ with respect to ground while the inner electrodes are at potentials of about $+2 \mathrm{kV}$, resulting in electron energies of up to $3.5 \mathrm{keV}$ in the center of the structure.

In the case of the HC-EBIT, the electrostatic structure consists of five cylindrical electrodes where the innermost electrode is held at a lower positive potential than the outer electrodes to capture ions axially. The radial confinement is achieved by the Lorentz force due to the magnetic field and the electric field due to the space charge potential of the electron beam and the electrodes. Gas-phase atoms such as noble gasses, e.g. argon or xenon, are introduced by means of a needle valve. For optimal ionization, the gas pressure ranges from $1 \times 10^{-9}$ mbar to about $3 \times 10^{-9}$ mbar, measured above the central region of the structure. HCI up to ${ }^{40} \mathrm{Ar}^{16+}$ and ${ }^{129} \mathrm{Xe}^{37+}$ have been produced in a pulsed ejection mode [59]. Ions of different specific charge are spatially separated with a Wien filter, and only the species of interest is transferred on the optical axis of the system to the Penning trap, while all other species are lost in the transfer process.

\subsubsection{In-trap EBIS}

An in-trap Electron Beam Ion Source (EBIS) [68] has been used for the first commissioning stages of our experiment. This ion source consists of a field-emission point (FEP), an acceleration electrode, a carbon target and a set of electrodes which are used to reflect the electron beam at the capture-trap section as shown in Figure 15.

The electrons are extracted from the tip of the field emission point by applying a voltage difference between the cathode and the acceleration electrodes. Since the electron beam is reflected several times the current density continuously increases until the space charge of the electrons cause the beam to hit the target, which has a $0.8 \mathrm{~mm}$ hole. As a result, a number of atoms are evaporated from the target's surface and fills the trap volume. Due to further collisions with the electron beam these atoms can be ionised and trapped in the precision-trap region (see Fig. 15 and Sect. 4.5). The resulting ion cloud consists of several species with ions in different charge-to-mass ratios.

The in-trap EBIS allowed for testing different parts of our complicated setup individually. By calibrating the trap tower using carbon ions, from the in-trap source, we 


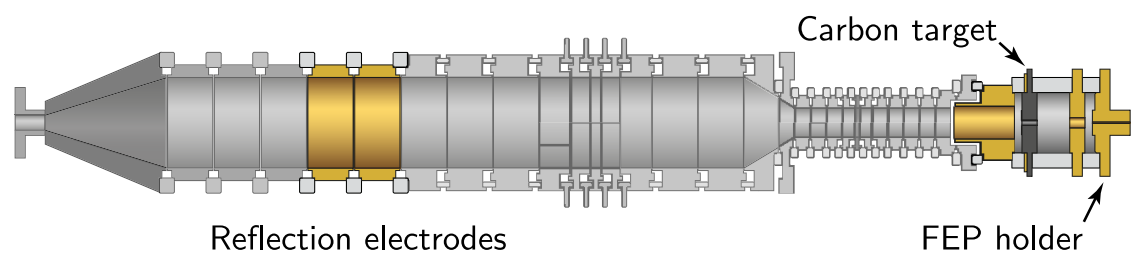

Fig. 15. In-trap Electron Beam Ion Source that was temporarily attached at the bottom of the trap tower. A field emission point (FEP) emits an electron beam that is reflected within the trap via the reflection electrodes, before the electron beam hits the target and sputters atoms. Those atoms are subsequently ionized and can be captured in one of the traps. The rest of the trap tower is shown here as transparent. For details see text.

were able to separate the optimization of the external ion injection and the ion trapping and detection, an important advantage. Eventually, the electron gun has been removed from the trap tower to allow the injection of miliimeter and laser excitations.

\subsubsection{Laser ion source}

In our laser ion source (LIS) [69], atoms are ablated or vaporised from solid target materials. This is done using a high intensity laser pulse, produced by a frequency doubled Nd:YAG laser system (Litron Nano S) with a maximum pulse energy of $35 \mathrm{~mJ}$ and a pulse length of $6 \mathrm{~ns}$ to $8 \mathrm{~ns}$ at $532 \mathrm{~nm}$. If the applied power density exceeds $1 \times 10^{8} \mathrm{~W} / \mathrm{cm}^{2}$, a plasma can be produced by the same laser pulse. Such conditions are achieved by focusing the laser down to a spot diameter of less than $250 \mu \mathrm{m}$. The higher thermal velocity of the electrons compared to the ions leads to a faster diffusion of the electrons as well as a higher probability of them hitting surrounding electrodes. This results in an overall net of positively charged ions, which can be extracted by applying a constant electric field.

The LIS consists of two double vacuum crosses to house the ablation chamber, the acceleration stages, and a set of three einzel lenses as shown in Figure 16. The laser is introduced through a $45^{\circ}$ tilted view-port. The acceleration stage is split into three parts which allows the selection of the distance of the time-focal-point according to [70]. The set of three einzel lenses, including two 4-way split electrodes in between facilitates beam focusing and steering, as well as a changing of the lateral position of the beam.

The motorised $x-y$ translation stage can be used to manipulate the target position. By moving the target to a specific position, a hole in the target holder will allow the laser to pass through the setup and to be monitored and adjusted through the upper view-port. With different materials mounted on the target holder this also enables a selection of the target material giving access to different ion species. This has been successfully tested using carbon, aluminum and aluminum-beryllium compound targets, the latter being used for the production of beryllium ions. The linear feedthrough ( $z$ translation) allows the precise positioning of the target close to the acceleration stage.

Measurements of the ion beam current showed a production of up to $2.6 \times 10^{7}$ ${ }^{9} \mathrm{Be}^{+}$ions per laser pulse. This is expected to allow a sufficient amount of beryllium ions to be transferred and trapped to facilitate laser cooling.

\subsection{Trap tower}

The Alphatrap trap tower consists of a double Penning-trap system and a set of capture electrodes (see Fig. 17). The capture trap is used for capturing externally 


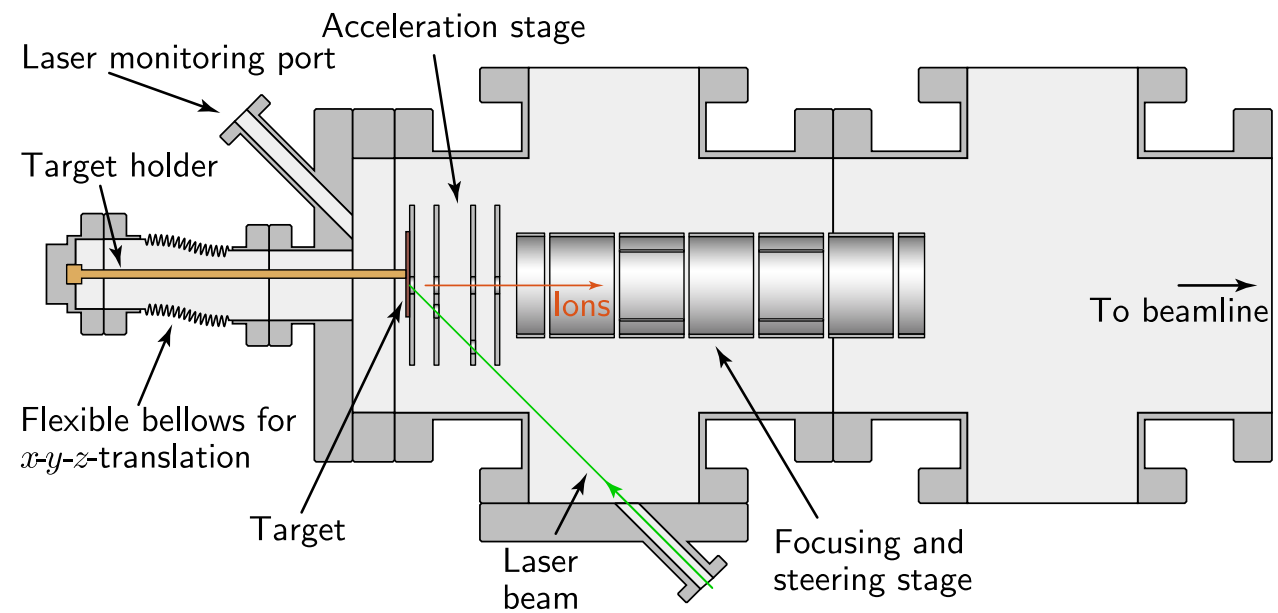

Fig. 16. Simplified schematic of the laser ion source (LIS): A frequency doubled Nd:YAG laser at $532 \mathrm{~nm}$ enters the vacuum chamber with $45^{\circ}$ angle. The laser hits the target material, vaporises it and ignites a plasma. From this, ions are extracted by applying a high voltage to the acceleration electrodes. The extracted ions are then steered into the beamline and guided towards the trap.

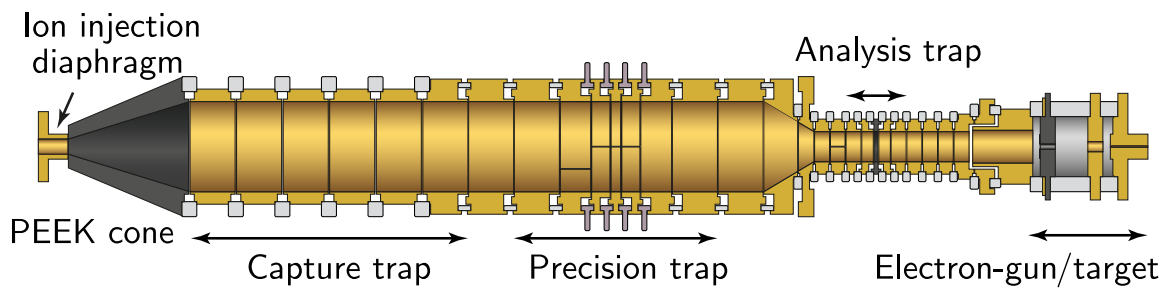

Fig. 17. The Alphatrap trap tower. The precision trap is used for high-precision spectroscopy and the analysis trap is used for the spin-state determination via the continuous Stern-Gerlach effect. The ions enter via the ion injection diaphragm before they are captured within the capture trap. For commissioning purposes, an electron gun was implemented (Sect. 4.4.3) for in situ ion production.

injected ions by fast switching of the potential of this set of electrodes. The double Penning-trap system involves the so called "precision trap" and "analysis trap", which are the tools necessary for the $g$-factor determination via the double-trap method (see Sect. 3.7). During the first commissioning stage of our experiment, an electron gun attached to the bottom of the trap tower was used. It can be seen in Figure 17, and is described in Section 4.4.3.

The capture trap, the precision trap and the analysis trap are separated by a series of transport electrodes that allow adiabatic ion transport from one trap to another. The externally injected ions have to travel through the ion injection diaphragm with $3 \mathrm{~mm}$ inner diameter that was planned to be used also as a Faraday Cup during the first attempts of external ion injection. Between the diaphragm and the capture trap a microwave damping cone made from carbon nanotube filled peek (TECAPEEK ELS nanoblack [71]) is installed, which has a specific volume resistance within the range of $10^{3} \Omega \mathrm{cm}$ to $10^{5} \Omega \mathrm{cm}$. Every electrode is isolated from its neighboring electrodes by sapphire or quartz insulators. ${ }^{2}$

\footnotetext{
${ }^{2}$ Both the sapphire and the quartz-glass insulators were manufactured with tolerances down to $3 \mu \mathrm{m}$ for the critical surfaces.
} 


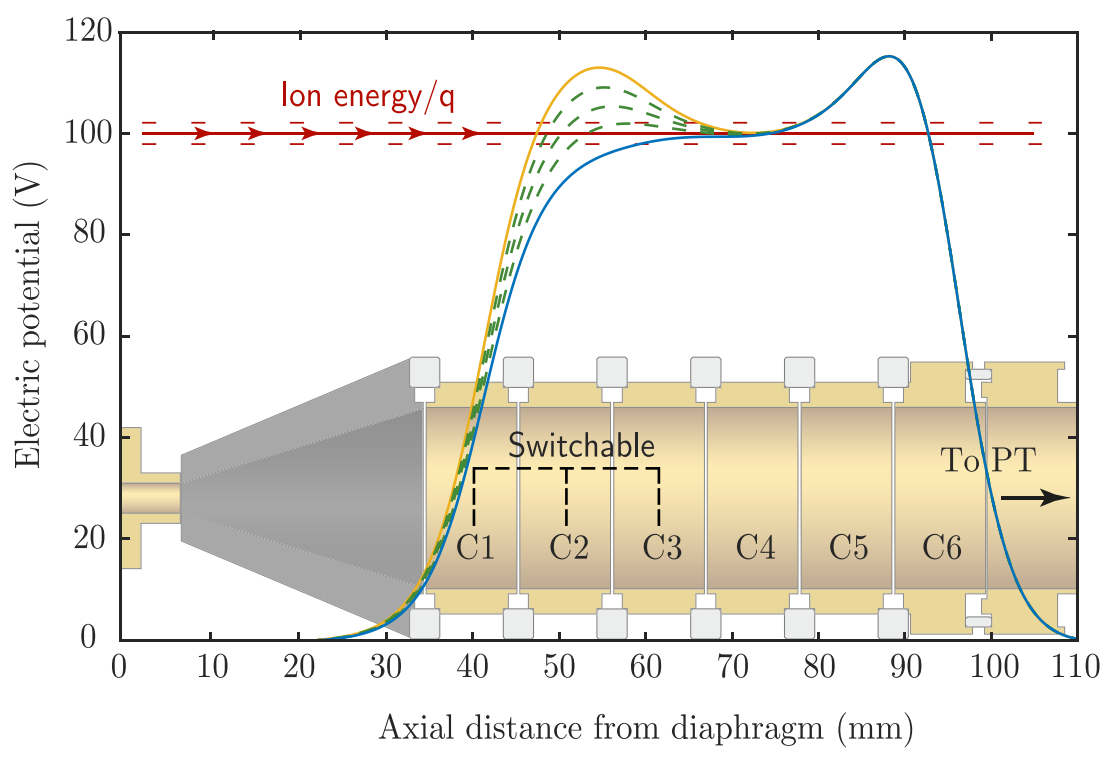

Fig. 18. Illustration of a typical potential curve applied to the capture electrodes during the injection of ions with an average energy of $100 \mathrm{eV} \times \mathrm{q}$. The capture section consists of six individually controlled electrodes of which the first three are switchable within $1 \mu \mathrm{s}$. After slowing down (by the blue curve) the ion bunch is captured in a potential well (blue and yellow curve). The switching process is illustrated by the green dashed curves.

\subsubsection{Capture trap}

The HCI from the different ion sources are all decelerated by the pulsed drift tube and focused by the electronic optical lens and guided towards the Penning trap. After passing through the 3-mm diaphragm, the ion bunch enters the capture section which consists of six individually controlled electrodes. To capture the ions, the first three electrodes of the capture trap can be switched by a maximum of $\pm 200 \mathrm{~V}$ within $1 \mu \mathrm{s}$. By adjusting the capture potential according to the energy distribution of the ion bunch the capture efficiency can be optimised. A typical potential configuration is shown in Figure 18. In a first step the ion bunch is slowed down and spatially compressed. Subsequently, it is captured in a potential well by applying a fast switching pulse to the C1-C3 electrodes when the bunch is at its motional reversal point. Thus, the remaining axial energy is mainly determined by the initial energy dispersion of the ion bunch. For HCI injected from the HC-EBIT the axial energy dispersion is typically on the order of $5 \mathrm{eV} \times \mathrm{q}$ to $10 \mathrm{eV} \times \mathrm{q}$. After a successful injection the capture section is used as a battery buffered reservoir. From this reservoir, ions can be extracted by applying suitable voltages to the center electrode of this trap well, which splits the trap and the cloud in two parts. This allows for continuous operation largely independently of the external ion sources.

\subsubsection{Precision trap}

In order to allow for high-precision experiments with both low and highly charged ions, the precision trap has to meet a number of special requirements. For this reason, the Alphatrap precision trap is larger than predecessor high-precision Penning traps and features an exceptionally harmonic electrostatic potential. In Figure 19 
the precision-trap electrodes are depicted. The trap features some non-standard characteristics.

\section{(1) Large radius}

All electrodes of the precision trap have a radius of $9 \mathrm{~mm}$ predominantly to reduce systematic uncertainties that are caused by the image charge shift $[51,72]$ of the ion's free cyclotron frequency, which scales with $\propto \frac{1}{\rho^{3}}$ :

$$
\frac{\delta \nu_{\mathrm{c}}}{\nu_{\mathrm{c}}} \propto \frac{m}{8 \pi \epsilon_{0} \rho^{3} B_{0}^{2}}
$$

with $m$ being the mass of the ion, $\rho$ the trap radius and $B_{0}$ the magnetic field strength. Since the relative image charge shift scales linearly with the ion's mass, it would be dominant for heavy ions such as ${ }^{208} \mathrm{~Pb}^{81+}$. With the optimised trap geometry with $\rho=9 \mathrm{~mm}$, the image charge shift is reduced by more than an order of magnitude compared to predecessor experiments using only $\rho=3.5 \mathrm{~mm}$. Additionally, the effect of patch potentials on the electrode surfaces on the ion's motion are also reduced with a large trap radius. Such patch potentials can occur due to variations of the work function of the gold layer or accumulation of charges on the electrodes during ion loading.

\section{(2) 7-electrode configuration}

Higher electric field harmonicity allows excitation to larger amplitudes without the effect of systematic shifts that would arise otherwise, an advantageous configuration for detecting even low charged ions. Cylindrical traps can be made harmonic by suitable electrode geometries or by proper tuning of the applied voltages (see Sect. 5.2) in order to correct for the unavoidable deviation from the ideal trap case. Most cylindrical Penning traps are composed of a stack of 5 electrodes, namely a ring electrode, two correction electrodes positioned symmetrically around the ring electrode on the axial direction and a set of endcap electrodes that are typically grounded. Introducing an extra set of correction electrodes (see Fig. 19), allows for compensating of higher order field components via two degrees of freedom, leading to much better harmonicity. That way excitations of the ion's motion to larger radii are enabled, an essential feature for high-precision measurement techniques like PnA [42,51].

To prevent undesired effects due to paramagnetic oxygen contaminations, which would lead to sizeable fluctuations of the magnetic field at cryogenic temperatures, all electrodes are made out of Oxygen Free High Conductivity (OFHC) copper and have been machined with a tolerance of $10 \mu \mathrm{m}$. After manufacturing, in order to avoid oxidation, all electrodes were subjected to gold-plating, where a uniform layer of $10 \mu \mathrm{m}$ of gold was deposited on the electrodes' surfaces via galvanic deposition. A $2 \mu \mathrm{m}$ silver layer acts as a diffusion barrier between copper and gold.

The Alphatrap precision trap consists of a ring electrode, two sets of correction electrodes and endcap electrodes (see Fig. 19). The ideally very long endcap electrodes have been split in shorter sections. That way the ion can be adiabatically transported to and from the precision trap. The dimensions of our precision trap, which is designed similarly to the precision trap of the Light Ion Trap (LIONTRAP) [41,73], a high-precision mass spectrometer for light ions in Mainz, are listed in Table 1 and have been calculated analytically during the optimisation process and independently verified numerically as well as analytically [34]. In this 7-electrode configuration, five parameters can vary towards the optimum geometry for the highest 


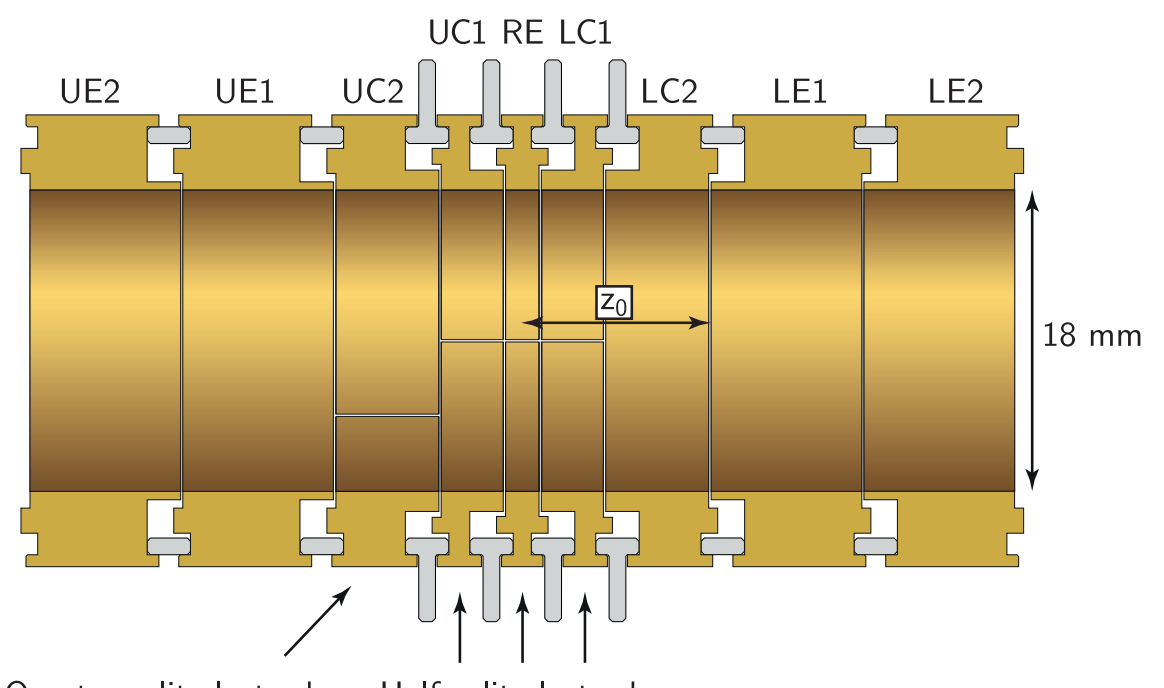

Quarter-split electrode Half-split electrodes

Fig. 19. The precision trap of AlphatRap is shown. It consists of a ring electrode (RE), two sets of correction electrodes - upper correction 1 (UC1), lower correction 1 (LC1) and upper correction 2 (UC2), lower correction 2 (LC2) - and four endcap electrodes (UE1, LE1, UE2 and LE2). The sapphire and glass insulators are shown in grey. The ring electrode as well as the upper and lower correction electrode 1 are half-split while the upper correction electrode 2 is quarter split. For details see text.

Table 1. Precision-trap electrode's optimum dimensions. The manufacturing tolerance was $10 \mu \mathrm{m}$. This seven-electrode trap consists of a ring electrode, two sets of correction electrodes and two sets of endcap electrodes rather than one set of double-in-length endcap electrodes. Functionally, the endcaps (together with the rest of the trap tower), act together to define the ground potential reference. For details see text.

\begin{tabular}{lll}
\hline Electrode & Abbreviation & Length $(\mathrm{mm})$ \\
\hline Ring & $\mathrm{RE}$ & 1.993 \\
Correction 1 & $L C_{1} / U C_{1}$ & 3.705 \\
Correction 2 & $L C_{2} / U C_{2}$ & 6.146 \\
Endcap & $L E_{1,2} / U E_{1,2}$ & 8.996 \\
\hline
\end{tabular}

electric field harmonicity. For a fixed trap radius and gap between adjacent electrodes, these parameters are the length of the ring and of the correction 1 and correction 2 electrodes as well as the voltages applied to the correction electrodes $\left(l_{\mathrm{r}}, l_{\mathrm{c} 1}, l_{\mathrm{c} 2}, V_{\mathrm{c} 1}, V_{\mathrm{c} 2}\right)$. The potential in the gap between electrodes has been modeled either as a linear potential or as a 5 th order polynomial [74,75]. It should be noted that in our calculations we found good agreement between the calculations mentioned above.

Assuming an empty trap and cylindrical as well as axial mirror symmetry with respect to the trap centre, the electrostatic potential inside the trap follows equation (8) where the $C_{n}$ coefficients are dimensionless expansion parameters, $V_{0}$ is the voltage difference between the ring and the endcap electrodes, $d=$ $\sqrt{\frac{1}{2}\left(z_{0}^{2}+\frac{\rho^{2}}{2}\right)}=9.156 \mathrm{~mm}$ is a characteristic length of the trap and $z_{0}$ is defined as $z_{0}=l_{\mathrm{r}} / 2+l_{\mathrm{g}}+l_{\mathrm{c} 1}+l_{\mathrm{g}}+l_{\mathrm{c} 2}+l_{\mathrm{g}}$. The optimisation parameters $\left(l_{\mathrm{r}}, l_{\mathrm{c} 1}, l_{\mathrm{c} 2}, V_{\mathrm{c} 1}, V_{\mathrm{c} 2}\right)$ 
have been chosen such that $C_{4}=C_{6}=C_{8}=C_{10}=0$ as well as $D_{2}^{\text {comb }}=0$ where

$$
\begin{gathered}
C_{2, \mathrm{eff}}=C_{2}+D_{2}^{\mathrm{comb}} \\
D_{2}^{\mathrm{comb}} \equiv D_{21} \cdot T R_{1}+D_{22} \cdot T R_{2},
\end{gathered}
$$

and

$$
T R_{1,2}=\frac{V_{c 1,2}}{V_{r}}
$$

Here, the so-called tuning ratios $T R_{1}$ and $T R_{2}$ are defined which are the ratios between the voltages applied to the respective correction electrode and the voltage applied to the ring electrode. By optimising both tuning ratios in situ, it is possible to completely null at least $C_{4}$ and $C_{6}$ simultaneously even in the presence of unavoidable machining imperfections. In this case the robustness of our trap design typically causes $C_{8}$ to remain small as well. The remaining anharmonicities lead to frequency shifts that are typically negligible even for high-precision measurements.

To be able to practically operate the trap, it is important that the orthogonality criterion is fulfilled. That is, the axial frequency $\nu_{z} \propto \sqrt{C_{2}}$ (see Eq. (9)), remains unaffected while changing the applied tuning ratio. Since double orthogonality $\left(D_{21}=\right.$ $D_{22}=0$ ) is not possible in this kind of configuration, the trap is orthogonalised by imposing the "combined orthogonality" criterion of equation (31). On the inner cylinder surface all electrodes have a distance of $l_{g}=140 \mu \mathrm{m}$ from their adjacent electrodes, a distance maintained by means of sapphire or quartz-glass ring insulators. As can be seen in Figure 19, the insulators between the ring and correction electrodes are T-shaped. They are made out of quartz glass and have been machined to at least the same precision as the electrodes. Since the shrinking coefficient of sapphire is significantly smaller than the one of copper, the electrodes shrink onto the insulators in a controlled fashion. For this reason the T-shaped design has been employed, which allows for a well-defined final position of the electrodes when cooling down from room temperature to $4 \mathrm{~K}$. The same distance of $140 \mu \mathrm{m}$ separates the segmented electrodes. The ring electrode as well as the set of first correction electrodes are half split allowing dipole excitation for driving the modes and quadrupole excitation that is used for coupling the radial modes to the axial one via sideband coupling (see Sect. 3.4). The upper correction electrode 2 is quarter split permitting radial mode coupling. All split electrodes in the precision trap are insulated via sapphire plates, which are invisible in Figures 17 or 19.

\section{(3) Magnetic field homogeneity}

Similarly to the electric field, the magnetic field can be expanded in a series of Legendre polynomials. When considering only the axial component of the magnetic field the series expansion is further simplified:

$$
B_{z}(z)=\sum_{i=0}^{\infty} B_{i} z^{i}
$$

The shift due to the leading order correction, the quadratic magnetic bottle $\sim B_{2} z^{2}$, has been derived in [36] and reads: 
Table 2. Exemplary expected axial frequency jumps induced by a spinflip in the analysis trap of AlPhATRAP with $B_{2}=44.35(84) \mathrm{kT} / \mathrm{m}^{2}$ (see Sect. 5.4 ), $B_{0} \simeq 3.8 \mathrm{~T}$ and $\nu_{z} \simeq 334 \mathrm{kHz}$.

\begin{tabular}{|c|c|c|c|c|c|c|}
\hline & ${ }^{12} \mathrm{C}^{5+}$ & ${ }^{28} \mathrm{Si}^{13+}$ & ${ }^{40} \mathrm{Ar}^{13+}$ & ${ }^{132} \mathrm{Xe}^{53+}$ & ${ }^{208} \mathrm{~Pb}^{81+}$ & ${ }^{208} \mathrm{~Pb}^{77+}$ \\
\hline$\Delta \nu_{z}$ & $3.1 \mathrm{~Hz}$ & $1.3 \mathrm{~Hz}$ & $312 \mathrm{mHz}$ & $269 \mathrm{mHz}$ & $156 \mathrm{mHz}$ & $54 \mathrm{mHz}$ \\
\hline & $\left.\begin{array}{l}/ \nu_{+} \\
/ \nu_{z} \\
/ \nu_{-}\end{array}\right)$ & $=\frac{L}{B_{0} m(}$ & $\frac{}{\left.\nu_{z}\right)^{2}}$ & $\begin{array}{c}\left(\nu_{z} / \nu_{+}\right)^{2} \\
1 \\
2\end{array}$ & $\left.\begin{array}{cc}1 & 2 \\
0 & -1 \\
-1 & -2\end{array}\right)$ & $\left(\begin{array}{c}E_{+} \\
E_{z} \\
E_{-}\end{array}\right)$ \\
\hline
\end{tabular}

For high-precision determinations of the ion's free cyclotron frequency, not only electric field homogeneity is necessary but also homogeneity of the magnetic field is essential. Due to the shimming procedure, the magnetic field of our superconducting magnet is very homogenous without the trap tower setup. When the double-trap setup (see Sect. 3.7) is inserted inside the bore of the magnet, the dominant magnetic field inhomogeneity contribution in the precision-trap region is the residual $B_{2}$ due to the magnetic bottle configuration. For this reason, a ring that is also made out of the same ferromagnetic material VACOFLUX50 [43] was designed and positioned appropriately so that it compensates for magnetic field inhomogeneities notably, about one order of magnitude. The resulting $B_{2}$ in the precision trap was measured to be $B_{2}=0.0643(32) \mathrm{T} / \mathrm{m}^{2}$ and the linear field gradient of our setup is $B_{1}=2.638(24) \mathrm{mT} / \mathrm{m}$.

\subsubsection{Analysis trap}

The analysis trap is dedicated to the determination of the ion's spin state via the continuous Stern-Gerlach effect [31] (see Sect. 3.6). There, in contrast to the precision trap, a very strong magnetic inhomogeneity is required in order to allow for detectable axial frequency jumps caused by spin flips. This trap differs from the precision trap in a few important aspects: it acquires a ferromagnetic ring electrode, it is smaller in dimensions and it is a 5 -electrode trap.

\section{(1) Magnetic bottle}

The ring electrode of this trap is made out of VACOFLUX50 [43], which has a very high saturation magnetisation corresponding to about $2.35 \mathrm{~T}$ and can locally generate the strong magnetic bottle configuration that is necessary for the spin flip detection (for details see Sect. 3.6). The material choice and the geometry have been optimised so that a magnetic bottle of strength $B_{2} \approx 44 \mathrm{kT} / \mathrm{m}^{2}$ is achieved. Inducing a change in the spin projection translates into a jump of the axial frequency of the ion in the analysis trap according to equation (25). The expected frequency shifts for some light and heavy HCI in our setup are given in Table 2. These depend primarily on the strength of the magnetic bottle and the mass of the ion.

\section{(2) 5-electrode configuration}

The analysis trap, shown in Figure 20, is much smaller than the precision trap. With a radius of $\rho_{\mathrm{AT}}=3 \mathrm{~mm}$ the necessary strong magnetic bottle configuration is enabled. The lower limit for the radius of the analysis trap was set by the spin transition frequency of boronlike lead ${ }^{208} \mathrm{~Pb}^{77+}$, as a smaller radius would not support 


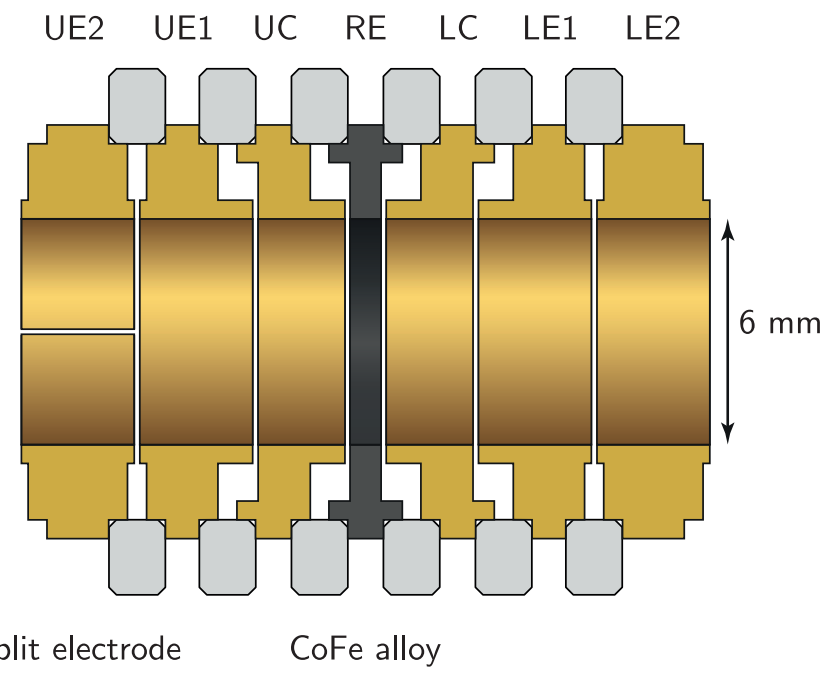

Fig. 20. The analysis trap of Alphatrap consists of a ring electrode (RE), a set of correction electrodes (upper correction (UC) and lower correction electrodes (LC)) and two sets of endcap electrodes (upper endcap 1 and 2 (UE1 and UE2) and lower endcap 1 and 2 (LE1 and LE2)). The ferromagnetic ring electrode (RE), that is depicted here in black for demonstrative purposes, is also gold-plated in reality. The half-split electrode (UE2) for $Q_{x z}$ excitation is shown. The electrodes are insulated via sapphire rings (grey).

transmission of the mm-waves required to induce a spin flip. The dimensions of the analysis-trap electrodes are given in Table 3.

The analysis trap is a five-electrode trap with only a single set of correction electrodes (see Fig. 20 and Tab. 3). Just like in the case of the precision trap, the analysis trap includes two sets of short endcap electrodes instead of one set of long endcap electrodes to enable adiabatic ion transport. Similarly to the precision trap, the electrodes are made out of OFHC copper and gold plated with the same galvanic process that is mentioned in Section 4.5.2. Owing to the small radius of this trap, and therefore the larger impact of the image charge shift and patch potentials on the surface of the electrodes, the inner surface of the analysis-trap electrodes has been plasma polished to ensure a smoother surface. Unlike the T-shaped insulators that are used in the precision trap for controlled positioning of the electrodes at a temperature of $4 \mathrm{~K}$, here the sapphire insulators keep the same distance between the electrodes, a distance of $140 \mu \mathrm{m}$ whilst the two halves of the split electrode of the trap are kept in distance by spherical quartz glass insulators.

\section{(3) Axial frequency stability}

For unambiguous spin flip determination in the analysis trap, disturbances of the axial frequency need to be minimised since unwanted noise would compromise the stability of the axial frequency. Due to the strong magnetic field inhomogeneity, energy fluctuations of the modified cyclotron mode $E_{+}$would inhibit measurable axial frequency fluctuations. Within this configuration, the influence of the trap voltage is low (due to the low axial frequency) compared to the desirable large influence of the spin state and the influence of $E_{+}$on the axial frequency stability.

The significance of the axial frequency stability in the analysis trap becomes apparent when considering the relative frequency changes (see Tab. 2) that need to be resolved for spin flip detection. In the case of a boronlike argon ion the expected 
Table 3. Electrode dimensions of the analysis trap. This five-electrode trap includes two sets of endcap electrodes instead of one set of double in length endcap electrodes, to enable adiabatic ion transport. For details see text.

\begin{tabular}{lll}
\hline Electrode & Abbreviation & Length $(\mathrm{mm})$ \\
\hline Ring & $R E$ & 0.823 \\
Correction & $L C / U C$ & 2.304 \\
Endcap & $L E_{1,2} / U E_{1,2}$ & 2.996 \\
\hline
\end{tabular}
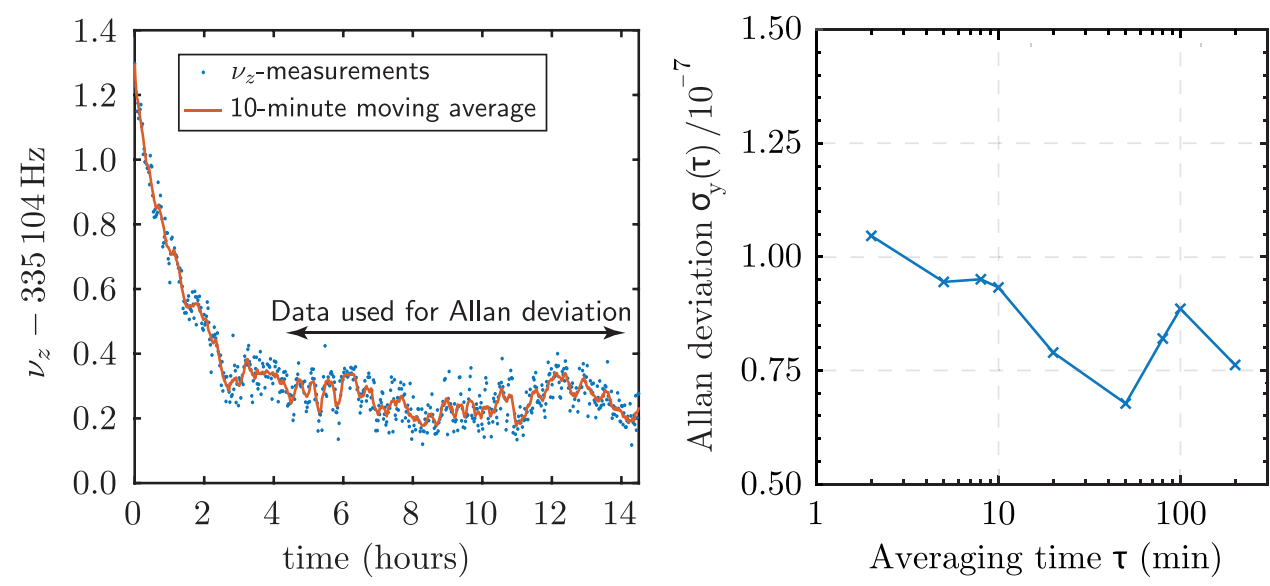

Fig. 21. (Left) Axial frequency stability in the analysis trap over time. Consecutive axial frequency measurements over the course of $14 \mathrm{~h}$ where each data point is obtained every 80 second. The strong drift during the first 3 hours of the measurement shown in the left plot is due to thermalisation of the voltage source, and is plotted for demonstration purposes. (Right) The Allan deviation is plotted with respect to measurement time, excluding the thermalisation data.

frequency jump is $312 \mathrm{mHz}$. To detect a shift of $312 \mathrm{mHz}$ out of the $335 \mathrm{kHz}$ of the ion's axial frequency with high fidelity, we impose the requirement of at least $75 \mathrm{mHz}$ stability. That means that the applied voltage needs to be stable at a $4.5 \times 10^{-7}$ level. This level of stability is provided by a UM1-14 voltage source [76] (for details see Sect. 4.6) and as can be seen in Figure 21 the axial frequency stability requirement is met for ${ }^{40} \mathrm{Ar}^{13+}$, permitting explicit spin flip detection. Measuring the axial frequency consecutively over the course of several hours, the obtained jitter was found to be about $55 \mathrm{mHz}$. In the same figure, the strong drift that occurred during the first $3 \mathrm{~h}$ is due to thermalisation of the voltage source with the environmental conditions of the lab.

\subsection{Trap electronics}

\subsubsection{Axial detectors}

The underlying principle of all measurement schemes performed at ALPHATRAP is the image charge detection technique which was discussed in Section 3.2. The main working horse of this technique is the resonant detection circuit. It consists of a parallel 
RLC tank circuit (resonator) with resonance frequency $\omega_{\text {res }} \approx \frac{1}{\sqrt{L C}}$ and impedance

$$
Z(\omega)=\left(\frac{1}{R_{\mathrm{p}}}+i\left(\frac{\omega}{L \omega_{\mathrm{res}}^{2}}-\frac{C \omega_{\mathrm{res}}^{2}}{\omega}\right)\right)^{-1}
$$

followed by an ultra-low-noise cryogenic amplifier. Here $R_{\mathrm{p}}=Z\left(\omega_{\text {res }}\right)$ denotes the equivalent parallel resistance which is a figure of merit for a high signal-to-noise ratio and thus an efficient and fast ion detection. $R_{\mathrm{p}}$ can be directly determined from the quality factor

$$
Q=\frac{\omega_{r e s}}{\Delta \omega}=\frac{R_{\mathrm{p}}}{\omega_{r e s} L}
$$

where $\Delta \omega$ is the $-3 \mathrm{~dB}$ bandwidth of the resonator. Electrically $R_{\mathrm{p}}$ represents the combined component losses of the detection circuit and can be expressed in terms of the capacitor's equivalent series resistance (ESR) and the inductor's equivalent winding resistance $R_{L}$ at the respective frequency:

$$
R_{\mathrm{p}} \approx \frac{L}{C\left(\mathrm{ESR}+R_{L}\right)} .
$$

According to this relation, $R_{\mathrm{p}}$ can be increased by minimizing the circuit parameters $C, R_{L}$ and ESR and maximizing $L$. Typically, these quantities are not independent and are also partly restricted by the boundary conditions of the experiment. In order to reach maximal detection efficiency all detection circuits are therefore highly optimised in terms of their geometry and material selection resulting in unloaded $Q$-values of up to 324000 [77], which corresponds to an equivalent parallel resistance of $R_{\mathrm{p}} \approx 6.5 \mathrm{G} \Omega$.

At Alphatrap, the analysis trap and precision trap are both equipped with their own purpose built axial detector circuit. The resonators are realised as helical inductors wound on cylindrical PTFE coil formers and screened by cylindrical housings from external influences. Shield and wire are made from the type-II superconductor $\mathrm{NbTi}$, allowing for minimal conductive losses [77] when operated below its critical temperature. The resonators are positioned in a magnetic field of $B \approx 2 \mathrm{~T}$ which corresponds to a critical temperature of $T_{\mathrm{c}} \approx 8 \mathrm{~K}$.

The basic construction of the AlPhATRAP resonators is shown in Figure 22. The circuit parameters of all resonators are summarised in Table 4 . The analysis-trap and precision-trap resonator have an identical housing geometry and only differ by the coil dimension and the number of windings. These are chosen such that in combination with parasitic wire capacitance and the respective trap capacitance the final resonance frequency of the precision-trap detection circuit is $651.4 \mathrm{kHz}$. It allows for detection of ions with $q / m>1 / 4 \mathrm{e} / \mathrm{u}$, limited by the currently maximal accessible trapping voltage of $-100 \mathrm{~V}$.

For the non-coherent dip detection, which is used during the axial frequency measurement in the precision trap, a high signal-to-noise ratio and therefore a high $R_{\mathrm{p}}$ is of great importance since it significantly reduces the necessary measurement time. On the other hand the statistical precision is limited by the line width of the dip signal $\delta \nu=\frac{1}{2 \pi \tau}$ which scales linearly with $R_{\mathrm{p}} \cdot q^{2}$ (see Eq. (16)). To counteract this limitation the precision-trap detector is equipped with a dedicated $Q$-switch (see Fig. 22). In high- $Q$ mode the complete precision-trap detector features a signal-tonoise ratio of $24 \mathrm{~dB}$ at a final $Q$-value of $40000\left(R_{\mathrm{p}} \sim 344 \mathrm{M} \Omega\right)$. In combination with an effective electrode distance of $29.3 \mathrm{~mm}$ this translates into a dip width of $\sim 4.1 \mathrm{~Hz}$ for a single ${ }^{40} \mathrm{Ar}^{13+}$ ion. 

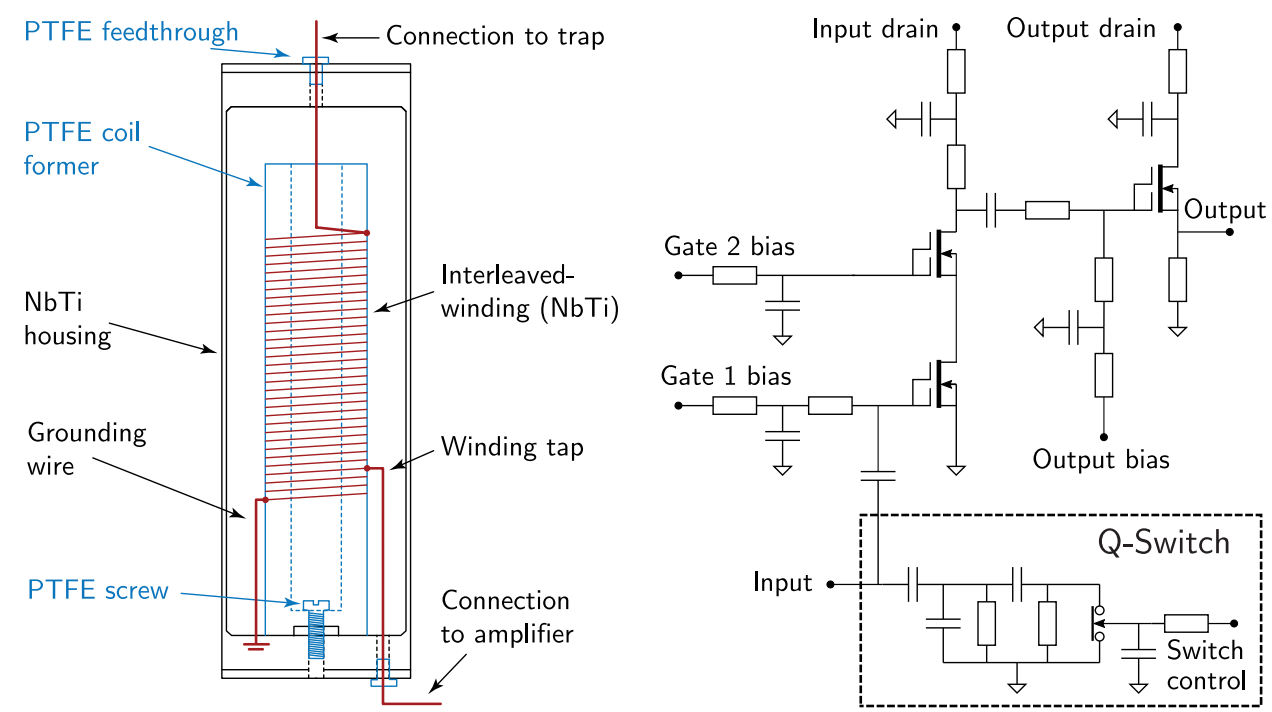

Fig. 22. (Left) All Alphatrap resonators have a similar housing geometry. They are $12.8 \mathrm{~cm}$ long and $4 \mathrm{~cm}$ in outer diameter, limited by spatial constraints of the experiment. The NbTi wire is arranged in a so-called interleaved winding which features a small parasitic winding capacitance. All insulating parts including coil former and wire insulation are made from PTFE due to its small permittivity $\left(\epsilon_{r}=2.1 \mathrm{Fm}^{-1}\right)$ and low loss angle $\left(\tan \delta<10^{-4}\right)$. (Right) Layout of the precision-trap axial amplifier and $Q$-switch. In low- $Q$ mode the $Q$ switch is open and the effective parallel resistance is limited by the parallel impedance of the snubber (damping) circuit. In high- $Q$ mode the switch is closed and shorts the damping resistor to ground.

Table 4. The circuit parameters of all Alphatrap resonators, where $N$ denotes the winding number, $L$ the effective inductance, $C$ the effective capacitance. $Q$ loaded and $R_{p}$ loaded denote the final cryogenic values of the complete detector circuits (connected to the trap and the amplifiers) inside the magnetic field.

\begin{tabular}{llll}
\hline & Precision-trap axial & Analysis-trap axial & Precision-trap cyclotron \\
\hline$N$ & 684 & 1912 & 10 \\
$L$ & $2.1 \mathrm{mH}$ & $10.5 \mathrm{mH}$ & $1.3 \mu \mathrm{H}$ \\
$C$ & $5.1 \mathrm{pF}$ & $6.3 \mathrm{pF}$ & $4 \mathrm{pF}$ \\
$Q$ loaded & 40000 & 7000 & 1800 \\
$R_{\mathrm{p}}$ loaded & $344 \mathrm{M} \Omega$ & $155 \mathrm{M} \Omega$ & $0.37 \mathrm{M} \Omega$ \\
\hline
\end{tabular}

Figure 23 shows the effect of the $Q$-switch on the noise resonance of the precisiontrap detector and the dip width of a single ${ }^{40} \mathrm{Ar}^{13+}$ ion. The $Q$-switch is also switched to low- $Q$ mode during particle production and sideband cooling. It also increases the cooling time constant and enables an extension of the evolution time during phase resolved detection.

Compared to the precision-trap the analysis-trap detection circuit is designed for a lower detection frequency of approximately $335.1 \mathrm{kHz}$. This can be attributed to the fact that the spin flip induced axial frequency jump in the analysis trap scales with $1 / \nu_{z}$. However, if the detection frequency would be chosen too small this could impair the trap harmonicity due to an increased influence of patch potentials compared to the relatively small trapping voltage. The chosen detector frequency is also the lower frequency limit without sacrificing the signal-to-noise ratio due to the amplifiers $1 / f$ noise. 


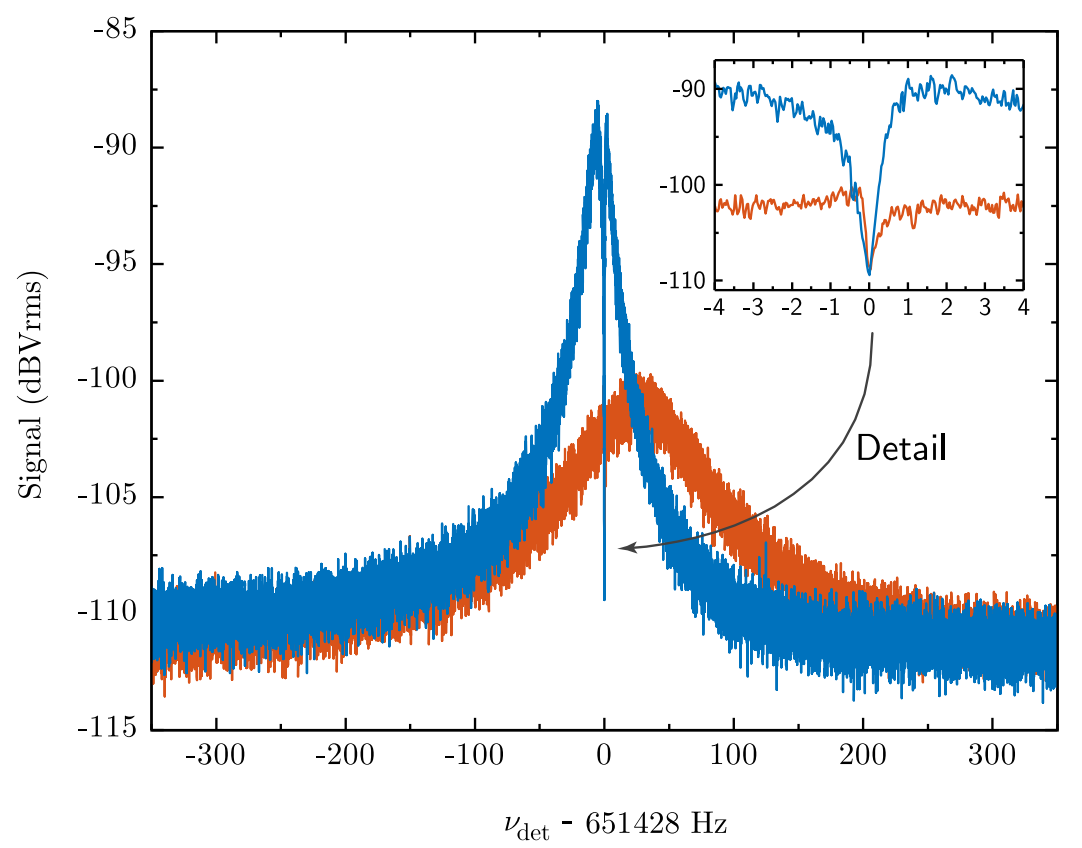

Fig. 23. Noise resonance of the precision-trap detection circuit in high $Q$ (blue) and low $Q$ (orange) mode. In low $Q$ mode $R_{\mathrm{p}}$ and therefore also the width of the visible ${ }^{40} \mathrm{Ar}^{13+}$ ion dip is reduced by a factor of $\sim 5$. The parasitic capacitance of the active $Q$-switch shifts the detector's resonance frequency by about $31 \mathrm{~Hz}$. The inset is a zoom of the dip position.

Each axial resonator is connected to its own cryogenic amplifier which are identical in design (see Fig. 22). They are based on GaAs MESFETs which feature a high-input impedance and low-noise characteristics at liquid helium temperatures. Care is taken in order to keep the amplifier's parasitic influence on the resonator as small as possible. The input stage is therefore designed in a discrete common-source cascode configuration. It significantly reduces the amplifier's back-action on the resonator's quality factor caused by the parasitic Miller capacitance. A source follower in the output stage provides impedance matching to the coaxial lines which are used for signal transport. Compared to the unloaded resonator the axial amplifiers have a relatively small effective input resistance of about $22 \mathrm{M} \Omega$ at their operation frequencies. It would constitute a major limitation if the amplifier input was directly connected to the resonator which would correspond to a coupling factor of $\kappa=1$. For this reason the AlPhATRAP axial amplifiers is decoupled from the resonator by tapping the resonator coil according to an autotransformer with winding ratio $N_{\mathrm{S}} / N_{\mathrm{P}} \approx 1 / 10$. Here $N_{\mathrm{S}}$ denotes the secondary winding number and $N_{\mathrm{P}}$ the primary or total winding number.

\subsubsection{Cyclotron detector}

In principle, by utilizing sideband coupling, one axial detector per trap is sufficient for the determination of all eigenfrequencies. However, a dedicated cyclotron detector is useful for the direct detection and an efficient bolometric cooling of the modified cyclotron motion. Therefore, the precision trap is also equipped with a cyclotron detector circuit. 
Compared to the axial detectors the cyclotron detector circuit is optimised for much higher operation frequencies in the order of $20 \mathrm{MHz}$ to $30 \mathrm{MHz}$ depending on the ion's charge-to-mass ratio (Eq. (5)). Just as the axial resonators the cyclotron resonator also features a helical coil geometry and is built into a similar NbTi housing. The coil is made from multistranded NbTi wire. Using multiple thin insulated wires instead of a single thick wire reduces conduction losses caused by the skin effect. Since at $300 \mathrm{~K} \mathrm{NbTi}$ has a considerable specific resistance of $\rho_{s} \approx 0.7 \mu \Omega \mathrm{cm}$ [78], this measure ensures a normal conducting quality factor of $Q \approx 80$ and allows for easier frequency adjustment during maintenance at room temperature. The cyclotron amplifier is based on a design discussed in [79].

For later in situ frequency tuning the cyclotron detector is equipped with a capacitively decoupled varactor diode. It allows for a compensation of typical frequency shifts on the order of $\sim 0.5 \mathrm{MHz}$ caused by thermal contraction processes during the cooldown and thermalisation of the experiment. It also enables an adjustment of the cooling time constant during phase resolved detection of the modified cyclotron frequency. During a precision measurement, the varactor allows us to detune the detector from the ion, this way reducing the adverse effect of frequency pulling, which shifts the ion's cyclotron frequency as a result of the interaction with the cyclotron detector.

\subsubsection{Charge amplifiers}

Efficient dynamic capturing of externally produced HCI in the capture section (see Sect. 4.5.1) requires precise information on the energy and arrival time of the ion bunch in the order of microseconds. For this reason Alphatrap is equipped with two cryogenic charge sensitive detectors. They include two charge amplifiers each connected to a Faraday cup (FC) situated at the upper and lower end of the trap tower. Figure 24 shows the post-amplified averaged signal of the two charge sensitive detectors during the injection of $\mathrm{Ar}^{9+}$ bunches. The dispersive signal picked up by FC1 indicates that the ion bunch is sufficiently compressed in radial direction in order to pass the $3 \mathrm{~mm}$ hole of the diaphragm without significant ion loss.

The signal on the second detector shows an $\mathrm{Ar}^{9+}$ bunch impinging on the target of the in-trap EBIS which was used as the second FC at that time. Besides an additional discharge resistor at the input the charge amplifiers do not differ from the axial amplifier layout. At a gain of $14 \mathrm{~dB}$ and a Faraday cup capacitance of about $12 \mathrm{pF}$ the output charge sensitivity of each detector amounts $418 \mathrm{mV} / \mathrm{pC}$ or $67 \mathrm{nV}$ per elementary charge.

\subsubsection{DC-biasing}

For the DC-biasing of the trap electrodes and cryogenic electronics ALPHATRAP is equipped with four different types of voltage sources. The ring and correction electrodes of the precision trap are individually supplied with up to $-100 \mathrm{~V}$ from five ultra-stable channels of a StaReP (Stable Reference for Penning-trap experiments) voltage source developed in house [80]. The voltage resolution of each channel is defined by two 16bit DACs (digital-to-analog converter), one for the coarse and one for the fine-voltage setting. The relative voltage stability is in the order of $10^{-8}$ for a typical time scale of 10 minutes.

The analysis-trap ring and correction electrodes are connected to three 25-bit precision channels of a UM1-14 voltage source from Stahl Electronics, which shows relative fluctuation on a $10^{-7}$ level. Additionally the UM1-14 includes ten 16-bit fast mode channels that are used for the analysis-trap endcaps and for controlling the solid state excitation line switches. The remaining transport electrodes as well as 


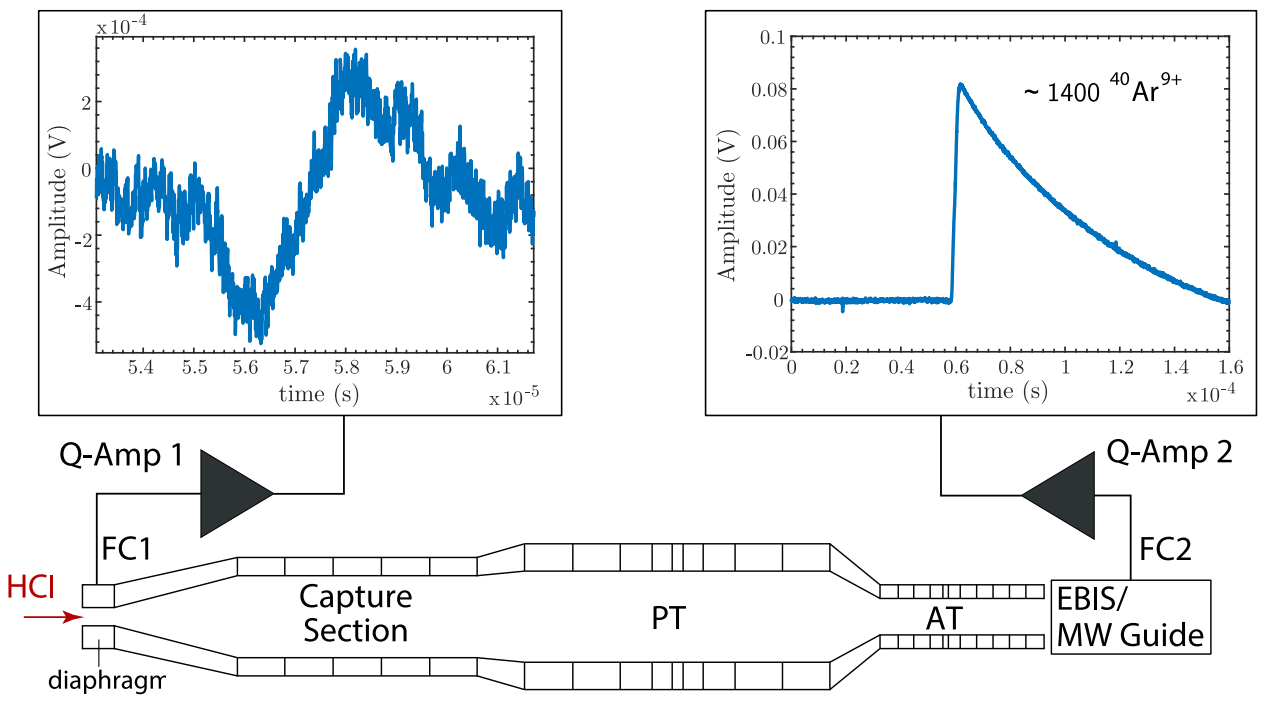

Fig. 24. Typical averaged signals of slow $(100 \mathrm{eV}) \mathrm{Ar}^{9+}$ bunches visible on the two charge sensitive detectors installed at the entrance and exit of the trap tower. The signal amplitude corresponds to $\geq 1400 \mathrm{Ar}^{9+}$ ions. The bunches arrive at $\mathrm{FC} 2$ about $0.6 \mathrm{~ms}$ after the ejection from the HC-EBIT. The signal rise time corresponds to an ejection pulse time (temporal bunch length) of $\sim 3 \mu \mathrm{s}$.

the cryogenic amplifiers are supplied by two bipolar LoCepps (Low Current enabled precision power supply) sources which have been developed in our group and are described in [81]. The high positive blocking voltages that are required in the capture section during the injection of ions are delivered by a bipolar HV 200-8 supply from Stahl Electronics.

On their way to the trap electrodes and the cryogenic electronics, the DC lines run through three RC lowpass filter stages located at $300 \mathrm{~K}, 77 \mathrm{~K}$ and $4.2 \mathrm{~K}$ (compare Fig. 25). The serial connection of the three successive filter stages corresponds to an effective cutoff frequency of $28 \mathrm{~Hz}$ and an effective filter time constant of $6.8 \mathrm{~ms}$. The high voltages needed in the capture section are filtered by dedicated HV filters on a separate HV board. The filters of the switchable capture electrodes are bypassed with antiparallel diode pairs. This way, a fast switching $(\sim 1 \mu \mathrm{s})$ of voltages exceeding the diode threshold voltage becomes possible, while typical noise amplitudes are still efficiently filtered by the RC filter.

\subsubsection{AC-excitations}

Figure 25 shows a simplified circuit diagram of the precision trap. For the excitation and coupling of the individual particle eigenmodes, the Alphatrap experiment is equipped with 5 excitation lines, including two quadrupolar and two dipolar excitations. Additionally, electronic feedback can be applied through a shared line which is capacitively coupled to the inputs of all detector circuits.

The excitations as well as the amplifier output signals are transported via cryogenic coaxial cables made from stainless steel. Despite the relatively low thermal conductivity of stainless steel, due to the rather large cable dimensions, the additional thermal load introduced by the coaxial lines amounts to about $20 \%$ of the total heatload on the LHe cryostat. All excitation lines are connected to the trap 


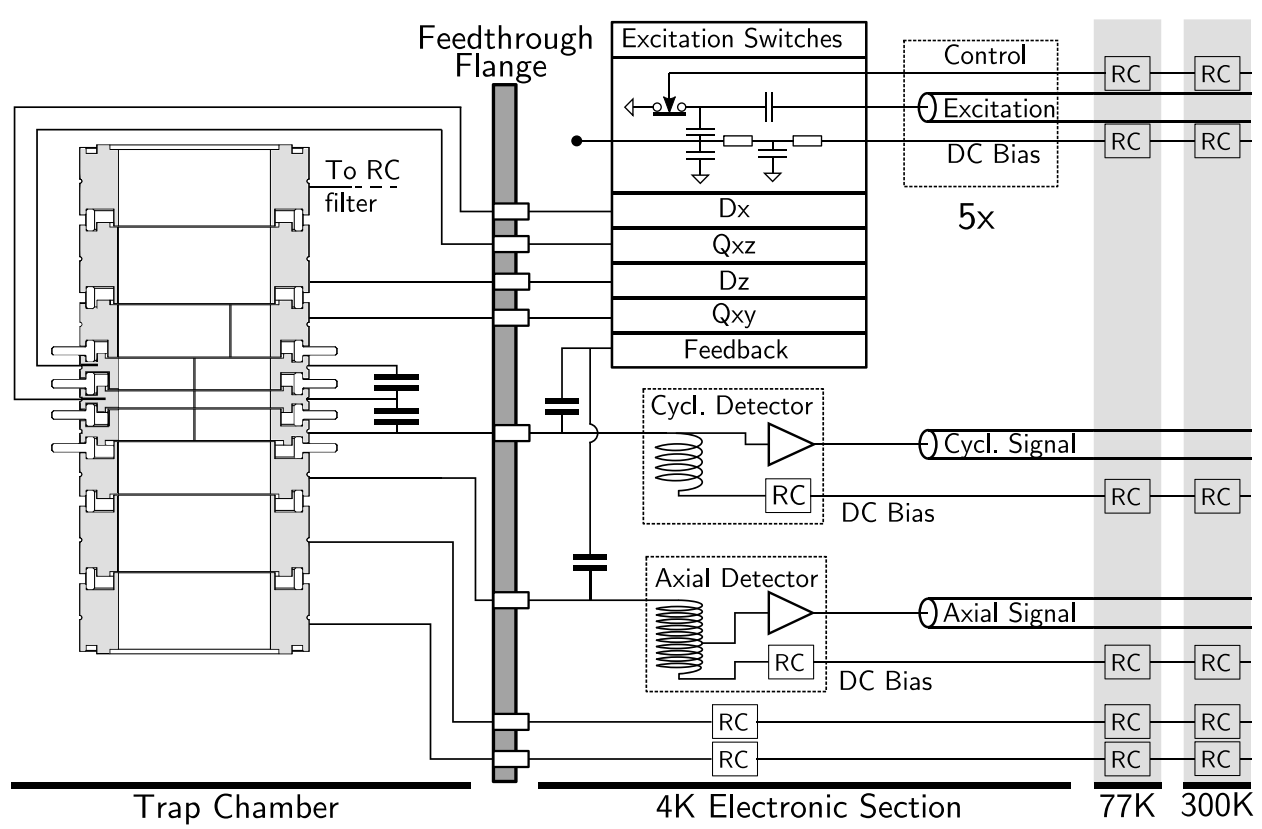

Fig. 25. Simplified wiring diagram of the precision trap. The detector circuits as well as the excitation switches include the DC biasing of the respective electrodes. RF block resistors protect the excitation signal from AC shorts to ground. To increase the cyclotron signal the split ring and correction electrodes are capacitively interconnected within the trap chamber. For reasons of clarity, not all bias connection are shown here.

electrodes via cryogenic solid-state switches. In "on" state, i.e. during the application of an excitation the switches are high ohmic and the signal can pass. However, while the excitation lines are not in use the switches act as AC short to ground. This allows reducing parasitic radiofrequency noise which enters through the excitation lines. At $25 \mathrm{MHz}$ the signal and therefore also the noise attenuation difference between on and off state amounts to more than $40 \mathrm{~dB}$.

\subsection{The millimeter-wave setup and laser access}

As mentioned in Section 3.6 the spin flip spectroscopy method employed in ALPHATRAP requires the possibility to irradiate the ion with a suitable millimeterwave radiation. In the magnetic field of the superconducting magnet of about $4 \mathrm{~T}$ the Zeeman splitting for the $s$-ground state of hydrogenlike ions amounts to about $112 \mathrm{GHz}$, however for a $p_{1 / 2}$-ground state as e.g. in boronlike ion systems it only amounts to one third of this value to about $37.3 \mathrm{GHz}$. These frequencies are generated by a microwave synthesizer (Anritsu MG3694C) directly up to $40 \mathrm{GHz}$. For frequencies corresponding to electronic $s$-states, the fundamental frequency output of the synthesizer is multiplied by an active multiplier chain (OML S10MS). The synthesizer is referenced to a $10 \mathrm{MHz}$ signal from a rubidium frequency standard (Stanford Research Systems, FS725).

In order to couple these microwaves into the Penning-trap tower, waveguides are used (see Fig. 26). In the design for the waveguides care was taken to allow operation for both frequency bands with a minimum of change necessary when changing frequency band [82]. Since waveguides for $37 \mathrm{GHz}$ would not allow for a single mode operation at $112 \mathrm{GHz}$, a tapered transition at the end of the waveguide acts as a mode 


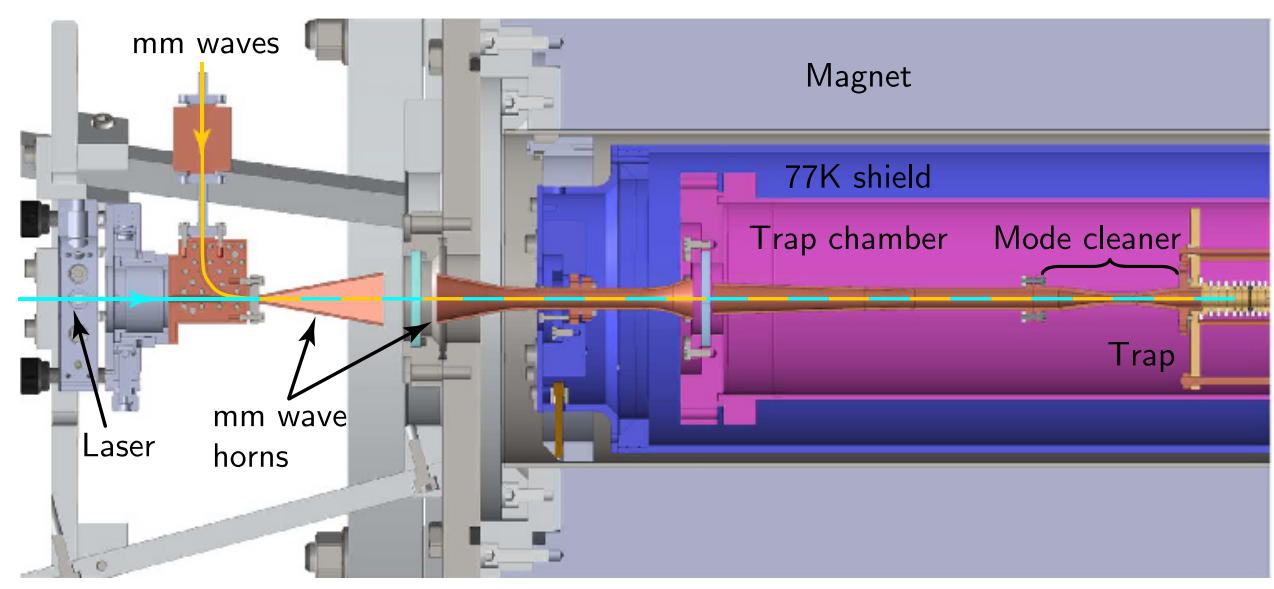

Fig. 26. Cross sectional view of the microwave injection system. The different regions of the temperature shields are color coded (blue: liquid nitrogen at $77 \mathrm{~K}$, pink: liquid helium at $\sim 4 \mathrm{~K})$. In cyan the path of the incoupled laser light is shown. The figure has been rotated by 90 degrees.

cleaner, which has to be exchanged with a straight section when switching between the two frequency bands.

The microwaves are coupled into the trap from below the magnet vertically upwards into the magnet bore. On the way the microwaves propagate through three different pressure regions, from atmospheric pressure into the insulation vacuum of the cryostat into the cryogenically pumped extremely high vacuum of the trap chamber. This can be accomplished by horn to horn transitions, as shown in Figure 26. The horns are thermally anchored at the $77 \mathrm{~K}$ shield and at the trap chamber at $4 \mathrm{~K}$, respectively. Between two horns a wedged window made of UV fused silica is placed, which has very good transmission for both the microwaves as well as light over a broad spectral range from visual down to near ultraviolet. These windows allow the possibility to simultaneously insert also a laser beam which can then be used for e.g. Doppler laser cooling as described in Section 3.8 or laser spectroscopy of HCI, see Section 6.3. The laser light for Doppler laser cooling and spectroscopy are produced by different laser sources in a dedicated laser laboratory adjacent to the magnet room. From there the beam can be transported to the area below the superconducing magnet via a free beam or in a fiber. It arrives on an optical breadboard which can house different optics for beam preparation and steering for alignment purposes. To this end, the millimeter waves are bent by $90^{\circ}$, while the laser beam propagates straight. Whereas for the lower frequency respectively long wavelength a small pinhole for the laser light can be drilled into a waveguide bend without compromising the transmission, this is not directly applicable for shorter wavelengths. Therefore a self-designed waveguide bend is used, which allows for an acceptable microwave transmission and at the same time features a moderate sized aperture for the laser beam. Simulations done with the RF Module of COMSOL Multiphysics predict a microwave power transmission in the desired frequency around $112 \mathrm{GHz}$ of more than $75 \%$, which would allow to couple in enough microwave power for inducing a spin flip on time scales of few milliseconds.

\subsection{The cooling laser system}

For sympathetic laser cooling of the HCI under investigation, at least one further ion with a suitable electronic structure is necessary. Only very few elements exhibit 
in their ionic states a suitable transition in the range accessible by lasers. A small charge-to-mass ratio $q / m$, similar to the value for highly charged ions (e.g. $q / m \approx$ $0.39 \mathrm{e} / \mathrm{u}$ for ${ }^{208} \mathrm{~Pb}^{81+}$ ), is desirable to keep differences in trap frequencies as small as possible. The single ionised charge state of ${ }^{9} \mathrm{Be}$ has a charge-to-mass ratio of $q / m \approx$ $0.11 \mathrm{e} / \mathrm{u} .{ }^{9} \mathrm{Be}^{+}$exhibits a suitable electronic transition between the ${ }^{2} \mathrm{~S}_{1 / 2}$ and ${ }^{2} \mathrm{P}_{3 / 2}$ states with a zero-field wavelength of $313.132922(44) \mathrm{nm}$ [56]. The lifetime of the excited state is 8.1(4) ns [50] resulting in a natural line width of the excited state of $\Delta \nu_{\text {nat }}=19.7(10) \mathrm{MHz}$. The saturation intensity of this transition is $836(42) \mathrm{W} / \mathrm{m}^{2}$. To illuminate the trap at this intensity with a beam of $0.95 \mathrm{~mm}$ diameter, corresponding to the diameter of the smallest used millimeter waveguide, less than $0.6 \mathrm{~mW}$ are necessary.

A precise frequency control of the laser system is required for laser cooling. This is achieved by locking the laser to a frequency reference. Contrary to most experiments using the fluorescence light emitted by the laser-cooled ion for wavelength locking, the requirements on the electric field homogeneity and the possible coverage of solid angle do not allow the use of photodiodes in the current ALPHATRAP trap tower. An external frequency measurement with sufficient accuracy connected to a regulation loop will be used instead. It is characterised by a frequency accuracy of $2 \mathrm{MHz}$ and a sample time of the stabilization control loop of $100 \mathrm{~ms}$. Fluctuations occurring on timescales below the stabilization sampling time contribute to the line width measured with an corresponding integration time. To ensure reliable Doppler cooling and avoid heating through frequency fluctuations into the blue detuned side of the electronic transition, the laser system's line width should be significantly smaller than $\Delta \nu_{\text {nat }}$.

The system consists of a commercial TA-FHG pro system manufactured by Toptica Photonics, an accurate HighFinesse Ångstrom WS Ultimate 2 wavelength meter and an LJSC-3-11 frequency reference laser developed by Lasertex [83-85]. This laser system uses a diode laser with small linewidth and fourth harmonic generation to produce the light for the cooling of the ${ }^{9} \mathrm{Be}^{+}$ions. Via viewports in the room temperature and cryogenic section of the cryostat the light can be injected into the trap tower, see Figure 26. An External Cavity Diode Laser (ECDL) in Littrow configuration emits the fundamental light at $1252 \mathrm{~nm}$. The extended length of the resonator as well as the small bandwidth of the grating allow single-mode operation with a narrow line width compared to other common types of laser systems [86]. It has been measured with a $3 \mu$ s integration resulting in a line width below $7 \mathrm{kHz}$. A piezo allows adjusting the length of the cavity in order to stabilise the wavelength. A Tapered Amplifier (TA) active medium amplifies the typical output power of $85 \mathrm{~mW}$ of the fundamental light to $1.8 \mathrm{~W}$, retaining the spectral characteristics of the ECDL. Frequency conversion takes consecutively place in two cavities equipped with birefringent crystals. The stabilization of the length of the cavity uses a Pound-Drever-Hall lock. The second harmonic generating cavity (SHG) delivers $800 \mathrm{~mW}$ of light at a wavelength of $626 \mathrm{~nm}$ while $300 \mathrm{~mW}$ are available after fourth harmonic generation (FHG). Figure 27 shows a schematic drawing of the complete laser system. This allows easily to reach and surpass the saturation intensity and can compensate for large beam transport losses. At integration time scale of $100 \mathrm{~ms}$ under laboratory conditions, the laser light at $313 \mathrm{~nm}$ exhibits a linewidth of $191(3) \mathrm{kHz}$ [57]. On larger timescales, the wavelength is actively stabilised to the desired value. The output of a wavelength meter using a set of Fizeau-type interferometers is used as process variable for a Proportional-Integral-Derivative controller acting on the piezo-adjustment of the ECDL cavity. The wavelength meter is characterised by a measurement resolution of $500 \mathrm{kHz}$ and an absolute accuracy of $2 \mathrm{MHz}$ inside $10 \mathrm{~nm}$ around calibration point. In order to assure this accuracy, every $2 \mathrm{~min}$ recalibration of the wavelength meter with a stable reference is necessary [84]. The Helium-Neon-laser system serves as calibration light source. Intra-cavity spectroscopy locks the laser system to one 


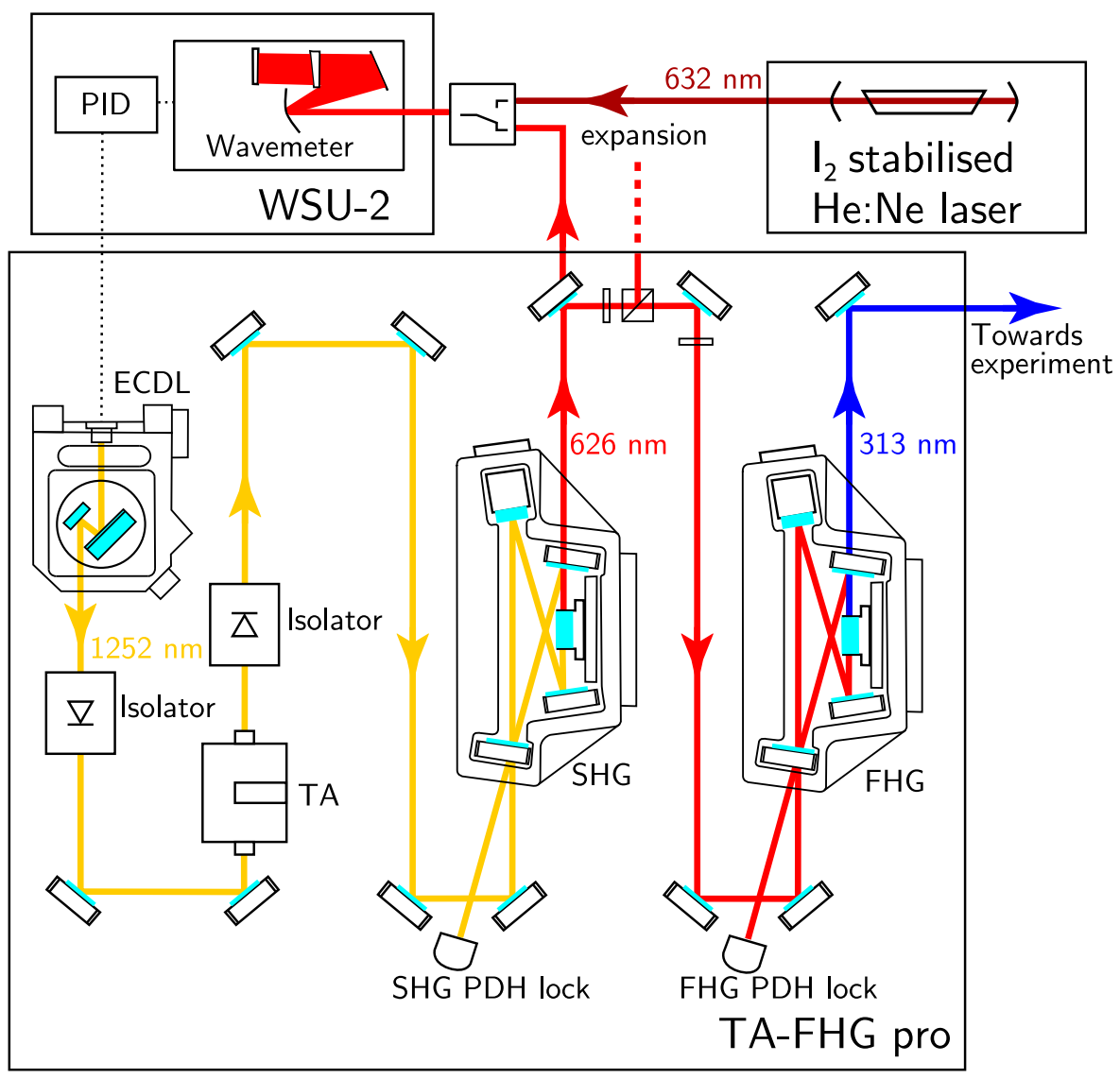

Fig. 27. Overview over the setup of the cooling laser system. Fundamental light from an external cavity diode laser (ECDL) is amplified in a tapered amplifier (TA) and frequency doubled in the second harmonic generation cavity (SHG) and fourth harmonic generation cavity (FHG). A wavelength meter calibrated with a Helium-Neon laser reference feeds a PID regulation loop that controls the ECDL. Adapted from [87].

of 14 hyperfine transitions of molecular iodine in the vicinity of $632.991 \mathrm{~nm}$. The absolute frequency uncertainty amounts to typically $12 \mathrm{kHz}$ and the frequency stability is specified to be $\Delta \nu / \nu=1 \times 10^{-11}$ for 1 s averaging time [85]. This means the calibration contributes a very small uncertainty compared to the wavemeter itself.

Frequency stabilization to below $2 \mathrm{MHz}$ on timescales larger than $100 \mathrm{~ms}$ combined with a small intrinsic line width on lower timescales is expected to allow laser cooling without fluorescent feedback from the ion stored in the precision trap.

\subsection{Control system}

The ion-trap control system is based on the scripting language and development environment Matlab. Matlab had previously been in use at the experiment for data analysis. Experience with previous ion-trap systems has shown that scripting capability is highly advantageous for running automated measurements, so a scripting language was chosen for the control system. Using the same language and development environment for trap control and data analysis reduces the training time for new 
students and enables tight interaction between data analysis and data taking. Matlab scripts do not need to be compiled and can be modified at run-time, which enables fast iteration, and the text-based nature of the files is advantageous for working with version-control software.

In order for the code to be self-documenting, the experiment hardware is described in a single "trap_define.m" script, which assigns names to all function generators, voltages sources, electrodes, detectors, and electrodes, and defines the relationships between them. Each physical device is represented as an object derived from the "handle class" to ensure the state of the software object always corresponds to the state of the hardware. ${ }^{3}$ All this information is collected into a single object called "trap", which is passed to all helper functions. Measurements are performed by writing scripts that calls the appropriate helper functions. These measurement scripts and the "trap_define.m" script are committed into a version-control system, which helps to document the evolution of the experiment alongside the current measurements.

Instrument communication is based on the VISA-library for most commercial devices (requires the "Instrument Control Toolbox"), and on serial communication and TCP sockets for custom-built equipment. This way, the control system structure is kept generic to allow porting the system to a different scripting language, such as Python, if the need arises.

\section{Commissioning of the trap system}

\subsection{Cleaning and preparation of a single ion}

After ion production, several species in different abundances are potentially present in the trap. In Figure 28 a typical mass scan can be seen. This particular ion cloud has been taken after operating the mini Electron Beam Ion Source (see Sect. 4.4.3) for intrap ion production. After 15 seconds of breeding time the electron gun is turned off. The following ring voltage scan allows ion identification via the different frequencies of the axial oscillation for the different charge-to-mass ratios present in the trap. Hot ions generate a large signal when the axial frequency passes by the resonator, which is plotted versus the ring voltage in Figure 28.

Having an ion bunch in the precision trap which was either internally produced using the in-trap EBIS (see Sect. 4.4.3) or externally produced, injected and captured (see Sect. 4.5.1), different techniques exist for reducing the ion cloud population down to a single ion. Axial cleaning was implemented where strong rf-excitation sweeps are applied on all frequencies except the axial frequency of the ion of interest. That way, unwanted species are excited to large amplitudes before the applied confining potential is shortly relaxed, so that the energetic ions are no longer axially confined. This technique is effective towards impurities with $q / m$ different to the ion of interest, in this case ${ }^{12} \mathrm{C}^{5+}$, as shown in Figure 29.

Another possibility to reduce the ion population down to the one of interest, is by addressing the magnetron mode. With this technique, a wideband excitation in the form of noise close to the magnetron frequency is applied. Only the magnetron motion of the ion of interest is simultaneously cooled using the axial-magnetron sideband, in order to cancel the effect of the applied noise. This way all unwanted species are eventually lost radially to the walls of the trap, leaving the ion(s) of interest confined inside the trap.

Since the width of the noise dip scales linearly with the number of ions resonant with the detection system (see exemplary measurement in Fig. 30), we can extract

\footnotetext{
${ }^{3}$ In Matlab, all objects are passed-by value, except for objects derived from a fundamental pointerlike object, the "handle" class.
} 


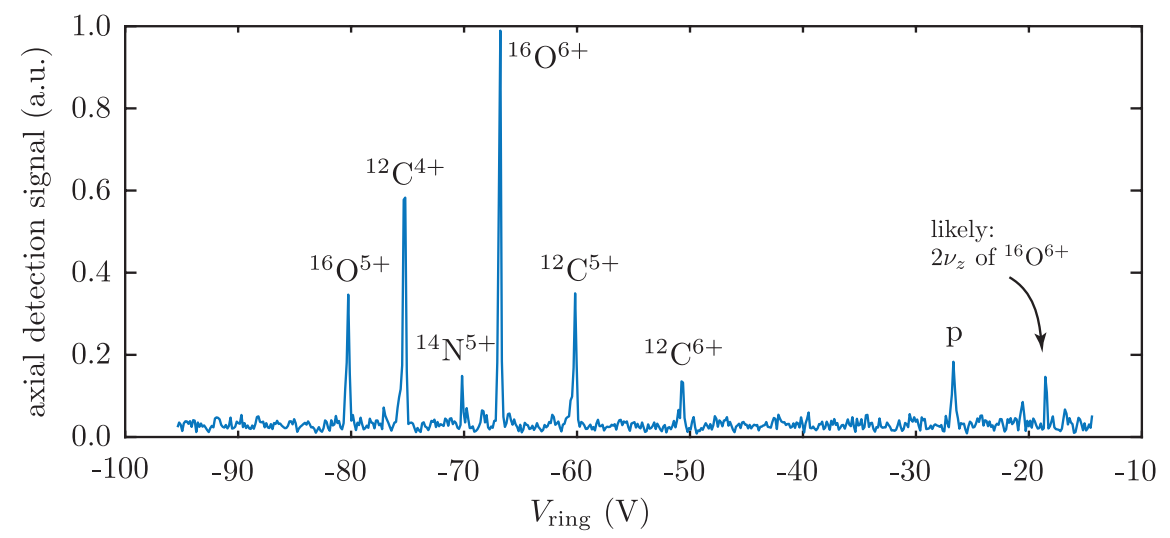

Fig. 28. Example mass spectrum after in situ ion production in the precision trap using the in-trap EBIS. Predominantly, ions of carbon, the target material, and oxygen in different charge states as contaminant can be identified.

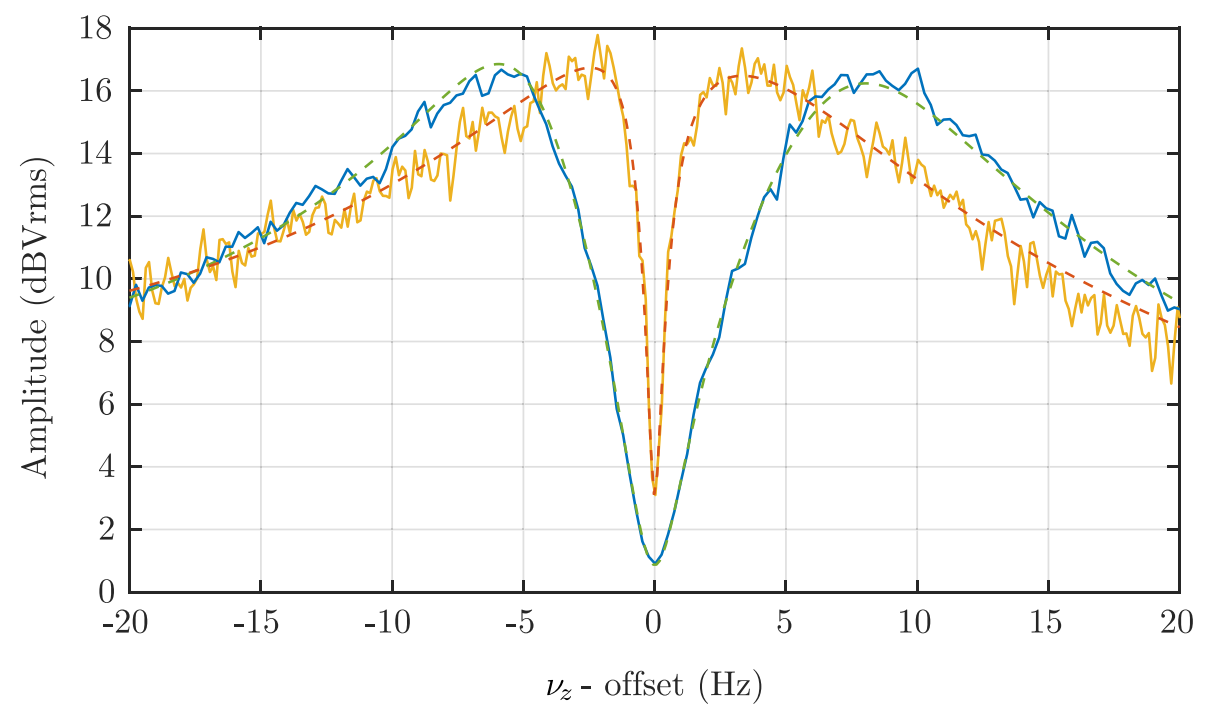

Fig. 29. A single ${ }^{12} \mathrm{C}^{5+}$ ion on resonance with the detection system (yellow line). The fit of this signal is also shown with the dashed red line. Five ions of the same species on the resonator (blue line) and the signal's fit (green dashed line).

the number of ions inside the trap from a fit to the dip. When the remainder trapped ion cloud consists only of ions of the same charge-to-mass ratio, then by applying a cyclotron burst excitation on the modified cyclotron frequency of the ion species, all existing ions are excited to slightly different amplitudes. Consequently, each ion appears as an individual peak on the cyclotron resonator (see Sect. 4.6). In this configuration it is possible to address individual ions by individual chirped cyclotron excitations. Finally, the hot ions can be removed from the trap either by exciting the now individually shifted axial frequencies or by simply lowering the trap potential and waiting for collisions of the hot ions. A good signature for the success of the cleaning procedure is shown in Figure 29, where the dip width of the signal shown in the yellow spectrum corresponds to a single ion. Especially in the analysis trap, the stability of the axial frequency is a good indicator for remaining contaminants as any 


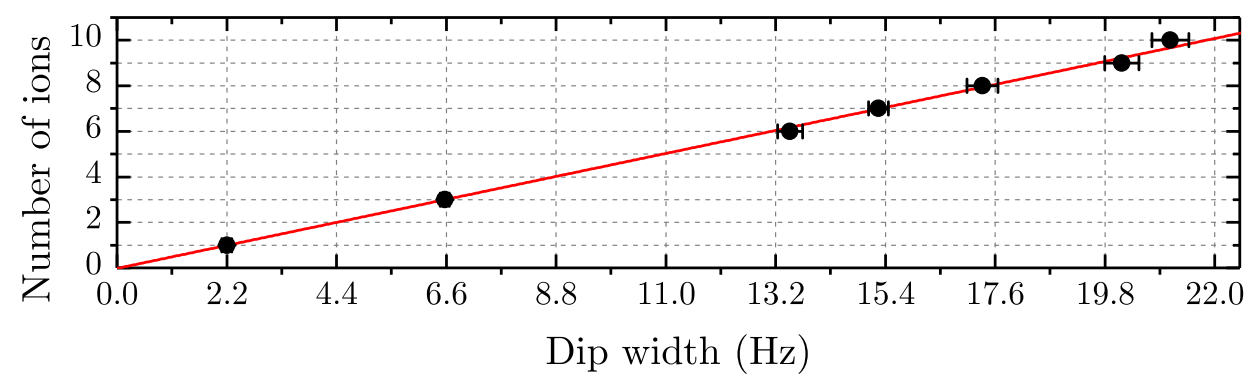

Fig. 30. The number of trapped ${ }^{12} \mathrm{C}^{5+}$ ions in the precision trap versus the width of the detected dip. The width of the dip generated by the common motion of the ions is always an integer multiple of the width of a single ion, about $2.2 \mathrm{~Hz}$ in the case of ${ }^{12} \mathrm{C}^{5+}$.

interactions between different ions would inevitably lead to frequency fluctuations in the strong magnetic bottle.

\subsection{Electric field optimisation}

After the preparation of a single ion, the trap's electrostatic potential has to be optimised. Due to the finite machining precision and unavoidable variations of the potential on the electrode surfaces, the potential will differ slightly from the calculated one. This optimisation is essential for all following measurements.

As mentioned in Section 3.1 the $C_{n}$ coefficients (Eq. (8)) that characterise the leading order unharmonicities of the electric field $\left(C_{4}, C_{6}, C_{8}\right.$ and $C_{10}$ in the case of Alphatrap ) can be minimised by applying suitable correction voltages. To do that, the ion's axial frequency was monitored while the radius of the ion's magnetron motion was altered. In the presence of electric field imperfections, the axial frequency shift by the dominant $C_{4}$ contribution is given by [88]:

$$
\frac{\Delta \nu_{z}}{\nu_{z}}=\frac{C_{4}}{C_{2}} \frac{3}{4 d^{2}}\left(z^{2}-2 \rho_{+}^{2}-2 \rho_{-}^{2}\right)
$$

in first order. Since the precision trap is a 7-electrode trap, which requires a set of two independent correction voltages, it needs to be dynamically compensated. In other words, the trap is not orthogonal ${ }^{4}$ with respect to the individual correction voltages. When adjusting the voltages that are applied to the first set of correction electrodes also the voltage applied to the second set has to be changed in order to keep the axial frequency invariant. Specifically, the trap was designed such that it is orthogonal when changing both tuning ratios $T R_{1,2}$ by the same factor. Therefore, during the optimisation configuration for each magnetron radius the correction voltage of both correction electrodes is multiplied by a factor called here " $T R_{\text {comb" }}$ ". In first order, the axial frequency shifts quadratically with the magnetron excitation strength:

$$
\Delta \nu_{z} \approx-\frac{C_{4}}{C_{2}} \frac{3}{2 d^{2}} \nu_{z} \cdot \kappa^{2} \cdot U_{\mathrm{exc}}^{2}+\frac{C_{6}}{C_{2}} \frac{45}{16 d^{4}} \nu_{z} \cdot \kappa^{4} \cdot U_{\mathrm{exc}}^{4}
$$

where $\kappa$ is the magnetron radius calibration factor. Assuming the ion is originally cooled to negligible amplitudes, $\kappa$ relates the magnetron radius to the excitation

\footnotetext{
${ }^{4}$ In an orthogonal trap, the ion's axial frequency is independent of the correction voltages.
} 

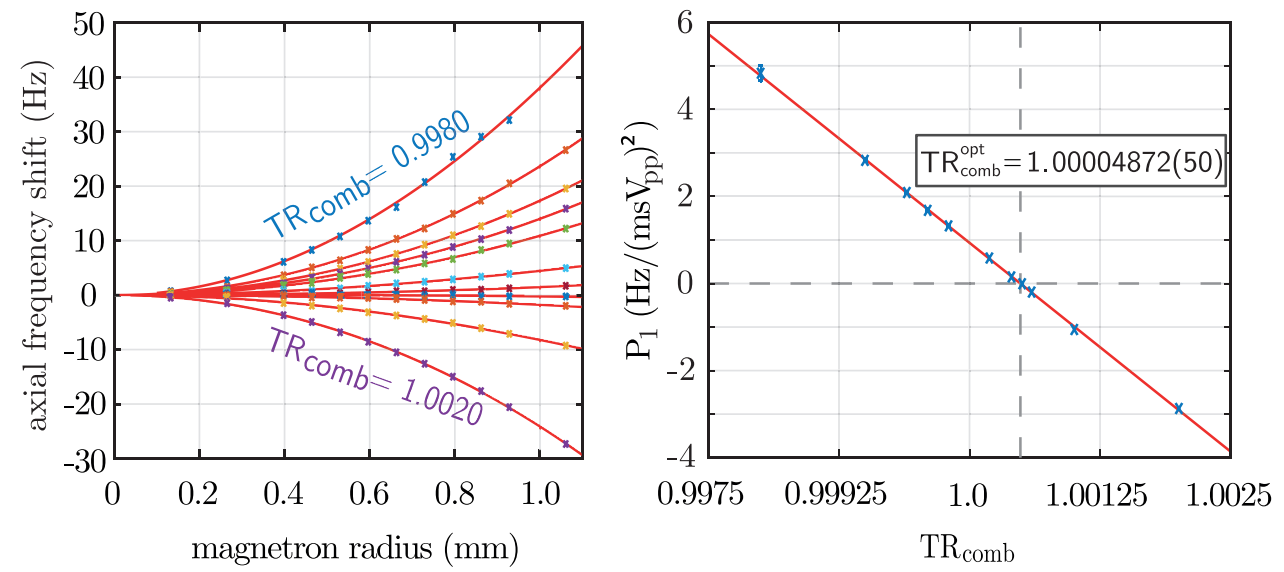

Fig. 31. (Left) Axial frequency shift for different tuning ratios at different radii of the magnetron mode, $\rho_{-}$. The plotted axial frequency shift is the difference between the axial frequency when the magnetron mode is pulsed and thermalised $\left(\nu_{z}^{\text {hot }}-\nu_{z}^{\text {cold }}\right)$. (Right) Polynomial fit parameters $P_{1}\left(\propto C_{4}\right)$ as a function of the combined tuning-ratio factor $T R_{\text {comb. }}$. From the linear fit the optimum value $T R_{\mathrm{comb}}^{\mathrm{opt}}$ is obtained for $P_{1}=0$.

burst as:

$$
\rho_{-}=\kappa \cdot U_{\text {exc }}
$$

With optimal settings, the axial frequency is essentially independent on the oscillation amplitude, demonstrating excellent harmonicity.

From the electrostatic simulation, optimal values of $T R_{1}=0.9636$ and $T R_{2}=$ 0.8156 have been determined. By changing the ions magnetron radius for different $T R_{\text {comb }}$ settings we can map the harmonicity of the trap as can be seen in Figure 31. There, during the tuning-ratio optimization, the strength of the applied excitation was varied via the pulse length whilst the excitation pulse amplitude was fixed to $0.1 \mathrm{~V}_{\mathrm{pp}}$. The obtained data from all individual excitation pulse strength scans for each tuning ratio is fitted using equation (39). We define $P_{1} \equiv-\frac{C_{4}}{C_{2}} \frac{3}{2 d^{2}} \nu_{z} \cdot \kappa^{2}$ and $P_{2} \equiv \frac{C_{6}}{C_{2}} \frac{45}{16 d^{4}} \nu_{z} \cdot \kappa^{4}$. Considering the linear dependence of the $C_{4}$ coefficient with respect to the applied tuning ratio (Fig. 31 (right)), the optimum combined tuning ratio can be obtained. Note that this is in fact the factor that the calculated tuning ratios $T R_{1}$ and $T R_{2}$ need to be multiplied by for a highly harmonic electric field in the centre of the precision trap.

In order to minimise the $C_{4}$ coefficient we chose the tuning ratio factor $T R_{\text {comb }}$ that corresponds to the zero crossing in Figure 31 (right), which is $T R_{\mathrm{comb}}^{\mathrm{opt}}=$ $1.0004872(50)$. This value deviates from the expected calculated value less than one part per thousand which shows a good performance of our experimental setup with respect to the theoretical predictions. From the slope $\alpha$ of the fit in Figure 31 (right) and from equation (39) we obtain the calibration constant $\kappa$ :

$$
\kappa=\sqrt{-\frac{2}{3} \frac{a C_{2} d^{2}}{D_{4}^{\mathrm{comb}} 2 \pi \nu_{z}}}=140(1) \mu \mathrm{m} /(\mathrm{V} \cdot \mathrm{ms}),
$$

where $C_{2}=-0.590325, D_{4}^{\text {comb }}$ is calculated to be $D_{4}^{\text {comb }}=-0.590325, d=$ $9.156470 \mathrm{~mm}$ is the characteristic trap size and $\alpha$ is given by the slope in 


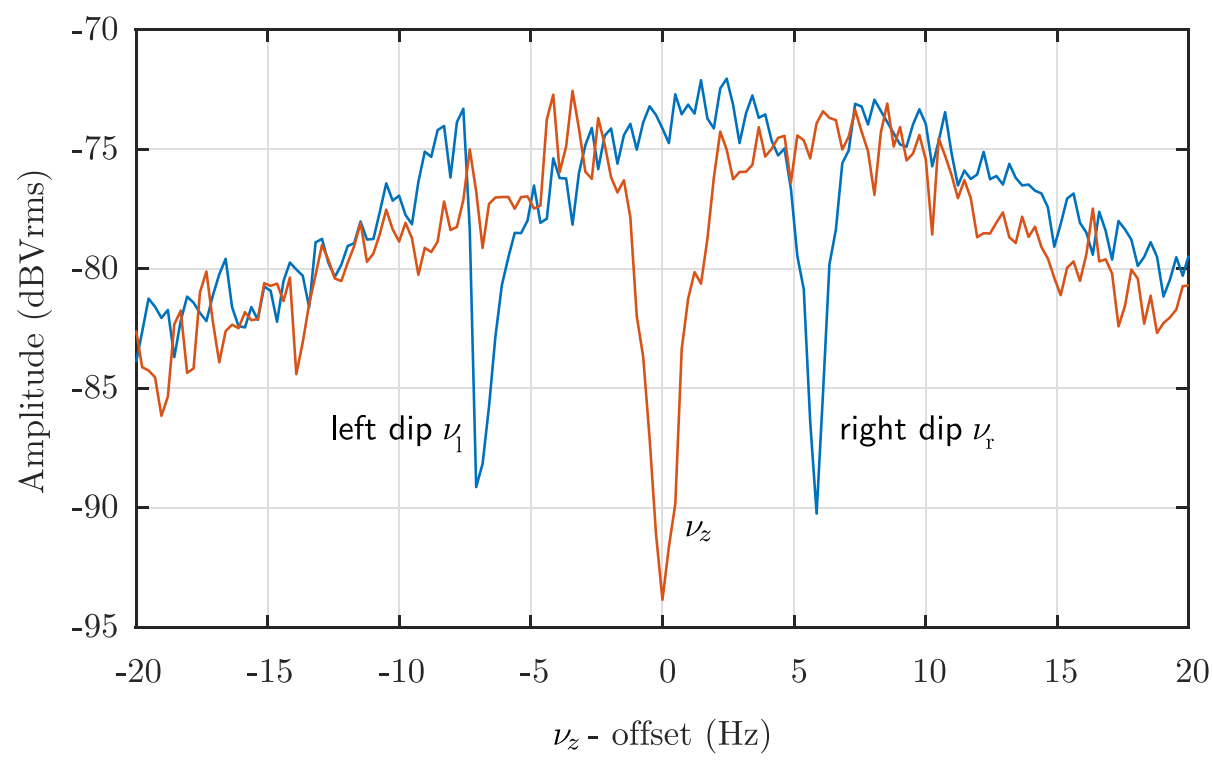

Fig. 32. Sideband coupling at $\nu_{\mathrm{rf}}=\nu_{+}-\nu_{z}$. In red, the typical axial dip with $\nu_{z}$ of the ion when no coupling is applied is shown. In blue the double dip is plotted, with the frequencies of the left $\left(\nu_{1}\right)$ and right $\left(\nu_{\mathrm{r}}\right)$ dips indicated in the figure. The width of each dip of the double dip is half the width of the single axial dip.

Figure 31 (right) as $\alpha=-1917(14) \mathrm{Hz} /\left(\mathrm{msV}_{\mathrm{pp}}\right)^{2}$. The applied excitation pulses of the magnetron mode were dipole rf drives on the $D_{x}$ electrode. It can be observed from Figure 31 (left), that even for large radii up to $1 \mathrm{~mm}$ (compared to the thermal radius of about $1 \mu \mathrm{m}$ ), the axial frequency shifts by significantly less than $1 \mathrm{~Hz}$.

\subsection{Double-dip measurement}

The determination of the $g$-factor requires determining the free cyclotron frequency of the ion (see Sects. 1 and 3), for which three eigenfrequencies need to be measured. The axial mode is measured directly with a detection system that is in resonance with the ion's axial frequency. To measure the other two radial modes, the so called "double-dip technique" is utilised. It is essentially a quadrupole $Q_{x z}$ coupling, as explained in detail in Section 3.4, on the red sideband $\nu_{\mathrm{rf}}=\nu_{+}-\nu_{\mathrm{z}}$. When this rf field is coupled to the ion's motion not only the radial modified cyclotron mode $\nu_{+}$is resistively cooled via the axial detection system, but also $\nu_{+}$can be measured.

Considering the relatively long time constants $(\sim \min )$ of the direct cooling via the cyclotron resonator, the sideband cooling technique via the axial resonator allows for typically much faster cooling, in the order of $100 \mathrm{~ms}$ for HCI.

While the modes are coupled, the energy transfer between the modes occurs with the Rabi frequency $\Omega_{0}$ which depends on the rf amplitude and the detuning. After a few cooling time constants, when both modes are thermalised with the axial resonator, the modified cyclotron frequency can be deduced. In Figure 32 the axial dip and the corresponding double-dip splitting after sideband coupling with $\nu_{\mathrm{rf}}=\nu_{+}-\nu_{\mathrm{z}}$ is shown.

The frequencies of the left and right dips are given by $\nu_{1}=\nu_{z}-\Delta_{0}$ and $\nu_{\mathrm{r}}=\nu_{z}+\Delta_{0}$, respectively. Assuming a non-perfectly resonant coupling rf drive, the 


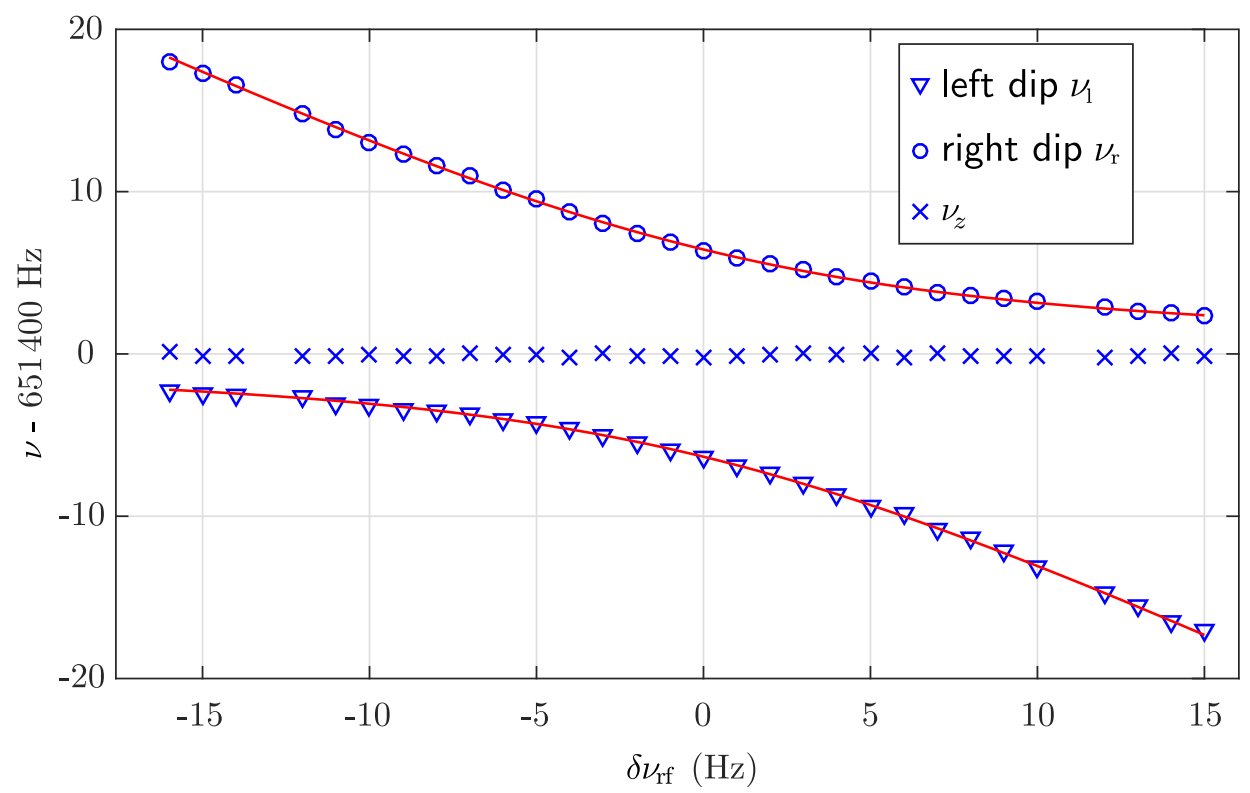

Fig. 33. Avoided crossing of the left and right dips due to coupling of the axial and modified cyclotron modes using the the sideband $\nu_{+}-\nu_{z}$. The axial frequency $\nu_{z}$ is independently measured after each sideband coupling measurement. The fit (red line) is according to equation (42) in [40].

double-dip splitting is no longer symmetric around the axial single dip. Rather, the two dips show an avoided crossing behavior, which can be seen in Figure 33. There, the left, right and non-coupled axial dip are shown. The frequency of each dip is recorded for different coupling frequencies $\nu_{\mathrm{rf}}$ within a frequency range of $\pm 20 \mathrm{~Hz}$.

The avoided crossing data in Figure 33 are fit using the equation given in [40] after a rigorous calculation. For a small detuning $\delta$ around the coupling frequency such that $\nu_{\mathrm{rf}}=\nu_{+}-\nu_{z}+\delta$, the ion oscillates at the axial mode with frequency components

$$
\nu=\nu_{z}-\frac{\delta}{2} \pm \sqrt{\delta^{2}+\Delta_{0}^{2}}
$$

The modified cyclotron frequency can thus be deduced from the relation:

$$
\nu_{+}=\nu_{\mathrm{r}}+\nu_{\mathrm{l}}-\nu_{z}+\nu_{\mathrm{rf}}
$$

which is exact within the rotating wave approximation. Here, the axial frequency $\nu_{z}$ is measured independently when the rf drive is off.

After thermalisation of all modes, the effective temperature of the axial detection circuit can be determined via equation (19). These measurements are discussed in Section 5.6.

\subsection{Determination of $B_{2}$ in the analysis trap}

The strength of the magnetic bottle is determined by measuring the $B_{2}$ coefficient within the trap. According to equation (44), an axial frequency change can 


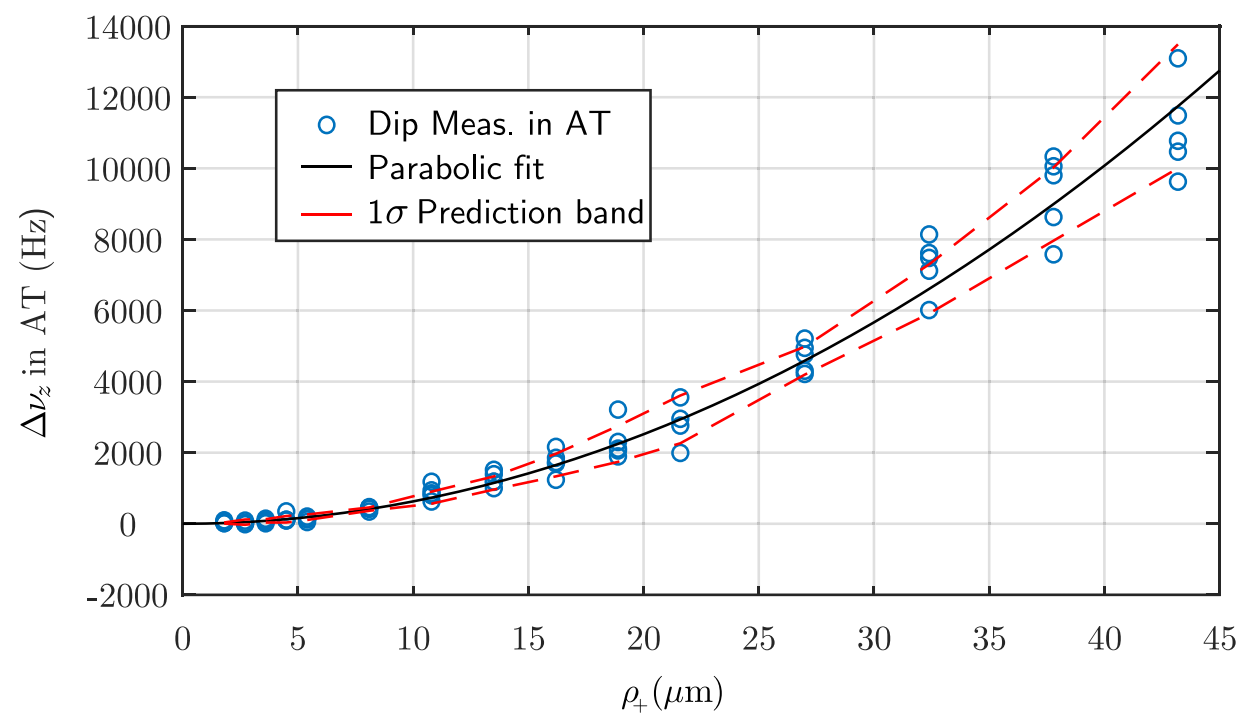

Fig. 34. Strength of the magnetic bottle present in the analysis trap caused by the ferromagnetic ring. The axial frequency shift in the analysis trap is plotted for different modified cyclotron radii of the ion. For details see text.

be observed when the ion's modified cyclotron radius $\rho_{+}$is altered:

$$
\frac{\delta \nu_{z}}{\nu_{z}}=\frac{B_{2}}{4 B_{0}} \frac{\nu_{+}+\nu_{-}}{\nu_{-}} \rho_{+}^{2} .
$$

After having verified that there is no increase of the ion's cyclotron energy during ion transport between precision and analysis trap, the strength of the magnetic bottle in the analysis trap was measured as follows: Initially the cold ion is in the analysis trap where the axial frequency $\nu_{z}$ is measured before the ion is adiabatically transported to the precision trap. There, the modified cyclotron radius $\rho_{+}$of the ion is pulsed to a well-defined value and the ion is transported back to the analysis trap. The magnetic bottle shifts the axial frequency of the ion accordingly and the axial frequency dip of the ion is measured anew. Then the ion is transported once again to the precision trap where the ion's modified cyclotron mode is sideband coupled to the axial mode, therefore the modified cyclotron energy of the previously applied excitation is dumped on the axial resonator and the ion is cooled back via resistive cooling. The modified cyclotron mode radius calibration was obtained independently prior to this measurement and was used here in order to translate the excitation pulse strength into modified cyclotron radius $\rho_{+}$.

The shift of the axial frequency with respect to $\rho_{+}$can be seen in Figure 34 . This measurement allows mapping the strength of the magnetic bottle in the analysis trap. The value that was deduced during this measurement was $B_{2}=44.35(84) \mathrm{kT} / \mathrm{m}^{2}$.

\subsection{Ion transport}

In order to measure the $g$-factor, the ion has to be transported several times from the precision trap to the analysis trap and back. To prevent changes in the spin state or energy of the ion, it is transported adiabatically between a set of electrodes that are located between the two traps. During this process the potential on these 
electrodes is consecutively altered so that the potential trap is "moved" from one electrode to the other. This change has to happen slow enough so that the ion's entropy remains unchanged. This is a restriction mainly for the slow magnetron mode. In order to guarantee a sufficiently high magnetron frequency at any moment of the transport, it is important to have short electrodes, which enables a sufficiently strong quadrupole potential to all positions along the transport path, despite unavoidable patch potentials on the electrodes' surfaces.

In the case of transport between the capture electrodes and the precision trap, the procedure is in principle the same but in that case a cloud of ions is transported before the cloud is reduced down to a single ion (see Sect. 5.1) and resistively cooled. The depth of the trap during transport needs to be sufficient to accommodate the energy distribution of the captured ion bunch within the capture section.

To verify the necessary adiabatic transport of the single ion between the two traps, the ion was transported from the analysis trap where the axial frequency is measured, to the precision trap and immediately back to the analysis trap for a new measurement of the ion's $\nu_{z}$. When the ion arrives in the precision trap, no action is taken that would alter the ion's radial energy, such as sideband coupling. The fluctuation of the axial frequency in the analysis trap, typically $\delta \nu_{z} \approx 0.3 \mathrm{~Hz}$, yields an upper limit for the heating of the modified cyclotron mode by the transport of $1.2 \mathrm{~K}$ for a ${ }^{12} \mathrm{C}^{5+}$ ion.

\subsection{Ion temperature measurement}

Resistive and sideband cooling techniques allow to reduce particle amplitude dependent frequency shifts by thermalising the respective ion mode temperatures with the axial detector circuit. However, the detector circuit can be assigned an effective noise temperature $T_{\text {eff }}$ which, owing to the additional electronic noise contribution of the amplifier, can be much higher than the lattice temperature of the experiment. In this regard ion temperature measurements are an effective way to verify the detector noise performance. As long as the ion and detector frequency are in (or close to) resonance the ion energy will not be constant but fluctuate on timescales of the cooling time constant. According to the ergodic theorem, consecutive measurements of the axial ion energy will reproduce a Boltzmann distribution from which the effective detector/ion temperature $T_{\text {eff }}=T_{z}=\frac{\left\langle E_{z}\right\rangle}{k_{\mathrm{B}}}$ can be extracted. The thermal distribution can be accessed by performing sideband coupling of the axial and the modified cyclotron mode. During sideband coupling the modified cyclotron energy reflects the thermal distribution with the scaled temperature $T_{+}=\frac{\nu_{+}}{\nu_{z}} T_{z}$

$$
\rho\left(E_{+}, T_{+}\right)=\frac{1}{k_{\mathrm{B}} T_{+}} e^{-\frac{E_{+}}{k_{\mathrm{B}} T_{+}}}=\frac{\nu_{z}}{k_{\mathrm{B}} \nu_{+} T_{z}} e^{-\frac{E_{+} \nu_{z}}{k_{\mathrm{B}} \nu T_{z}}} .
$$

This can be easily observed in the strong magnetic bottle of the analysis trap where the modified cyclotron energy is translated into a measurable shift of the axial frequency

$$
\Delta \nu_{z}=\frac{1}{(2 \pi)^{2} m \nu_{z}} \frac{B_{2}}{B_{0}} E_{+}
$$

This way, the thermal distribution can be recorded by measuring the corresponding shifts of the axial frequency after repeated sideband coupling between the axial and the modified cyclotron mode. The result of such a measurement for the axial precision-trap detector is shown in Figure 35. It corresponds to an axial ion/detector 


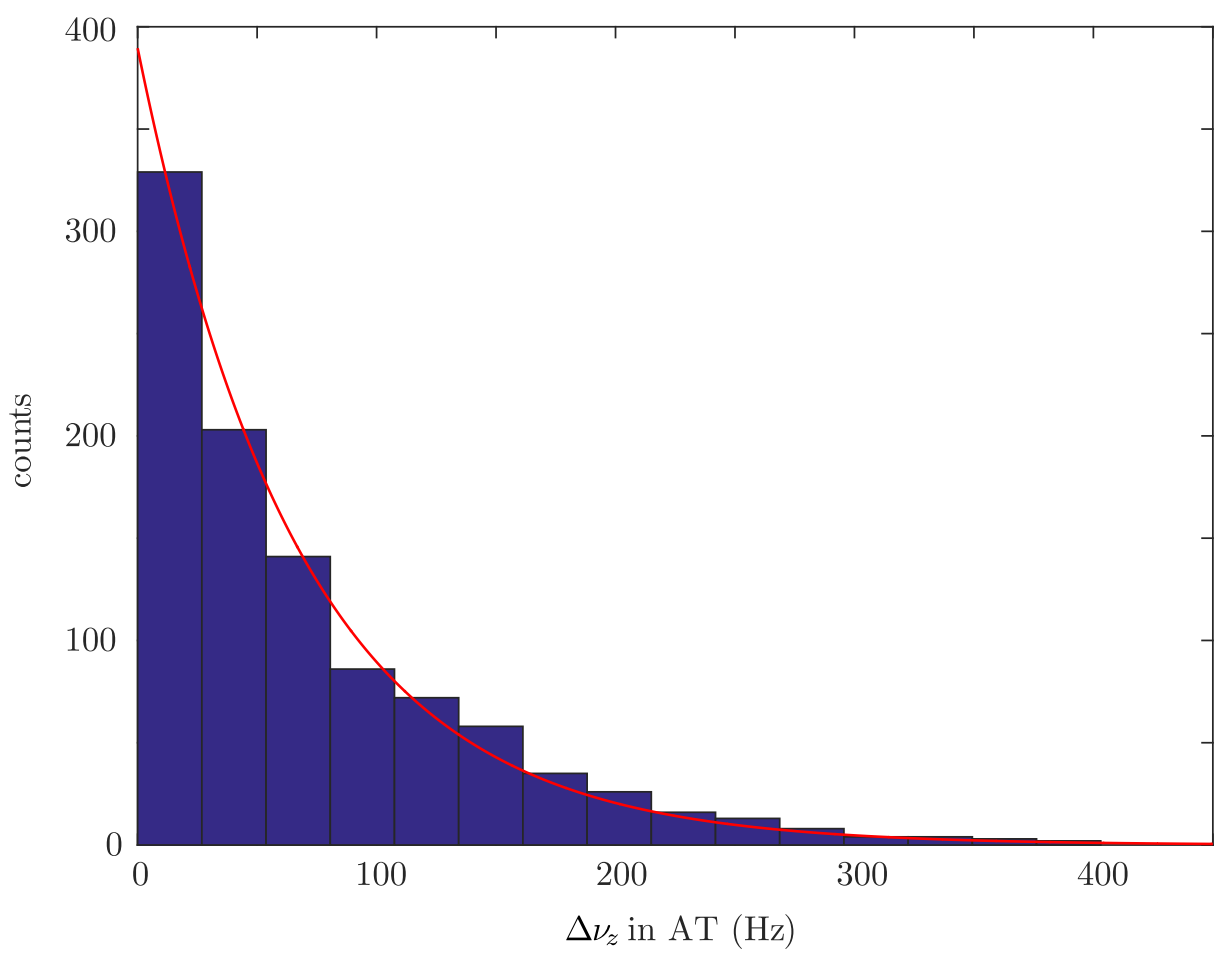

Fig. 35. Histogram plot of the temperature measurement of the axial precision-trap detector. In one measurement cycle the axial and modified cyclotron modes are coupled in the precision trap and transported to the strong magnetic bottle in the analysis trap where the corresponding frequency shift is measured (see text). Here this was repeated for 1000 measurement cycles. The axial frequency shift data reflects the energy distribution of the modified cyclotron/axial mode and allows us to extract the corresponding mode and detector temperatures.

temperature of $T_{z}^{\mathrm{PT}}=12.9(4) \mathrm{K}$. In contrast to the axial analysis-trap detector, the determination of the temperature of the precision-trap detector additionally requires that the modified cyclotron energy is not influenced by the adiabatic transport between the traps which was explicitly verified in Section 5.5.

\subsection{Magnetic field stability}

The significance of a stable magnetic field during our measurement has been already stressed in Section 1. Unlike long-term drifts, the short-term changes in the magnetic field can limit the precision of the measurement. Although the Larmor and cyclotron frequencies are determined simultaneously, fluctuations during the measurement period increase the Larmor resonance linewidth.

AlphatraP's superconducting magnet (see Sect. 4.2) has been shimmed and the resulting magnetic field has been measured with an NMR probe prior to full assembly of the experiment. Afterwards, while measuring the relative stability of $\nu_{+}$the observed frequency drift required the implementation of a self shielding superconducting coil (see Sect. 4.2) which is designed to compensate for externally induced magnetic field gradients by conservation of the flux in the centre of the precision trap. 

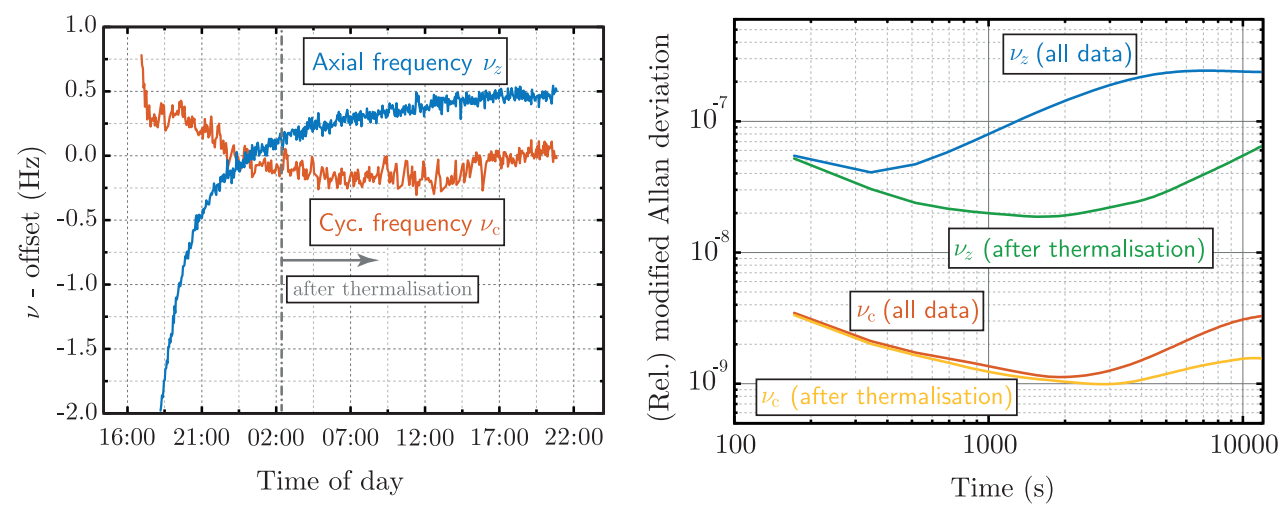

Fig. 36. (Left) Typical fluctuations of the axial and cyclotron frequencies when detected with the dip and double-dip technique. The data was recorded after refilling the cryogen reservoirs and a thermalisation process is clearly visible over the first hours. (Right) Modified Allan deviation of the time sequence on the left. For the green and yellow traces only data after the thermalisation (indicated on the left plot) is used.

The resulting field stability after the superconducting self-shielding coil was installed is demonstrated in Figure 36, where several consecutive measurements of $\nu_{+}$ were taken over the course of about 15 hours. During the same time, the behaviour of the axial frequency is monitored in order to check for stability of the voltage source simultaneously.

The above measurement has been performed by the so-called "double dip" technique (see Sect. 5.3) where via sideband coupling the modified cyclotron frequency can be read out via solely axial detection. Another possibility for magnetic field stability determination, therefore modified cyclotron frequency stability, would be via PnA (see Sect. 3.5). While this technique has not yet been implemented at Alphatrap, the Allan deviation plot suggests a significant improvement possibility, since the fast measurement cycle of $\mathrm{PnA}(\sim 10 \mathrm{~ms})$ gives access to the regime of high stability below 10 min measurement time.

It should be noted that to date, neither the experimental setup nor the voltage source are temperature or pressure stabilised. This is an ongoing project that is expected to improve the frequency stability by reducing the influence of environmental conditions.

\section{Measurement perspectives}

AlPhatrap is a novel and uniquely versatile experiment that enables a whole range of intriguing measurements. The versatility of the available ion sources and detection techniques enables for the first time virtually unrestricted access to all elements, isotopes and charge states for ultra-high precision measurements. Some of the planned experiments at ALPHATRAP are introduced in the following.

\section{1 $g$-Factor measurements in highly charged ions}

The central goal of ALPhATRAP is to test bound-state QED in the strongest fields by comparing the measured $g$-factor of heavy highly charged ions with its prediction by theory. 
The predecessor experiment in Mainz has performed such measurements on light and medium heavy ions, but the miniature EBIT inside the hermetically sealed trap chamber in this experiment limited the choice of ions to those with ionization potentials of less than about $4 \mathrm{keV}$. With its external ion sources, Alphatrap is optimised for the heaviest highly charged ions. The most prominent candidate is the doubly-magic hydrogenlike lead ${ }^{208} \mathrm{~Pb}^{81+}$, which is favorable due to its highlying nuclear excitations and the correspondingly small nuclear contributions on the $g$-factor. The coupling of the electron to the nucleus is characterised by the coupling constant $Z \alpha$. While this coupling is weak $(Z \alpha \ll 1)$ for light and medium heavy ions, for heavy ions $Z \alpha \approx 1$ which brings QED into the highly interesting and up to now largely untested strong coupling regime. The $1 s$ electron in the hydrogenlike lead ${ }^{208} \mathrm{~Pb}^{81+}$ feels an electric field of $\langle E\rangle=1.1 \times 10^{16} \mathrm{~V} / \mathrm{cm}$ (see Fig. 2), which makes a precise $g$-factor measurement on this ion the most stringent test of QED in strong fields.

However, considering the precision achievable in ALPHATRAP, a QED test with ${ }^{208} \mathrm{~Pb}^{81+}$ will be momentarily limited by nuclear contributions [26]. Specifically the nuclear size, which significantly modifies the electrons wave function, has a relative contribution of $2.6 \times 10^{-4}$ to the $g$-factor. Although the charge distribution can be taken from other experiments, the current uncertainty of the charge radius limits the precision of the $g$-factor for the purpose of a QED test to about $4.6 \times 10^{-7}$. To improve the impact of the test further, Shabaev [11] has proposed to consider specific differences of $g$-factors of different charge states. The interaction of the electron with the nucleus is identical for the $1 s$ electron in hydrogenlike lead ${ }^{208} \mathrm{~Pb}^{81+}$ as for the $2 s$ electron in ${ }^{208} \mathrm{~Pb}^{79+}$ up to a well-known scaling factor $\chi$, which can be calculated to high precision. In the specific difference $\Delta g=g_{2 s}-\chi g_{1 s}$ the nuclear contribution are largely canceled, while the electron QED contributions are still present. A similar specific difference can also be constructed from hydrogen and boronlike ions. As AlPhatraP can measure all charge states and isotopes, it provides unique opportunities for QED tests. If QED theory can calculate the $g$-factors of the lithiumlike system to sufficient precision, the specific difference paves the way to previously unattainable sensitivity to new physics.

\subsection{Determination of the electron's atomic mass}

The electron's atomic mass is one of the most important fundamental constants in the Standard Model. Its value, or the ratio of electron and proton mass, is required for virtually every atomic physics precision experiment and the most important tests of the fundamental theories. However, using the standard methods for precision mass measurements, where the cyclotron frequencies of two ions are compared in the same magnetic field, the electron mass is very difficult to measure due to its low mass. Even for low kinetic energies, the relativistic mass increase is sizeable. In the past, our group has successfully demonstrated an alternative approach. Instead of the cyclotron frequency of the electron, we measure the Larmor frequency of a bound electron. As it can be seen in equation (4), this frequency also depends on the electron's mass. If the $g$-factor is known from theory, the magnetic field can be determined by measuring the ion's cyclotron frequency. As the ion is significantly heavier than the bare electron, the cyclotron frequency can be measured with good precision. This way, the electron's mass can be determined in units of the ion's mass. By exploiting the free choice of the nucleus, we choose the ${ }^{12} \mathrm{C}^{5+}$ as the preferred ion for this purpose. This ion not only has a relative weak binding field, such that we can trust in the validity of QED in this regime, its mass (more precisely the mass of the carbon atom) is also the basis of the atomic mass unit and thus does not contribute additional uncertainty apart from the small mass and binding energies of the removed electrons. 
Using this technique, we have published a value of the electron's mass in 2014 [9] with a relative precision of $2.8 \times 10^{-11}$. This is by more than an order of magnitude the most precise value and currently almost exclusively defines the CODATA literature value [89]. In this measurement, the dominant uncertainty is given by the image charge shift, which scales strongly with the trap size. The large ALPHATRAP precision trap, which results in about an order of magnitude lower image charge effect, gives the opportunity to further improve on the published result, if other limitations such as magnetic field stability and ion temperature can be overcome.

\subsection{Detection of long-lived optical transitions via the spin-state detector and laser spectroscopy of highly charged ions}

Beyond $g$-factor measurements, the spin-state detection via the continuous SternGerlach effect opens the possibility for ultra-precise laser spectroscopy in HCI. Due to the strong binding, most electronic transitions in HCI are beyond optical energies. However, depending on the element and charge state, there are specific cases which are accessible to laser spectroscopy. Those transitions can be highly interesting due to their specific properties, as e.g. low susceptibility to external fields and high sensitivity to fundamental constants and interactions. In the medium- $Z$ regime, e.g. for boronlike ${ }^{40} \mathrm{Ar}^{13+}$, the fine structure becomes optical, and at high- $Z$ even the hyperfine transition is laser-accessible. For bismuth, there are even two optical hyperfine transitions, in the hydrogenlike and the lithiumlike state, which enables to make use of a specific difference technique similar as in the $g$-factor case. Since these transitions are M1 transitions, there natural lifetime is rather long, $80 \mathrm{~ms}$ in the case of ${ }^{209} \mathrm{Bi}^{80+}$, which makes a detection of the fluorescence photons rather hard. In Alphatrap, we have the unique opportunity to detect the result of the transition, rather than the transition itself, by driving a transition that pumps the ion into a different spin state (see Fig. 37). In the example of ${ }^{40} \mathrm{Ar}^{13+}$, the ion is prepared by the usual millimeter-wave excitation in the $2 p_{1 / 2}\left(m_{j}=-1 / 2\right)$ state. Then, by driving the $2 p_{1 / 2}\left(m_{j}=-1 / 2\right) \rightarrow 2 p_{3 / 2}\left(m_{j}=+1 / 2\right)$ transition, the ion is eventually left in the dark $2 p_{1 / 2}\left(m_{j}=+1 / 2\right)$ state, which can be observed in the analysis trap by determining the spin state similar as in a $g$-factor measurement. This scheme is virtually insensitive to the lifetime of the excited state. Even if the lifetime is very long, we could detect the spin change directly in the excited state. The Zeeman shift can be calculated using the magnetic field determined by measuring the cyclotron frequency. Typical short-time fluctuations of the magnetic field better than $\delta B / B=10^{-9}$ limit the precision of magnetic field sensitive transitions on the level of $\delta \nu / \nu=10^{-14}$ without further measures. Currently, the main limitation for the achievable precision lies in the first order Doppler shift due to the finite axial temperature, which results in a linewidth of about $158 \mathrm{MHz}$ for ${ }^{40} \mathrm{Ar}^{13+}$ at $4.2 \mathrm{~K}$. In fact, due to the harmonic motion of the trapped ion, the measured spectrum consists of a multitude of individual lines, separated by the axial frequency $(650 \mathrm{kHz})$ and each only about $16 \mathrm{~Hz}$ wide (if we neglect further broadening due to the axial thermalization process and magnetic inhomogeneity). If the spectroscopy laser can resolve this structure, or if the ion can be cooled to lower temperature by means of sympathetic laser cooling, a drastically higher precision can be achieved. If we apply this scheme to the hyperfine structure transitions in ${ }^{209} \mathrm{Bi}^{80+, 82+}$, this would allow for a stringent QED test in strong electric and particularly magnetic fields, which reach about $20000 \mathrm{~T}$ in this system.

\subsection{Sympathetic laser cooling of $\mathrm{HCl}$ and two-ion crystals}

Since laser cooling for free atoms [90] and trapped ions [91] was independently proposed as early as 1975, these techniques are nowadays commonly used to cool ions 


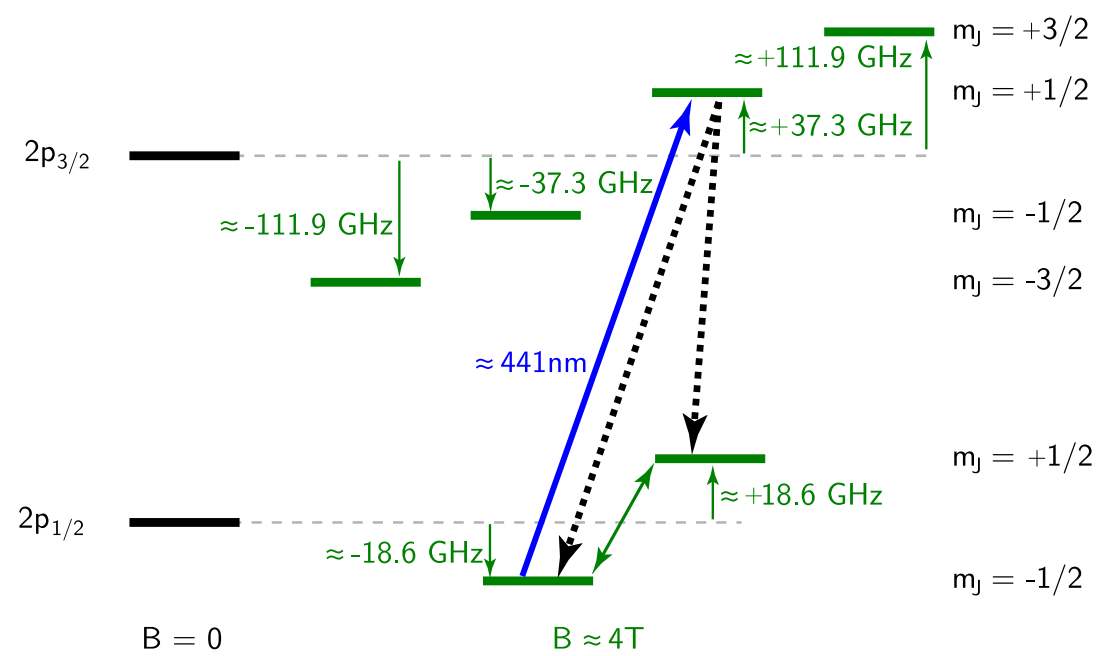

Fig. 37. The level structure of boronlike ${ }^{40} \mathrm{Ar}^{13+}$. The fine structure transition, nominally at about $441 \mathrm{~nm}$, is split by the (non-linear) Zeeman effect. By driving the M1 transition from the spin-down state to the spin-up state in the $2 p_{3 / 2}$ manifold (or from spin up to down), it is possible to pump the ground-state into the spin-up state. This transition can then be detected via the continuous Stern-Gerlach effect, without the need for fluorescence detection. For details see text.

for various purposes such as quantum simulations, quantum information processing and precision laser-spectroscopy e.g. for modern optical clocks. However, even though other Penning trap based $g$-factor experiments are planning to implement laser cooling as well [48], Alphatrap would be, to our knowledge, the first experiment to use these techniques in a Penning trap in the context of high-precision $g$-factor measurements in HCI. Laser cooled ions would improve the envisaged measurement accuracy by lowering some of the systematic, amplitude-dependent frequency-shifts [88], since smaller motional amplitudes of the ion are less prone to imperfections of the trapping fields. Furthermore the second order Doppler shift would be reduced, which potentially enables ultra-precise laser spectroscopy of HCI. The previously mentioned detection methods, such as PnA (Sect. 3.5), for measuring the ions motional frequencies are at some point limited in the achievable precision by the initial temperature of the ion. For the spin flip analysis of heavy highly charged ions, the fidelity would benefit from an improved stability of the cyclotron energy, since the axial frequency difference $\Delta \nu_{z}$ (Eq. (25)) scales with $\frac{1}{\sqrt{m_{\text {ion }}}}$. As shown in Section 4.5.3, the current axial stability in the analysis trap seems sufficient for resolving spin flips for light hydrogenlike ions in our setup. This small frequency difference even decreases to about one third when measuring ions with a boronlike electron configurations (see Tab. 2). In the strong magnetic bottle of the analysis trap, also the axial frequency will depend on the motional energy in the radial modes [88]. Fluctuations in this energy exacerbates an unambiguous determination of a spin flip. In each measurement cycle the ion is transported from the precision trap to the analysis trap and arrives there with a certain energy in the modified cyclotron mode $E_{+}$chosen from a temperature equivalent thermal Boltzmann distribution. In other experiments $[47,92]$ the dependence of the frequency stability on $E_{+}$was shown. Hence a lower cyclotron temperature and therefore a more narrow Boltzmann distribution of these energies would significantly facilitate the detection.

Even though both sympathetic laser cooling of ions of two different species $[49,93]$ and Coulomb crystallization of ions in a Penning trap has been shown before, we 
plan to extend these methods to a two-ion crystal consisting of a ${ }^{9} \mathrm{Be}^{+}$and an highly charged ion. Other experiments showed that Coulomb crystallization with as few as two (same species) ions in an axial configuration is feasible [94]. In this kind of configuration for the axial velocity components of the two ions two normal modes can be distinguished: a "breathing mode" in which the two ions oscillate symmetrically around the non-moving common center of mass and hereby changing their inter ion separation distance, or a "common mode" motion in which the center of mass can oscillate. The axial inter ion separation $\Delta z$ depends on the axial confinement resp. the potential well depth and amount in our setup typically to around a few ten microns for a ${ }^{9} \mathrm{Be}^{+}$and a HCI. Due to this dependency on the potential well depth, $\Delta z$ can be predicted and well controlled. Having these two ions confined in such a small distance to each other does significantly decrease the differential magnetic field differences seen by the two ions, which enables ultra-precise determinations of $g$-factor differences as outlined in Section 6.5.

\subsection{Ultra-precise determination of $g$-factor differences and the fine-structure constant}

The electron $g$-factor measurements discussed above are performed as Rabi-type spectroscopy. In principle, the Ramsey technique would be much better suited for our experiments, as the coherent Ramsey measurement probes the phase of the Larmor precession

$$
\varphi_{\mathrm{L}, i}(T)=\frac{g_{i}}{2} \frac{e}{m_{e}} \int_{0}^{T} B(t) d t=\left\langle\omega_{\mathrm{L}, i}\right\rangle T,
$$

which depends solely on the mean magnetic field $\langle B\rangle=\frac{1}{T} \int_{0}^{T} B(t) d t$ during the measurement, just as the phase $\varphi_{+}$of the cyclotron frequency. This value is exactly accessible via a PnA measurement, so that in the ratio of the two frequencies measured in this way the magnetic field jitter completely drops out, while in the Rabi-type measurement the jitter causes significant line broadening. However, in practice, the application of the Ramsey method for an electron spin resonance experiment is prohibited by the large ratio of the Larmor and cyclotron frequencies. In order to perform a measurement of the cyclotron frequency to about $10^{-10}$, even with the phasesensitive PnA method, we require about $10 \mathrm{~s}$ measurement time. Within this time, the typical magnetic field fluctuations cause a jitter of $\varphi_{\mathrm{L}}(10 s)$ of significantly more than $360^{\circ}$. In general, to preserve coherence, a phase resolution of the cyclotron motion of $\delta \varphi_{+}<\nu_{+} / \nu_{\mathrm{L}} \cdot 360^{\circ}=0.08^{\circ}$ would be required, which is currently inaccessible.

However, if we consider two different ions in the same charge state, their respective Larmor phases $\varphi_{\mathrm{L}, i}(T)$ vary only slowly with respect to each other, because $g_{i}$ depends only weakly on the specific element or isotope. As an example, even for ${ }^{206,208} \mathrm{~Pb}^{81+}$, the isotope shift of the $g$-factor amounts to only $2.7 \times 10^{-7}$ absolute [95]. Consequently, if we could directly measure the small difference of these two phases

$$
\Delta \varphi_{\mathrm{L}}(T)=\frac{g_{2}-g_{1}}{2} \frac{e}{m_{e}} \int_{0}^{T} B(t) d t,
$$

this measurement would easily stay coherent. This becomes possible by placing two ions in the same magnetic field, either in two close-by traps, or ideally with the help of sympathetic laser cooling in form of an axial Coulomb crystal in one trap (see Sect. 6.4 and Fig. 38). The key idea is to perform independent, but simultaneous 


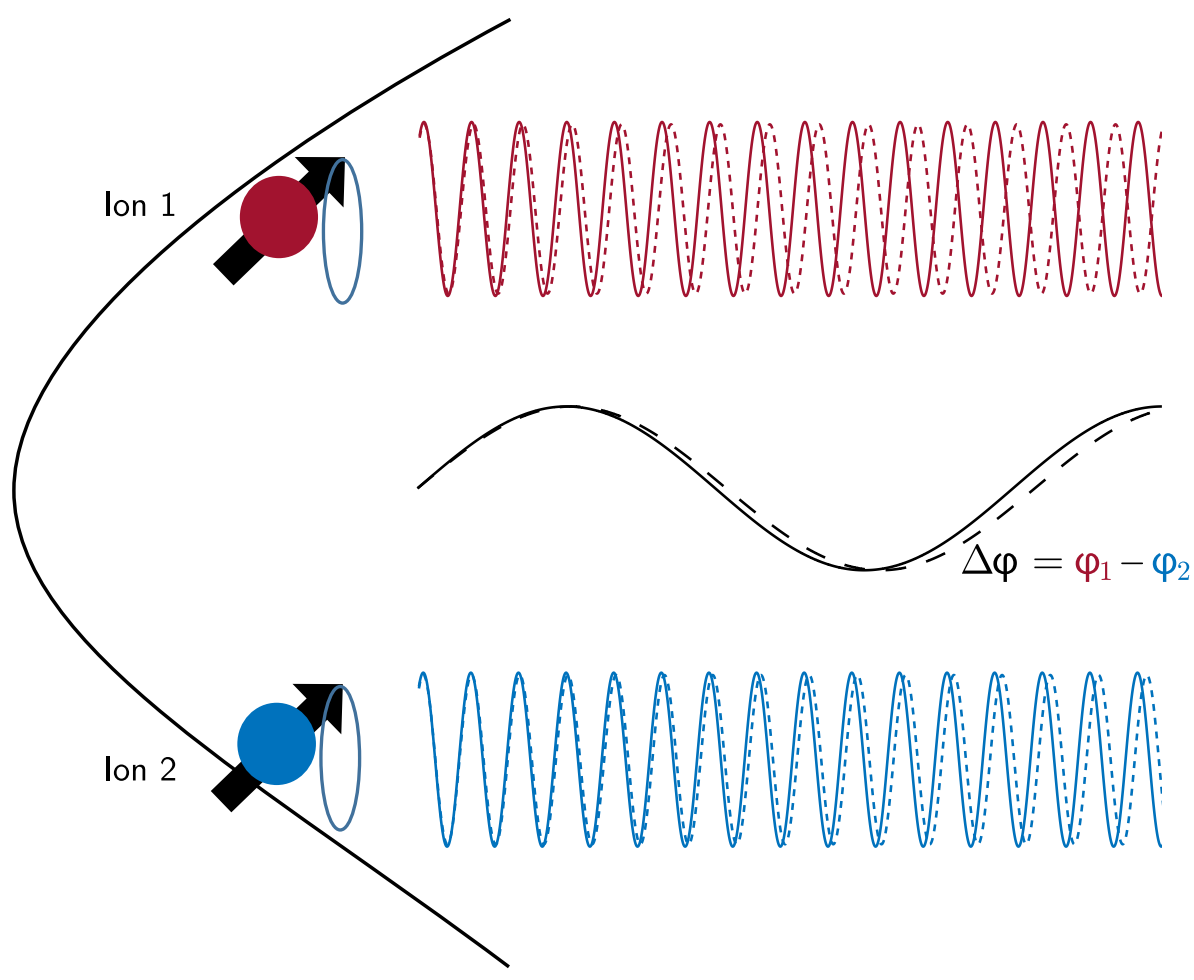

Fig. 38. Sketch of the individual phase evolutions of the two co-trapped ions and their difference for two generic magnetic field fluctuations, indicated as the solid and dashed lines, respectively. While the individual Larmor precession loses coherence during the measurement period, the difference stays measurable and allows sensitive access to differential ion properties.

Ramsey measurements on these ions, and detect the individual spin-states one after another in the analysis trap. This way, the probability to find e.g. exactly the first ion in spin-state "up" (assuming w.l.o.g. both ions are prepared in spin "down" originally) is

$$
\begin{aligned}
p(|\uparrow, \downarrow\rangle) & =p_{1}(|\uparrow\rangle) p_{2}(|\downarrow\rangle) \\
& =\cos \left(\left(\omega_{L, 1}-\omega_{\text {drive }}\right) T\right)^{2} \sin \left(\left(\omega_{L, 2}-\omega_{\text {drive }}\right) T\right)^{2} \\
& =\frac{1}{4}\left(\sin \left(\left(\omega_{L, 1}-\omega_{L, 2}\right) T\right)+\ldots\right)
\end{aligned}
$$

where terms varying with high frequencies are neglected. While these high frequency terms are smeared out by the magnetic field fluctuations, the slow terms stay visible. An easily reachable $1^{\circ}$ resolution with 200 s measurement time thus results 16 digits sensitivity with respect to the $g$-factor. Consequently, the sensitivity for the $g$-factor difference, e.g. the isotopic shift, can be as large as 11 digits, depending on the magnitude of the difference and provided that differential magnetic field fluctuations between the two ions can be neglected. This unique resolution enables a whole new range of intriguing experiments, which search for tiny differential contributions, while cancelling large fractions of the "uninteresting", known QED. One immediate application would be the determination of the fine-structure constant $\alpha$ from the difference of $g$-factors of light ions. Contrary to the free electron $g-2$ experiment, our 
technique does depend in first order on the relativistic contribution (which cancel in the difference), rather than QED: $g=\frac{2}{3}\left(1+2 \sqrt{1-Z \alpha^{2}}\right)$, so that it could potentially allow for an independent determination of $\alpha$.

\section{Conclusion and outlook}

The Alphatrap experiment is a next-generation setup aiming to probe the boundaries of validity of quantum electrodynamics and the Standard Model of Physics in the strongest fields. To this end, the design, which is based on the $g$-factor experiment on highly charged ions at the Johannes Gutenberg-University in Mainz, Germany, has been upgraded with novel techniques to allow ultra-high precision experiments with heavy HCI. In Alphatrap, ions can be injected from a selection of external ion sources, among those the Heidelberg EBIT, which can eventually provide HCI up to hydrogenlike lead ${ }^{208} \mathrm{~Pb}^{81+}$. Furthermore, the new setup allows for sympathetic laser cooling of the HCI as well as performing laser spectroscopy of highly forbidden, narrow transitions, which are difficult or impossible to address in other experiments. This way, ALPHATRAP will open up a new field for intriguing, stringent tests of QED and for the determination of fundamental constants. In the first runs of ALPHATRAP, which are described in this article, basic techniques such as the injection and capturing of clouds of HCI from external sources and the detection of single ions have been demonstrated. The novel, highly harmonic trap has been commissioned and shows excellent performance. For the first time, a cryogenically operable vacuum valve has been successfully implemented, which allows achieving outstanding vacuum conditions in excess of $10^{-17} \mathrm{mbar}$, allowing the virtually unlimited storage even of heavy HCI. Recently, the first determination of the spin-state of a boronlike ${ }^{40} \mathrm{Ar}^{13+}$ ion was demonstrated, which paves the way to the first $g$-factor measurements in ALPHATRAP. In the near future, ALPHATRAP will perform intriguing experiments in a previously unexplored regime and shed light on physics in the strongest electromagnetic fields.

Open access funding provided by Max Planck Society. We acknowledge financial support by the Max-Planck Society. B.T. was supported by a Humboldt Research Fellowship for Postdoctoral Researchers, A.W, I.A. and T.S. were supported by the International MaxPlanck Research School on Quantum Dynamics (IMPRS-QD). This work is part of and supported by the German Research Foundation (DFG) Collaborative Research Centre "SFB 1225 (ISOQUANT)".

Open Access This is an open access article distributed under the terms of the Creative Commons Attribution License (http://creativecommons.org/licenses/by/4.0), which permits unrestricted use, distribution, and reproduction in any medium, provided the original work is properly cited.

\section{References}

1. D. Hanneke, S. Fogwell, G. Gabrielse, Phys. Rev. Lett. 100, 120801 (2008)

2. L. Windholz, E. Winklhofer, R. Drozdowski, J. Kwela, T. Waşowicz, J. Heldt, Phys. Scr. 78, 065303 (2008)

3. T. Stöhlker, P.H. Mokler, K. Beckert, F. Bosch, H. Eickhoff, B. Franzke, M. Jung, Y. Kandler, O. Klepper, C. Kozhuharov et al., Phys. Rev. Lett. 71, 2184 (1993)

4. M. Kozlov, M. Safronova, J. López-Urrutia, P. Schmidt, arXiv:1803.06532 (2018)

5. A. Gumberidze, T. Stöhlker, D. Banaś, K. Beckert, P. Beller, H. Beyer, F. Bosch, S. Hagmann, C. Kozhuharov, D. Liesen et al., Phys. Rev. Lett. 94, 223001 (2005) 
6. S. Sturm, A. Wagner, B. Schabinger, J. Zatorski, Z. Harman, W. Quint, G. Werth, C.H. Keitel, K. Blaum, Phys. Rev. Lett. 107, 023002 (2011)

7. S. Sturm, A. Wagner, M. Kretzschmar, W. Quint, G. Werth, K. Blaum, Phys. Rev. A 87, 030501 (2013)

8. F. Köhler, K. Blaum, M. Block, S. Chenmarev, S. Eliseev, D.A. Glazov, M. Goncharov, J. Hou, A. Kracke, D.A. Nesterenko et al., Nat. Commun. 7, 10246 (2016)

9. S. Sturm, F. Köhler, J. Zatorski, A. Wagner, Z. Harman, G. Werth, W. Quint, C.H. Keitel, K. Blaum, Nature 506, 467 (2014)

10. J.C. López-Urrutia, J. Braun, G. Brenner, H. Bruhns, C. Dimopoulou, I. Draganić, D. Fischer, A.G. Martínez, A. Lapierre, V. Mironov et al., J. Phys.: Conf. Ser. 2, 42 (2004)

11. V. Shabaev, D. Glazov, N. Oreshkina, A. Volotka, G. Plunien, H.J. Kluge, W. Quint, Phys. Rev. Lett. 96, 253002 (2006)

12. J. Vollbrecht, Z. Andelkovic, A. Dax, W. Geithner, C. Geppert, C. Gorges, M. Hammen, V. Hannen, S. Kaufmann, K. König et al., J. Phys.: Conf. Ser. 583, 012002 (2015)

13. J. Ullmann, Z. Andelkovic, C. Brandau, A. Dax, W. Geithner, C. Geppert, C. Gorges, M. Hammen, V. Hannen, S. Kaufmann et al., Nat. Commun. 8, 15484 (2017)

14. L.V. Skripnikov, S. Schmidt, J. Ullmann, C. Geppert, F. Kraus, B. Kresse, W. Nörtershäuser, A.F. Privalov, B. Scheibe, V.M. Shabaev et al., Phys. Rev. Lett. 120, 093001 (2018)

15. W. Gerlach, O. Stern, Z. Phys. 9, 349 (1922)

16. G.E. Uhlenbeck, S. Goudsmit, Naturwissenschaften 13, 953 (1925)

17. P.A.M. Dirac, Proc. R. Soc. Lond. A 117, 610 (1928)

18. P. Kusch, H. Foley, Phys. Rev. 72, 1256 (1947)

19. J. Schwinger, Phys. Rev. 73, 416 (1948)

20. T. Aoyama, M. Hayakawa, T. Kinoshita, M. Nio, Phys. Rev. Lett. 109, 111807 (2012)

21. S. Laporta, Phys. Lett. B 772, 232 (2017)

22. R.H. Parker, C. Yu, W. Zhong, B. Estey, H. Müller, Science 360, 191 (2018)

23. G. Breit, Nature 122, 649 (1928)

24. Z. Harman, private communication, 2016

25. W. Furry, Phys. Rev. 81, 115 (1951)

26. S.G. Karshenboim, V.G. Ivanov, Phys. Rev. A 97, 022506 (2018)

27. A.V. Volotka, G. Plunien, Phys. Rev. Lett. 113, 023002 (2014)

28. V. Shabaev, V. Yerokhin, Phys. Rev. Lett. 88, 091801 (2002)

29. A. Volotka, D. Glazov, V. Shabaev, I. Tupitsyn, G. Plunien, Phys. Rev. Lett. 112, $253004(2014)$

30. A. Varentsova, V. Agababaev, D. Glazov, A. Volchkova, A. Volotka, V. Shabaev, G. Plunien, Phys. Rev. A 97, 043402 (2018)

31. H. Dehmelt, Proc. Natl. Acad. Sci. USA 83, 2291 (1986)

32. K. Pachucki, A. Czarnecki, U.D. Jentschura, V.A. Yerokhin, Phys. Rev. A 72, 022108 (2005)

33. A. Czarnecki, R. Szafron, Phys. Rev. A 94, 060501 (2016)

34. F.T. Köhler, Ph.D. thesis, Ruprecht-Karls-Universität Heidelberg, 2015

35. G. Gabrielse, F.C. MacKintosh, Int. J. Mass Spectr. Ion Process. 57, 1 (1984)

36. L.S. Brown, G. Gabrielse, Rev. Modern Phys. 58, 233 (1986)

37. G. Gabrielse, Phys. Rev. Lett. 102, 172501 (2009)

38. X. Feng, M. Charlton, M. Holzscheiter, R. Lewis, Y. Yamazaki, J. Appl. Phys. 79, 8 (1996)

39. G.J. Ketter, Ph.D. thesis, Ruprecht-Karls-Universität Heidelberg, 2015

40. E.A. Cornell, R.M. Weisskoff, K.R. Boyce, D.E. Pritchard, Phys. Rev. A 41, 312 (1990)

41. F. Heiße, F. Köhler-Langes, S. Rau, J. Hou, S. Junck, A. Kracke, A. Mooser, W. Quint, S. Ulmer, G. Werth et al., Phys. Rev. Lett. 119, 033001 (2017)

42. S. Sturm, A. Wagner, B. Schabinger, K. Blaum, Phys. Rev. Lett. 107, 143003 (2011)

43. VACUUMSCHMELZE, VACOFLUX50, VACUUMSCHMELZE GmbH, Grüner Weg 37, D-63450 Hanau, Germany, 2017, https://www.vacuumschmelze.com/en/products/ materials-parts/soft-magnetic/cobalt-iron/vacoflux/vacoflux-magnetic-properties.html 
44. W. Quint, D. Moskovkhin, V. Shabaev, M. Vogel, Phys. Rev. A 78, 032517 (2008)

45. H. Nagahama, C. Smorra, S. Sellner, J. Harrington, T. Higuchi, M. Borchert, T. Tanaka, M. Besirli, A. Mooser, G. Schneider et al., Nat. Commun. 8, 14084 (2017)

46. H. Häffner, T. Beier, S. Djekić, N. Hermanspahn, H.J. Kluge, W. Quint, S. Stahl, J. Verdú, T. Valenzuela, G. Werth, Eur. Phys. J. D 22, 163 (2003)

47. A. Mooser, Ph.D. thesis, Johannes Gutenberg-Universität in Mainz, 2013

48. M. Bohman, A. Mooser, G. Schneider, N. Schön, M. Wiesinger, J. Harrington, T. Higuchi, H. Nagahama, C. Smorra, S. Sellner et al., J. Modern Opt. 65, 568 (2018)

49. D.J. Larson, J.C. Bergquist, J.J. Bollinger, W.M. Itano, D.J. Wineland, Phys. Rev. Lett. 57, 70 (1986)

50. T. Andersen, K.A. Jessen, G. Sørensen, Phys. Rev. 188, 76 (1969)

51. S. Sturm, Ph.D. thesis, Johannes Gutenberg-Universität in Mainz, 2011

52. W.M. Itano, D.J. Wineland, Phys. Rev. A 25, 35 (1982)

53. D.J. Wineland, W.M. Itano, J.C. Bergquist, R.G. Hulet, Phys. Rev. A 36, 2220 (1987)

54. S. Mavadia, G. Stutter, J. Goodwin, D. Crick, R. Thompson, D. Segal, Phys. Rev. A 89, $032502(2014)$

55. F. Diedrich, J. Bergquist, W.M. Itano, D. Wineland, Phys. Rev. Lett. 62, 403 (1989)

56. J.J. Bollinger, J.S. Wells, D.J. Wineland, W.M. Itano, Phys. Rev. A 31, 2711 (1985)

57. S. Kraemer, Master's thesis, Ruprecht-Karls-Universität Heidelberg, 2017

58. T. Beier, L. Dahl, H.J. Kluge, C. Kozhuharov, W. Quint, H. collaboration et al., Nucl. Instr. Methods Phys. Res. Sect. B 235, 473 (2005)

59. P. Micke, S. Kühn, L. Buchauer, J. Harries, T.M. Bücking, K. Blaum, A. Cieluch, A. Egl, D. Hollain, S. Kraemer et al., Rev. Sci. Instr. 89, 063109 (2018)

60. J. Repp, C. Böhm, J.C. López-Urrutia, A. Dörr, S. Eliseev, S. George, M. Goncharov, Y.N. Novikov, C. Roux, S. Sturm et al., Appl. Phys. B 107, 983 (2012)

61. C. Roux, C. Böhm, A. Dörr, S. Eliseev, S. George, M. Goncharov, Y.N. Novikov, J. Repp, S. Sturm, S. Ulmer et al., Appl. Phys. B 107, 997 (2012)

62. C. Carlberg, H. Borgenstrand, G. Rouleau, R. Schuch, F. Söderberg, I. Bergström, R. Jertz, T. Schwarz, J. Stein, G. Bollen et al., Phys. Scr. 1995, 196 (1995)

63. G. Gabrielse, J. Tan, J. Appl. Phys. 63, 5143 (1988)

64. A. Rischka, Bachelor's thesis, Ruprecht-Karls-Universität, Heidelberg, 2011

65. A. Mooser, S. Ulmer, K. Blaum, K. Franke, H. Kracke, C. Leiteritz, W. Quint, C.C. Rodegheri, C. Smorra, J. Walz, Nature 509, 596 (2014)

66. S. Ulmer, C. Smorra, A. Mooser, K. Franke, H. Nagahama, G. Schneider, T. Higuchi, S. Van Gorp, K. Blaum, Y. Matsuda et al., Nature 524, 196 (2015)

67. C. Smorra, S. Sellner, M. Borchert, J. Harrington, T. Higuchi, H. Nagahama, T. Tanaka, A. Mooser, G. Schneider, M. Bohman et al., Nature 550, 371 (2017)

68. J. Alonso, K. Blaum, S. Djekic, H.J. Kluge, W. Quint, B. Schabinger, S. Stahl, J. Verdú, M. Vogel, G. Werth, Rev. Sci. Instr. 77, 03A901 (2006)

69. T. Sailer, Master's thesis, Ruprecht-Karls-Universität Heidelberg, 2017

70. W.C. Wiley, I.H. McLaren, Rev. Sci. Instr. 26, 1150 (1955)

71. Ensinger, TECAPEEK ELS nanoblack, Ensinger GmbH, Rudolf-Diesel Str. 8, 71154 Nufringen, Germany, version ac edn. (2017), https://www.ensingerplastics.com/en/ shapes/products/peek-tecapeek-els-nano-black

72. M. Schuh et al., in preparation (2018)

73. F. Heiße et al., in preparation (2018)

74. A. Sikdar, A. Ray, P. Das, A. Reza, Nucl. Instr. Methods Phys. Res. Sect. A 712, 174 (2013)

75. K. Farrar, Nucl. Instr. Methods Phys. Res. Sect. A 485, 780 (2002)

76. UM - Series, Multichannel Precision Voltage Source, Stahl-Electronics, Kellerweg 23, 67582 Mettenheim, Germany, manual_um_ln_sw_v2016 edn., 2016, http://www. stahl-electronics.com/bilder/Manual_UM_LN_SW_V2016.pdf

77. A. Weigel, Master's thesis, Ruprecht-Karls-Universität Heidelberg, 2014

78. D. Pavičić, K. Maglić, Int. J. Thermophys. 23, 1319 (2002)

79. S. Ulmer, Ph.D. thesis, Ruprecht-Karls-Universität Heidelberg, 2011 
80. C. Böhm, S. Sturm, A. Rischka, A. Dörr, S. Eliseev, M. Goncharov, M. Höcker, J. Ketter, F. Köhler, D. Marschall et al., Nucl. Instr. Methods Phys. Res. Sect. A 828, 125 (2016)

81. T. Sailer, Bachelor's thesis, Ruprecht-Karls-Universität Heidelberg, 2015

82. T. Steinsberger, Master's thesis, Ruprecht-Karls-Universität Heidelberg, 2018

83. Toptica Photonics AG, Scientific lasers, Brochure br-199-54a, 2015

84. HighFinesse, Wavelength Meter Ångstrom WS Ultimate 2, HighFinesse GmbH, Laser and Electronic Systems, Auf der Morgenstelle 14D, 72076 Tübingen, Germany (n.d.)

85. Lasertex, Iodine stabilised HeNe laser, Model: LJSC-3-11, Lasertex Co. Ltd., 51-501 Wrocław, ul. Swojczycka 26, Poland (n.d.)

86. L. Ricci, M. Weidemüller, T. Esslinger, A. Hemmerich, C. Zimmermann, V. Vuletic, W. König, T.W. Hänsch, Opt. Commun. 117, 541 (1995)

87. T. Photonics, DL-/TA-SHG/FHG pro, Toptica Photonics AG, Lochhamer Schlag 19, 82166 Graefling/München, m-078 version 01 edn., 2016

88. J. Ketter, T. Eronen, M. Höcker, S. Streubel, K. Blaum, Int. J. Mass Spectr. 358, 1 (2014)

89. P.J. Mohr, Rev. Mod. Phys. 88, 035009 (2016)

90. T. Hänsch, A. Schawlow, Opt. Commun. 13, 68 (1975)

91. D. Wineland, H. Dehmelt, Bull. Am. Phys. Soc. 20, 637 (1975)

92. A. Mooser, H. Kracke, K. Blaum, S.A. Bräuninger, K. Franke, C. Leiteritz, W. Quint, C.C. Rodegheri, S. Ulmer, J. Walz, Phys. Rev. Lett. 110, 140405 (2013)

93. L. Gruber, J.P. Holder, J. Steiger, B.R. Beck, H.E. DeWitt, J. Glassman, J.W. McDonald, D.A. Church, D. Schneider, Phys. Rev. Lett. 86, 636 (2001)

94. D.R. Crick, H. Ohadi, I. Bhatti, R.C. Thompson, D.M. Segal, Opt. Express 16, 2351 (2008)

95. V. Shabaev, D. Glazov, M. Shabaeva, V. Yerokhin, G. Plunien, G. Soff, Phys. Rev. A 65, $062104(2002)$ 